\title{
Illustrating Appalachia: The sketchbooks and publications of David Hunter Strother, 1833 to 1887
}

\author{
Alee Robins
}

Follow this and additional works at: https://researchrepository.wvu.edu/etd

\section{Recommended Citation}

Robins, Alee, "Illustrating Appalachia: The sketchbooks and publications of David Hunter Strother, 1833 to 1887" (2015). Graduate Theses, Dissertations, and Problem Reports. 6515.

https://researchrepository.wvu.edu/etd/6515

This Thesis is protected by copyright and/or related rights. It has been brought to you by the The Research Repository @ WVU with permission from the rights-holder(s). You are free to use this Thesis in any way that is permitted by the copyright and related rights legislation that applies to your use. For other uses you must obtain permission from the rights-holder(s) directly, unless additional rights are indicated by a Creative Commons license in the record and/ or on the work itself. This Thesis has been accepted for inclusion in WVU Graduate Theses, Dissertations, and Problem Reports collection by an authorized administrator of The Research Repository @ WVU. For more information, please contact researchrepository@mail.wvu.edu. 


\title{
ILLUSTRATING APPALACHIA: THE SKETCHBOOKS AND PUBLICATIONS OF DAVID HUNTER STROTHER, 1833 TO 1887
}

\author{
by \\ Alee Robins \\ Thesis submitted to the Eberly College of Arts and Sciences \\ at West Virginia University \\ in partial fulfillment of the requirements \\ for the degree of \\ Master of Arts \\ in \\ History \\ Approved by \\ Kenneth Fones-Wolf, Ph.D., Committee Chairperson \\ Melissa Bingmann, Ph.D. \\ Rhonda Reymond, Ph.D. \\ Department of History \\ Morgantown, West Virginia \\ 2015 \\ Keywords: David Hunter Strother, Porte Crayon, Illustrators, Appalachia, \\ Canaan Valley, Blackwater Falls,
}

Copyright 2015 Alee Robins 


\section{ABSTRACT \\ Illustrating Appalachia: The Sketchbooks and Publications of David Hunter Strother, 1833 to 1887}

\section{by Alee Robins}

In the mid-nineteenth century, one of the most popular American magazine illustrators was David Hunter Strother, a native of Martinsburg, Virginia (now West Virginia). Using the pseudonym "Porte Crayon," Strother published semi-fictional accounts of his travels which he often illustrated as well as authored. The West Virginia \& Regional History Center offers a digital collection of 730 of the artist's sketches and paintings. Additionally, the Walters Art Museum presents eight of Strother's sketches online. To provide a method for studying Strother's artworks, a database was created to show connections between the artist's works based on date, location, and thematic content. To show the utility of this database, Strother's sketches from his 1852 trip to the Canaan Valley, in West Virginia, along with his published article about the trip were analyzed. Strother's stories were among the earliest and most wide-spread depictions of Appalachia, making him an influential force in informing the nation about the region. Using an interdisciplinary approach, a study of his images of the Canaan Valley revealed how he characterized the region as a beautiful but rugged wilderness whose inhabitants were skilled but simple outdoorsmen. 
Table of Contents

ABSTRACT ...............................................................................................................

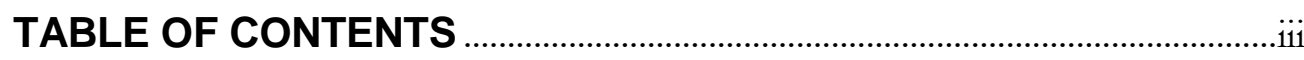

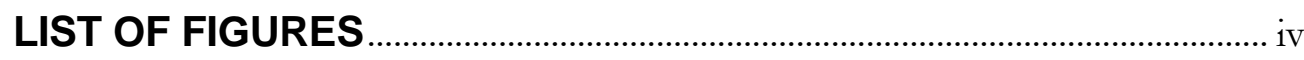

LIST OF TABLES

CHAPTER 1: INTRODUCTION...................................................................................

CHAPTER 2: CREATING A DATABASE OF STROTHER'S ARTWORKS.............9

CHAPTER 3: STROTHER'S IMAGES OF THE CANAAN VALLEY....................... 15

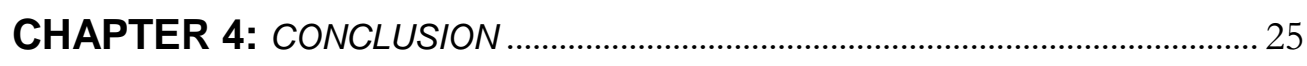

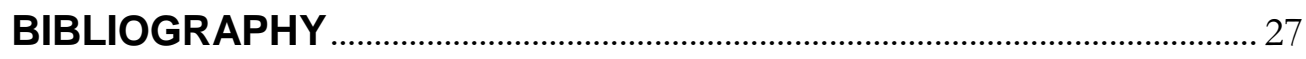

APPENDIX A: DRAWINGS OF DAVID HUNTER STROTHER IN THE WALTERS ART MUSEUM 30

APPENDIX B: ARTWORKS OF DAVID HUNTER STROTHER IN THE WEST VIRGINIA \& REGIONAL HISTORY CENTER'S DIGITAL COLLECTION. 32 


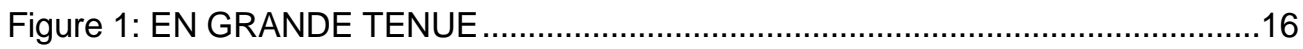

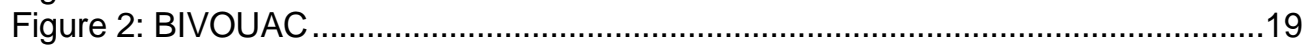

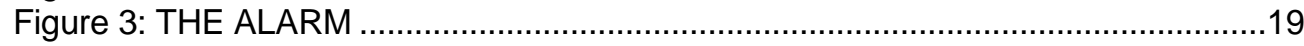

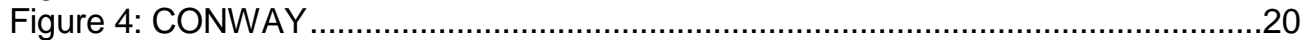

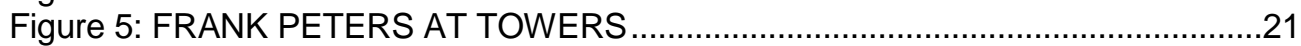

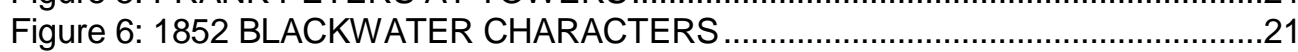

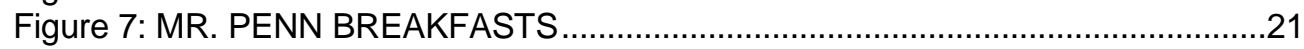

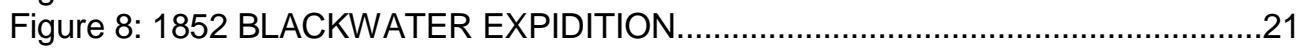

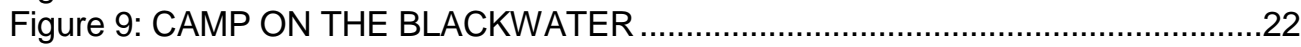

Figure 10: ENCAMPMENT ON SKILLET FORK …............................................22

Figure 11: ENCAMPMENT NO. 3 ON THE CANAAN FORK ….................................23 


\section{LIST OF TABLES}

Table 1: THEMES IN THE ARTWORK OF DAVID HUNTER STROTHER ...............14 


\section{CHAPTER1: INTRODUCTION}

In the mid-nineteenth century, subscribers of the popular Harper's New Monthly

Magazine received stories about literature, the arts, and travel which were frequently illustrated by woodcut engravings. One of the magazine's most popular illustrators during this time period was David Hunter Strother, a native of Martinsburg, Virginia (now West Virginia). ${ }^{1}$ Using the pseudonym "Porte Crayon," Strother published semi-fictional accounts of his travels which he often illustrated as well as authored. Strother's works range from the 1830s to the 1880s, which encompasses the beginning of his work as an artist, his rise in popularity as one of Harper Magazine's most popular authors and illustrators, to his final publications before his death. Strother's images of Appalachia can be found in his journals, sketchbooks, and published works from his travels throughout western Virginia and other areas in the region. ${ }^{2}$

His first published images of Appalachia were based on trips taken to Randolph County, Virginia (now West Virginia) in the summers of 1851 and 1852. The Blackwater Chronicle was an account of this trip written by his cousin and traveling companion Philip Pendleton Kennedy and illustrated by Strother. ${ }^{3}$ Strother went on to write and illustrate his own version of these trips, titled "The Virginian Canaan," in his first publication for Harper's New Monthly Magazine. 4

\footnotetext{
${ }^{1}$ John A. Cuthbert and Jessie Poesch, David Hunter Strother: "One of the Best Draughtsman the Country Possesses" (Morgantown, WV: West Virginia University Press, 1997), 11.

2 A full list of Strother's published works is available in Cecil Eby, "Porte Crayon": The Life of David Hunter Strother (Chapel Hill, NC: University of North Carolina Press, 1960), 227-231. Strother's journals and unpublished sketches are available at the West Virginia \& Regional History Center with a few additional unpublished sketches owned by the Walters Art Museum in Baltimore, MD.

${ }^{3}$ Philip Pendleton Kennedy, The Blackwater Chronicle: A Narrative of an Expedition into the Land of Canaan in Randolph County, Virginia (New York: J.S. Redfield, 1853).

${ }^{4}$ David Hunter Strother, "The Virginian Canaan," Harper's New Monthly Magazine VIII (December 1853): 18-36.
} 
Following the success of this article, Strother published a series of articles about his journeys through the Valley of Virginia. ${ }^{5}$ Continuing his literary career, Strother traveled through the south in 1857 to 1858 and wrote a series of articles that included locations such as southwestern Virginia, Chattanooga, Tennessee, and New Orleans. ${ }^{6}$ In 1858, Strother traveled through western Virginia to Ohio with a group of artists and published the article "Artists Excursion over the Baltimore and Ohio Railroad." After the Civil War, Strother returned to his work as an author and illustrator for two series featuring Virginia and the new state of West Virginia. From 1868 to 1870, he wrote a children's series called "The Young Virginians" set in the Shenandoah Valley. ${ }^{8}$ From 1872 to 1875 , he wrote a series called "The Mountains" based on his previous travels and new trips to West Virginia that featured the natural scenery and local people in the new state. ${ }^{9}$

In addition to these published works, Strother completed sketches throughout his travels. These sketches contribute to our understanding of how Strother perceived the regions he visited. The West Virginia and Regional History Center owns approximately 730 sketches and paintings created by Strother between 1833 and 1887. ${ }^{10}$ Additionally, the Walters Art Museum provides

\footnotetext{
${ }^{5}$ David Hunter Strother, "Virginia Illustrated," Harper's New Monthly Magazine (1854 to 1856). For a detailed list of the dates, volume numbers, and pages in this series see Eby's "Porte Crayon": The Life of David Hunter Strother, 227. This popular series was later re-published as David Hunter Strother, Virginia Illustrated: Containing a Visit to the Virginian Canaan and the Adventures of Porte Crayon and His Cousins (New York: Harpers and Brothers, Publishers, 1857).

${ }^{6}$ David Hunter Strother, "A Winter in the South," Harper's New Monthly Magazine (1857 to 1858). For a detailed list of the dates, volume numbers, and pages in this series see Eby's "Porte Crayon": The Life of David Hunter Strother, 228.

${ }^{7}$ David Hunter Strother, "Artist's Excursion on the Baltimore and Ohio Railroad," Harper's New Monthly Magazine XIX (June 1859): 1-19.

${ }^{8}$ David Hunter Strother, "Young Virginians," Riverside Magazine (1868 to 1870). For a detailed list of the dates, volume numbers, and pages in this series see Eby's "Porte Crayon": The Life of David Hunter Strother, 229.

${ }^{9}$ David Hunter Strother, "The Mountains," Harper's New Monthly Magazine (1872 to 1875). For a detailed list of the dates, volume numbers, and pages in this series see Eby's "Porte Crayon": The Life of David Hunter Strother, 228.

${ }^{10}$ David Hunter Strother, "Drawings of David Hunter Strother," West Virginia and Regional History Collection, West Virginia University Library, Morgantown, WV. http://images.lib.wvu.edu/s/strother/. The majority
} 
online access to the eight Strother sketches the museum owns. These collections contain many drawings that Strother made while traveling in Appalachia, New England, the American South, and Europe. Some of these sketches were used as the basis for his published illustrations. In addition to drawings he made during his travels, the collection includes numerous drawings Strother completed periodically between the 1830s and 1880s while living in Berkeley County, West Virginia.

While a number of Strother's sketches and published illustrations have been studied by art historians, the majority of his works have received minimal scholarly analysis, particularly in the context of the study of Appalachian culture. A careful study of the images created by this author and illustrator provides an avenue for understanding nineteenth-century Appalachian culture, both in what these images reveal about the people and lifestyles he encountered and in how he portrayed this region to people outside of the area.

Strother's artworks were largely ignored by scholars until the 1950s when Cecil Eby completed a doctoral dissertation on the life and work of this author. ${ }^{11}$ A condensed version of Eby's dissertation was published in 1960 as "Porte Crayon": The Life of David Hunter Strother. ${ }^{12}$ While Eby's biography still stands as the most thorough account of Strother's life and literary works, this book focuses mainly on Strother's accomplishments as an author. His visual works are largely ignored. ${ }^{13}$ In addition to this biography, Eby also wrote an article summarizing

\footnotetext{
of these images are drawings from the Strother's sketchbooks that were donated to the university by his descendants.

${ }^{11}$ Cecil Eby, "A Critical Biography of David Hunter Strother ("Porte Crayon")" (PhD diss., University of Pennsylvania, 1958).

${ }^{12}$ Cecil Eby, "Porte Crayon": The Life of David Hunter Strother (Chapel Hill, NC: University of North Carolina Press, 1960).

${ }^{13}$ This limitation was noted by art historian Jessie Poesch in "Eight Drawings by David Hunter Strother in the Walters Collection," The Journal of the Walters Art Gallery 52/53 (1994/1995): 76.
} 
Strother's experiences traveling and studying art in Europe. ${ }^{14}$ While this article focuses on Strother's training as an artist, none of the paintings or sketches he created during this trip were included in the study.

In addition to his biographical account of Strother's life, Eby sought to call attention to Strother's work by reproducing some of the author's popular publications. In 1959, Eby published The Old South Illustrated which contains reproductions of Strother's "Virginia Illustrated," "The Great Dismal Swamp," "North Carolina Illustrated," and "A Winter in the South," all of which were originally published in Harper's magazine. ${ }^{15}$ Eby also published an article that reproduces a journal Strother kept while traveling in Washington, D.C., Williamsburg, and Yorktown in $1849 .{ }^{16}$ Eby published a second book, A Virginia Yankee in the Civil War: The Diaries of David Hunter Strother, which contains Strother's personal writings while he was in the military. ${ }^{17}$

Following in the path of Eby, Jim Comstock published the Porte Crayon Sampler: David Hunter Strother in $1974 .{ }^{18}$ Comstock's book provides reproductions of "The Virginian Canaan," "Virginia Illustrated," “The Mountains," and Strother's diaries from the Civil War. A more recent reproduction of Strother's writing can be found in John Stealey's Porte Crayon's Mexico: David Hunter Strother's Diaries in the Early Porfirian Era, 1879-1885, published in 2006, which provides reproductions of the artist's journals during his time as the U.S. Consul General

\footnotetext{
${ }^{14}$ Cecil Eby, "A West Virginian in Europe: The Apprenticeship of 'Porte Crayon', 1840-1843," West Virginia History 19 (July 1958): 266-279.

${ }^{15}$ David Hunter Strother, The Old South Illustrated, ed. Cecil Eby (Chapel Hill, NC: University of North Carolina Press, 1959).

${ }^{16}$ Cecil Eby, "“Porte Crayon" in the Tidewater," The Virginia Magazine of History and Biography 67 (October 1959): 438-49. This journal was made available to Eby by a descendent of Strother who later donated the journal to the West Virginia and Regional History Center.

${ }^{17}$ David Hunter Strother, A Virginia Yankee in the Civil War: The Diaries of David Hunter Strother, ed. Cecil Eby (Chapel Hill, NC: University of North Carolina Press, 1961).

${ }^{18}$ David Hunter Strother, Porte Crayon Sampler: David Hunter Strother, ed. Jim Comstock (Richwood, WV: Jim Comstock, 1974).
} 
to Mexico. ${ }^{19}$ While these works are useful methods of making Strother's writings and illustrations available to modern audiences and calling attention to the work of this author and artist, these publications contain minimal analysis of Strother's illustrations, particularly those of Appalachia.

Strother's artistic works received a resurgence of interest in the 1990s. At the North American Print Conference in Atlanta, Georgia, several presenters focused on Strother's illustrations. The proceedings of this symposium were published in Graphic Arts and the South. Proceedings of the 1990 North American Print Conference. ${ }^{20}$ In this work, art historian Jessie Poesch contributed a chapter on "David Hunter Strother: Mountain People, Mountain Images" that discussed Strother's depictions of western Virginia. ${ }^{21}$ This chapter provides an introduction to Strother's portrayals of rural and mountain people and lifestyles. Poesch notes that Strother's works are largely unstudied because of a decline in interest in travel writing in the early twentieth-century and because his works are spread throughout various magazine publications. ${ }^{22}$ By studying his sketches and published images, Poesch provides an important summary and analysis of Strother's portrayal of rural people that shows his sensitivity to individual personalities and his interest in common people and situations.

The second historian at this conference to discuss Strother was Dana White in "Two Perspectives on the Cotton Kingdom: "Yeoman" and "Porte Crayon." 23 In this chapter, White

\footnotetext{
${ }^{19}$ David Hunter Strother, Porte Crayon's Mexico: David Hunter Strother's Diaries in the Early Porfirian Era, 1879-1885, ed. John E. Stealey, III (Kent, Ohio: Kent State University Press, 2006).

20 Judy L. Larson, ed., Graphic Arts and the South: Proceedings of the 1990 North American Print Conference (Fayetteville, AR: University of Arkansas Press, 1993).

${ }^{21}$ Jessie Poesch, "David Hunter Strother: Mountain People, Mountain Images," in Graphic Arts and the South: Proceedings of the 1990 North American Print Conference, ed. Judy L. Larson (Fayetteville, AR: University of Arkansas Press, 1993) 63-99.

22 Poesch, “Mountain People, Mountain Images," 91.

${ }^{23}$ Dana F. White, "Two Perspectives on the Cotton Kingdom: "Yeoman" and "Porte Crayon"," in Graphic Arts and the South: Proceedings of the 1990 North American Print Conference, ed. Judy L. Larson (Fayetteville, AR: University of Arkansas Press, 1993) 101-127.
} 
compares Strother's works with those of Frederick Law Olmstead, who traveled in the south during the same time period as Strother and wrote under the pseudonym "Yeoman." White concludes that while Strother's narratives and illustrations were entertaining to contemporary readers, his works had little appeal to later audiences because they lacked the depth of Olmsted's accounts. While White included several of Olmsted's publications, Strother's only publication discussed in this chapter was from his travels in the Dismal Swamp in coastal Virginia and North Carolina, leaving the majority of his sketches and published images unexplored.

A third historian at this conference, Gregg Kimball, discussed Strother in the chapter "The South as It Was: Social Order, Slavery, and Illustrators in Virginia, 1830-1877."24 Kimball provides an analysis of how illustrators portrayed southern African Americans in images of plantation life, slave auctions, urban slavery, and freedom. He includes works by Strother from his travels in Richmond, Virginia, the Tidewater region of Virginia, and Harper's Ferry, Virginia (now West Virginia). He concludes that illustrators such as Strother created images that reflected their own and their readers' fears of urbanization and African American freedom. ${ }^{25}$

Jessie Poesch, continuing her interest in Strother's illustrations, analyzed eight of Strother's drawings owned by the Walters Art Museum in Baltimore, Maryland. ${ }^{26}$ These drawings are sketches Strother completed in the late 1850s and sold to the museum's founder William T. Walters. These works are similar to Strother's drawings in the West Virginia and Regional History Center's collection. Poesch provides a thorough analysis of these drawings, often by comparing these sketches to their corresponding published illustrations. While this

\footnotetext{
${ }^{24}$ Gregg Kimball, "The South as It Was: Social Order, Slavery, and Illustrators in Virginia, 1830-1877"," in Graphic Arts and the South: Proceedings of the 1990 North American Print Conference, ed. Judy L. Larson (Fayetteville, AR: University of Arkansas Press, 1993) 129-157.

${ }^{25}$ Gregg Kimball, "The South as It Was," 146.

${ }^{26}$ Jessie Poesch, "Eight Drawings by David Hunter Strother in the Walters Collection," The Journal of the Walters Art Gallery 52/53 (1994/1995): 65-77.
} 
article is an excellent analysis of the works studied, this represents only eight of the hundreds of images Strother created.

In 1997, Poesch collaborated with John Cuthbert, curator at the West Virginia and Regional History Center, to co-curate an exhibit of Strother's drawings. Poesch and Cuthbert wrote a catalogue to accompany the exhibit. ${ }^{27}$ In this catalogue, Cuthbert provides a biography of Strother while Poesch analyzes the forty-two sketches in the exhibit. ${ }^{28}$ Poesch again provides a thorough analysis of the drawings under study. Cuthbert also makes significant points about Strother's artworks throughout the biography. However, seeking to portray the range of Strother's works, this analysis includes only a small fraction of the images he created, ranging across the timespan of his life and the various regions he visited. Additionally, in focusing on the sketches in the collection, the authors at times do not fully compare these works with their corresponding published illustrations.

In a recent article published in 2013, Michael S. Martin studied the role of maps in Kennedy's The Blackwater Chronicle and Strother's "The Virginian Canaan."29 Martin compared these two works to show that both authors abandoned conventional notions of cartographic knowledge in favor of a first-person, experiential understanding of the terrain. While the primary focus of this article was a literary analysis of the texts, Martin briefly discusses Strother's illustrations. He argues that these images were used to reinforce the personal experiences of the travelers and the authors' rejection of conventional mapping methods.

\footnotetext{
${ }^{27}$ John A. Cuthbert and Jessie Poesch, David Hunter Strother: "One of the Best Draughtsman the Country Possesses" (Morgantown, WV: West Virginia University Press, 1997).

${ }^{28}$ Cuthbert also wrote a summary of Strother's works and a brief biography in Early Art and Artists in West Virginia: An Introduction and Bibliographical Directory (Morgantown, WV: West Virginia University Press, 2000): 19-25 (portraits), 73-18 (illustrations and genre paintings), and 241-241 (biography).

${ }^{29}$ Michael S. Martin, "'The Wilderness was Growing Wilder": The Limits of Cartographic Knowledge in Philip Pendleton Kennedy's The Blackwater Chronicle and David Hunter Strother's The Virginia Canaan," Journal of Appalachian Studies 19 (2013): 46-58.
} 
While Strother's works have received attention from scholars since the 1950's, this attention has been sporadic and of varied methods and purpose. The most frequent work on Strother has been in the form a biographies and reproductions of his writings. While these biographies and reproductions are useful, they often provide little analysis of Strother's works and tend to focus on his literary rather than artistic accomplishments. Some scholarly analysis, mainly done in the 1990s, has been completed on his artistic works. However, the works under study tend to focus on his published illustrations and rarely focus on his images of Appalachia. Significant analysis of Strother's drawings has been conducted by art historian Jessie Poesch and John Cuthbert. However, this analysis still represents less than ten percent of Strother's known drawings. ${ }^{30}$

Strother's numerous images, both his published works and his private sketchbooks, present an opportunity to explore nineteenth-century American culture through the eyes of this artist. Of the over seven hundred available images in the West Virginia Regional History Center's collection, only a small portion of these works have received careful analysis in scholarly publications. This collection was digitized and placed online in 2001, making the collection highly accessible. ${ }^{31}$ However, the organization of this collection alphabetically by title makes chronological, geographic, or thematic studies of Strother's works difficult. Methods for organizing, presenting, and studying these collections were developed as part of this study to improve the accessibility of these resources.

\footnotetext{
${ }^{30}$ Poesch and Cuthbert's exhibit catalogue David Hunter Strother: "One of the Best Draughtsman the Country Possesses" includes 61 drawings from this collection. Poesch also included 10 of these works in her book chapter "Mountain People, Mountain Images," and one drawing in her article "Eight Drawings by David Hunter Strother in the Walters Collection." Cuthbert includes five of these drawing in Early Art and Artists in West Virginia. There is some repetition of the images used in these publications

${ }^{31}$ David Hunter Strother, "Drawings of David Hunter Strother," West Virginia and Regional History Collection, West Virginia University Library, Morgantown, WV. http://images.lib.wvu.edu/s/strother/.
} 


\section{CHAPTER 2: CREATING A DATABASE OF STROTHER'S ARTWORKS}

A database was created to provide a method for studying Strother's artworks (see Appendix A and Appendix B). ${ }^{32}$ The creation of a database of Strother's artworks enables increased study and understanding of these works. In addition to the West Virginia and Regional History Center's collection, the eight pieces in the Walters Art Museum are included in the database to provide a thorough summary of Strother's sketches. The creation of a database is an effective method of determining patterns within the artist's work which are often not readily apparent such as travel patterns and themes within the artist's works.

The first step in making this collection more accessible was to identify key elements in each work such as the date the sketch was made and the location of the subject. In order to make the database, each digital image was reviewed for information. Strother often wrote notes on his sketches to denote the date and location of the sketch. He occasionally included names of individuals and other descriptive details. The West Virginia and Regional History Center's collection uses Strother's notes as the title for the image. However, the transcription of Strother's notes often contain errors such as misspellings of locations. ${ }^{33}$ While Strother's notes sometimes contain dates and locations, often one or both pieces of information are missing. Therefore, these items are missing in the titles and unavailable in searches of the collection. Over 200 images are listed simply as "Untitled" because there are no notes on the sketch. Identifying this information makes searching the collection easier and reveals connections between works. The final database

\footnotetext{
32 The database was placed on a website (www.davidhunterstrother.omeka.net) to make the information easily available. On this site, sketches can be searched alphabetically, by date, by location on a map, or by thematic tags.

${ }^{33}$ The titles used in the database are identical to the titles in the digital collections to ensure that the correct images are referenced, although the punctuation is unusual at times.
} 
shows the date sketches were created and the location the sketch depicts (when they could be determined), as well as important themes within the works.

To determine the date for each sketch, information was taken from the writing on the sketch, when present, as the most reliable source. If no date was present or was occasionally illegible, then the date was taken from the West Virginia and Regional History Center's Description Page for the image in the digital collection, although these dates were sometimes found to be incorrect. The source of the information for the date is provided in the database so that this information can be evaluated by users. By studying each image, it was possible to clarify the dates for some images when the writing on the sketch is unclear. For example, the date for the sketch titled "Ken Foster. Black Mountains. East Tenn," (Number 297), was determined by comparing this image to other sketches Strother completed of his travels in Tennessee in order to find the correct date of the sketch. Dates were also found by using information in Cecil Eby’s biography of Strother's life. ${ }^{34}$

Observation of each image enabled the identification of locations for a majority of the sketches. Strother often noted the town or county, without stating a specific state. Locations could often be confirmed by comparing sketches, each with partial information, and using primary sources and secondary literature. For example, cross referencing images was used to determined missing information for the locations of Ore Knob in Guilford, NC (Numbers 181 and 266) and Aaron Armantrout's house in Randolph County (Numbers 15 and 285). Reference sources were found to confirm the locations such as the Glen House in White Mountains, New Hampshire (http://whitemountainhistory.org/Glen_House.html). Confirming the location noted on sketches made it is possible to group these images with other sketches from his New

\footnotetext{
${ }^{34}$ Cecil Eby, "Porte Crayon": The Life of David Hunter Strother (Chapel Hill, NC: University of North Carolina Press, 1960).
} 
Hampshire trip. It was also possible to determine the location of some images using Strother's publication such as his sketch of a local Berkeley Springs man he wrote about in "The Bear and the Basket Maker"35 (Number 245).

Because the handwriting on the sketches is difficult to read, at times the West Virginia and Regional History Center transcribes the date on the images partially or inconsistently. Creating the database allowed for correcting inconsistencies in the transcriptions of locations. For example, the town of Martinsburg was spelled variously as Marinsburg, Martinsburg, and Maarinsburg (Numbers 325, 326, and 430). Using a consistent name increases the likelihood of associating all related images. Clarifying the locations also allowed for distinguishing between Charleston and the more frequently sketched Charles Town, especially in the Strother's sketches of the trial of abolitionist John Brown. By making these corrections, we can ensure that all of the related sketches are grouped together for study.

A central component of the database is the identification of themes within Strother's works (see Table 1). Certain themes predominate throughout his works and have the potential to provide a useful method of analysis. As each sketch was reviewed, applicable themes were noted in the database. Some themes are simple categories, such as gender, that can be used to compare similar images. The themes of "Men" and "Women" were further broken down into activities such as "Men/Women at Leisure," "Men/Women at Work," and "Men/Women in Wilderness." Leisure activities included items such as sleeping, reading, or smoking. Work activities included items such as mining, driving a carriage, or farming. The theme of being in the wilderness was generally defined as a person in the forest or mountains, without any visible man-made

\footnotetext{
${ }^{35}$ Strother, "The Bear and the Basket Maker," Harpers New Monthly Magazine, June 1856.
} 
landscaping, roads, or buildings. Strother made numerous sketches of African Americans and children, so these were included as themes as well.

Strother's vocation as a travel writer is reflected in the themes. As a travel writer, movement was central to Strother's works. The theme of "Transportation" includes various methods of transportation such as boats, railroads, and carriages (which also broadly includes small carts and large covered wagons). Strother also documented the various landscapes he encountered on his travels such as lakes, mountains, caves, rivers, and waterfalls. Strother also documented the domestic and wild animals he encountered.

In addition to these elements, Strother sketched the architecture, decorative arts, religious shrines, and cemeteries he encountered. Architecture included institutional buildings such as schools, churches, and government buildings and residential buildings such as houses and farms. Decorative arts items ranged from high style goods to simple household furnishings or possessions that reflect local or contemporary culture. He sketched and painted the religious shrines he visited while studying art in Italy and the cemeteries he saw in the southern states and Mexico. Strother also occasionally sketched and painted copies of European and Japanese artworks.

Strother's works also reflect the events and developments of the time period. He drew the emerging industries he saw on his travels such as his sketches of miners in Guilford, North Carolina (Number 181), the salt works in southwestern Virginia (Numbers 253, 434, and 435), an ironworks in Tennessee (Number 265), and sugar production in Louisiana (Numbers 471 to 474). Strother also drew scenes from his time in the military and his observation of the Harpers Ferry Raid Trials. By labeling all of Strother's sketches of the Harpers Ferry Raid Trials, it was 
possible to identify numerous images related to the trials that are scattered throughout the collection.

To show the utility of the database, Strother's sketches from his 1852 trip to the Canaan Valley, along with his published article about the trip, were analyzed. By identifying dates, locations, and themes within Strother's sketches, it is possible to see travel patterns within his works such as his trip to the Canaan Valley. Studying these sketches as a group reveals perspectives that are missing from his published article, showing how he actively characterized the region as a beautiful but rugged wilderness whose inhabitants were skilled but simple outdoorsmen. 
TABLE 1: THEMES IN THE ARTWORK OF DAVID HUNTER STROTHER

\begin{tabular}{|l|l|}
\hline African Americans & Men: \\
Animals: & Men at Leisure \\
$\quad$ Domestic & Men at Work \\
Wild & Men in Wilderness \\
Architecture: & Military \\
$\quad$ Institutional Buildings & Religious Shrines \\
$\quad$ Residential Buildings & Transportation: \\
Towns & Boats \\
$\quad$ Bridges & Carriages \\
Cemeteries & Railroads \\
Children & Women: \\
Copies of European Artwork & Women at Leisure \\
Copies of Japanese Artwork & Women at Work \\
Decorative Arts & Women in Wilderness \\
Harper's Ferry Raid Trials & \\
Industry & \\
Landscape: & \\
Caves & \\
$\quad$ Fences & \\
$\quad$ Lakes & \\
$\quad$ Mountains & \\
\hline Rivers & \\
\hline
\end{tabular}


"In Randolph County, Virginia, is a tract of country containing from seven to nine hundred square miles, entirely uninhabited, and so savage and inaccessible that it has rarely been penetrated even by the most adventurous." - David Hunter Strother, $1853 .^{36}$

Strother's magazine article, titled "The Virginian Canaan," about his trip to the area in the summers of 1851 and 1852, includes twenty engravings. ${ }^{37}$ Using the database of Strother's sketches, twenty-five images of the Canaan Valley were identified. An analysis of Strother's images of the Canaan Valley, both in sketches and the engravings that accompanied his published story, reveal his attitudes toward the region as a dangerous but beautiful land inhabited by noble but simple outdoorsmen.

In his article, Strother presents a group of friends traveling from Martinsburg, Virginia (now West Virginia) to the Canaan Valley for a hunting and fishing expedition. ${ }^{38}$ The valley includes the headwaters of the Blackwater River and the Blackwater Falls. The Canaan Valley remains a large outdoor recreation area within the state. ${ }^{39}$ However, nineteenth-century travelers would have seen a very different forest than the one that exists today. The old growth forests that covered the state were almost entirely logged by the timber industry in the $1890 \mathrm{~s}-1920 \mathrm{~s}^{40}$

\footnotetext{
${ }^{36}$ Strother, "The Virginian Canaan," 18.

37 Strother, "The Virginian Canaan," Harper's New Monthly Magazine VIII (December 1853): 18-36.

${ }^{38}$ Strother's characters are based on his real-life friends and traveling companions, but they are each given pseudonyms, even Strother, who refers to himself as Mr. Porte Crayon. Strother, "The Virginian Canaan," 18.

${ }^{39}$ Various portions of the Monongahela National Forest, the Canaan Valley State Park, and Blackwater Falls State Park are within Canaan Valley.

${ }^{40}$ Ronald Lewis, Transforming the Appalachian Countryside: Railroads, Deforestation, and Social Change in West Virginia 1880-1920 (Chapel Hill and London: University of North Carolina Press, 1998).
} 


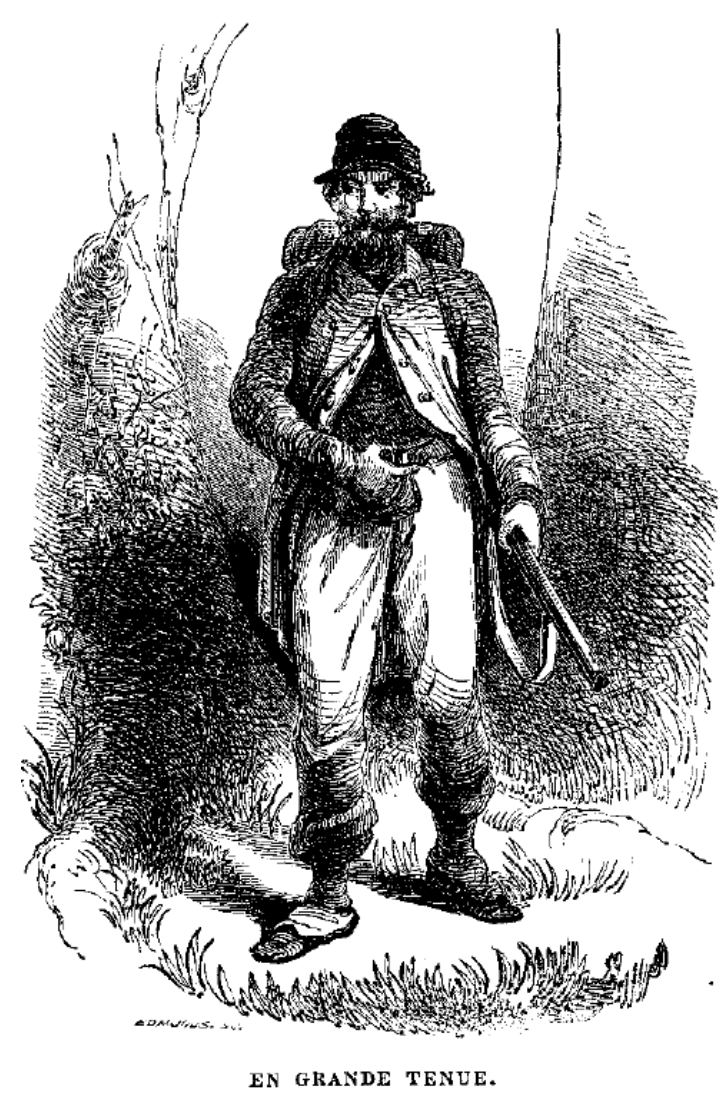

Figure 1. "En Grande Tenue," in “The Virginian Canaan," Harper's New Monthly Magazine VIII (December 1853): 18.

Strother's images show a consistent pattern of representing the Canaan Valley as a dangerous, uninhabited wilderness. The opening description of the group's plans to travel to the Canaan Valley establishes the region as an area of danger. The men are attracted by what Strother call the region's "savage grandeur" and the area is described as "entirely uninhabited.",41 Strother emphasizes the danger of the region by using military terminology throughout the article. He states, "A council of war decided that the army was not in condition to move on that

\footnotetext{
${ }^{41}$ Strother, "The Virginian Canaan," 18.
} 
day, and that they should remain under cover, and repose while such as felt disposed should go out as scouts to explore thoroughly the surrounding country." 42

He also focuses on the extent of preparation needed for the trip. ${ }^{43}$ The travelers dress themselves like French soldiers shown in the illustration "En Grande Tenue" (Figure 1). This figure is shown standing in a wooded area dressed in informal work clothes. That Strother felt it worthwhile to depict his clothing and equipment for the trip, particularly his gun and backpack, suggest that he was diverging from the everyday norms of his readers.

Using the database, it is possible to analyze Strother's works by studying the artist's process from sketch to completed illustration. This method involves tracking the development of Strother's illustrations from preliminary sketches in his sketchbooks to the completed illustrations in his published works. ${ }^{44}$ This method of analysis shows which initial sketches were or were not selected for further development and publication, showing what was considered suitable for mass marketing either by Strother or his publishers. Looking at how these images changed from sketch to publication shows the influence and demands of the publisher and the expectations of public audiences.

Comparing the images in the published article with Strother's sketches of his trips shows that while some sketches are very similar to the published versions, there are often notable differences between his sketches and published versions, as well as many sketches that were not used in the final publication. The sketch "Bivouac (Virginia Canaan) June 1852" (Number 107)

${ }^{42}$ Strother, "The Virginian Canaan," 28.

${ }^{43}$ Strother, "The Virginian Canaan," 19-20.

${ }^{44}$ This method was used by David Sellin in American Art in the Making: Preparatory Studies for Masterpieces of American Painting, 1800-1900 (Washington D.C.: Smithsonian Institution Press, 1976) to show the development of artistic works from sketch to completed painting. Cuthbert and Poesch also use this method at times to show how the publication process affected the development of Strother's works (Cuthbert and Poesch, David Hunter Strother: "One of the Best Draughtsman the Country Possesses," 27 and Poesch, "Eight Drawings by David Hunter Strother in the Walters Collection," 67-68 and 72). 
was used as a basis for his engraving "The Alarm" (Figures 2 ad 3). ${ }^{45}$ These images show the group of men around a campfire in a forest at night, surrounded by large old growth spruce trees. The men are reacting to an unknown sound in the night. While the two images are similar, there are a few differences. In the published image, the figures are grouped more closely together and less of the surrounding forest is shown. The edge fades into the darkness of the night. These changes heighten the fear the image is striving to capture of these men encountering a potentially dangerous wilderness. Even the changing of the name from "Bivouac" to "The Alarm" suggests an increase in danger. Only the two local guides remain calm, one shown laying near the front of the image and the other smoking a pipe. They are moved closer together with the other figures but remain unchanged otherwise.

The two local guides who accompany the men on their trip to the Canaan Valley, Conway and Thornhill, are repeatedly praised for their aptitude at hunting, fishing, building shelters, and other outdoor skills. Strother states, "Conway's woodcraft...triumphed over all difficulties" 46 and "Conway's talents were again called in requisition to extend and improve the comfort of their quarters." ${ }^{, 47}$ Strother's portrayal of the guides, especially their resourcefulness and skill, and even their intelligence, is generally positive. However, the guides are consistently shown in the role of servants being ordered to attend to tasks.

\footnotetext{
${ }^{45}$ Strother, "The Virginian Canaan," 24.

${ }^{46}$ Strother, "The Virginian Canaan," 27.

${ }^{47}$ Strother, "The Virginian Canaan," 28.
} 

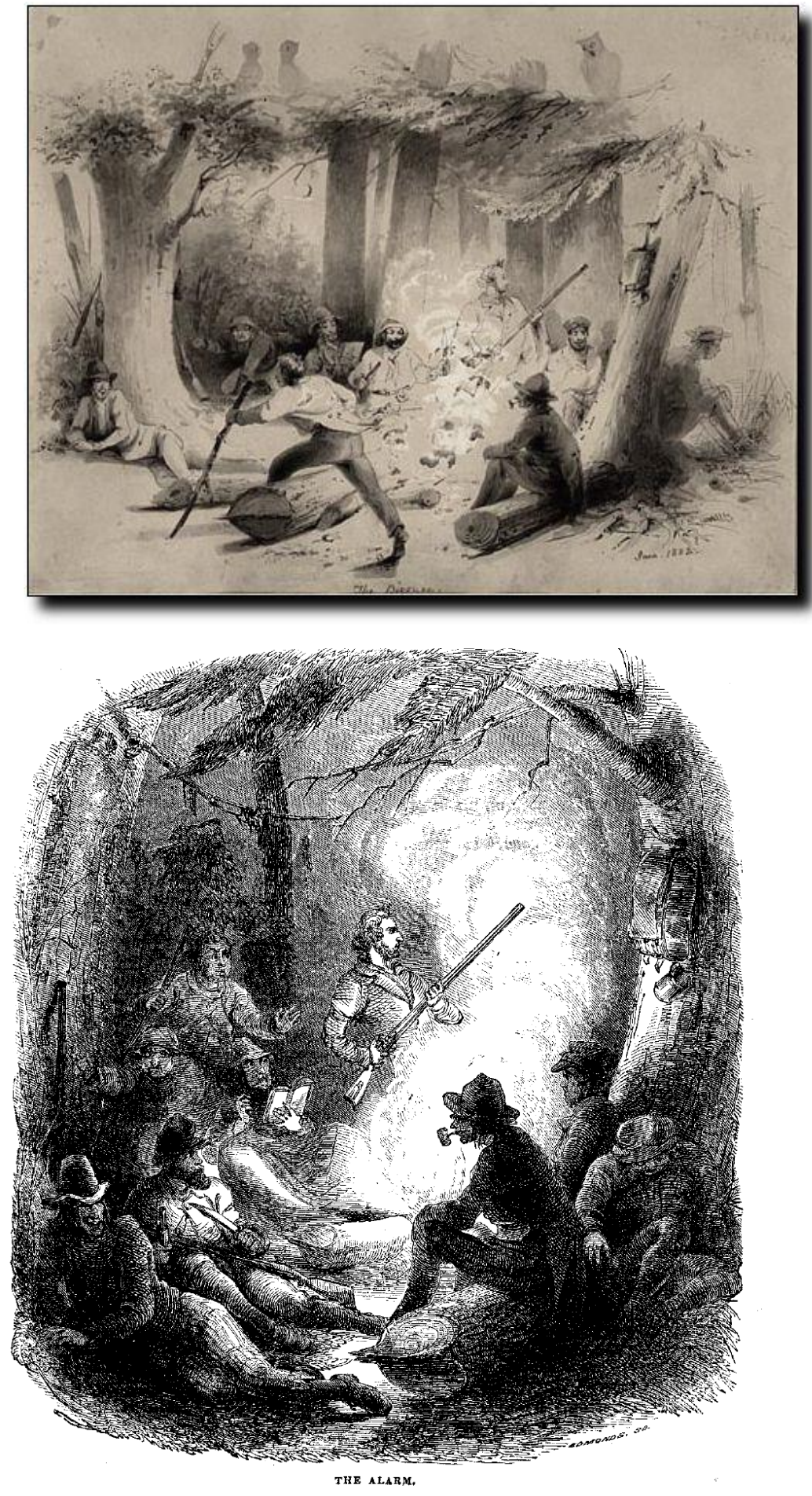

Figure 2 (Top). "Bivouac (Virginia Canaan) June 1852” (WVRHC Number 107).

Figure 3 (Bottom). "The Alarm” in “The Virginian Canaan," Harper's New Monthly Magazine VIII (December 1853): 24. 
Strother includes the portrait of one of the guides, Conway (Figure 4). This image shows the guide holding a knife, straddling a log, and surrounded by laurel bushes. His portrait is less of a caricature than the images of the travelers, showing more defined facial features and individual characteristics. This portrait shows the guide's comfort in the forest in his relaxed manner. This image is intended to contrast with the experiences of the urban readers. This published image is different than the sketches of local people Strother drew during his trip. Several sets of sketches in the West Virginia and Regional History Collection include people Strother labeled as "Blackwater Characters" (se Figures 5 and 6). These sketches contain numerous men with highly detailed facial features, expressions, and clothing, some with simple work clothes and others with more formal coats and jackets. Strother may have left these sketches out of the published story to make the region appear less inhabited in order to emphasize the danger of their trip to a remote region.

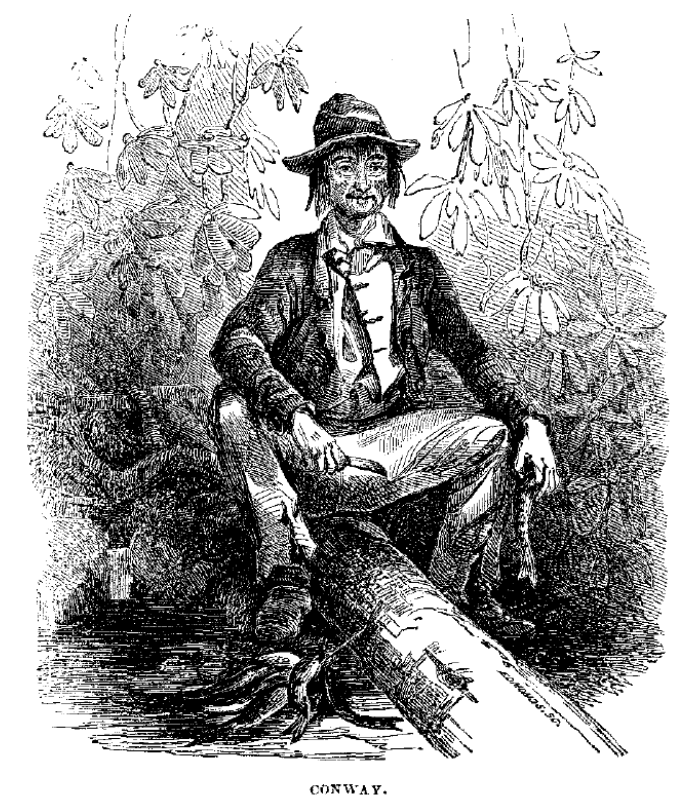

Figure 4. "Conway" in “The Virginian Canaan," Harper's New Monthly Magazine VIII (December 1853): 22. 

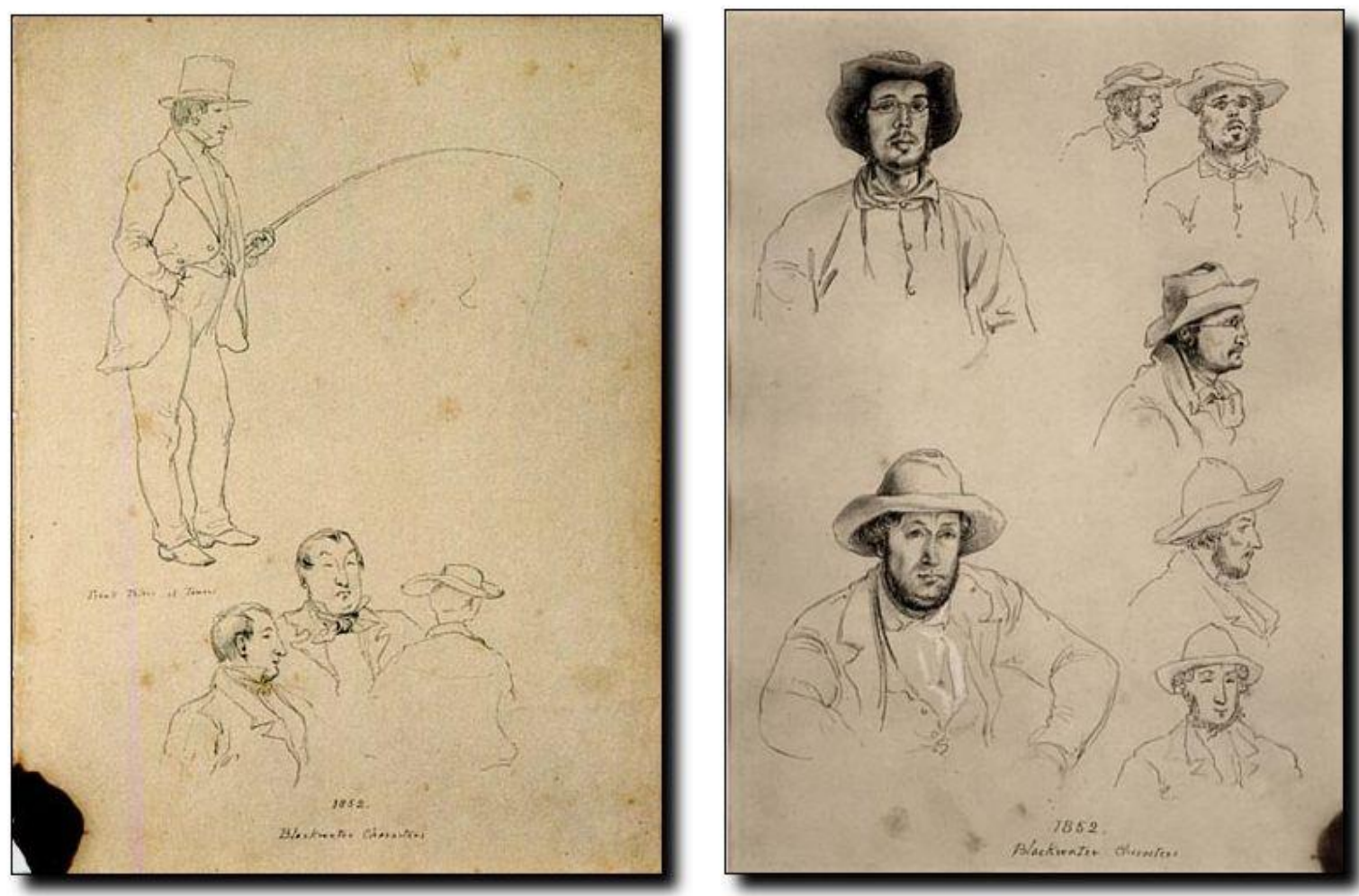

Figure 5 (Left). "Frank Peters at Towers" (WVRHC Number 218).

Figure 6 (Right). “1852 Blackwater Characters” (WVRHC Number 7).
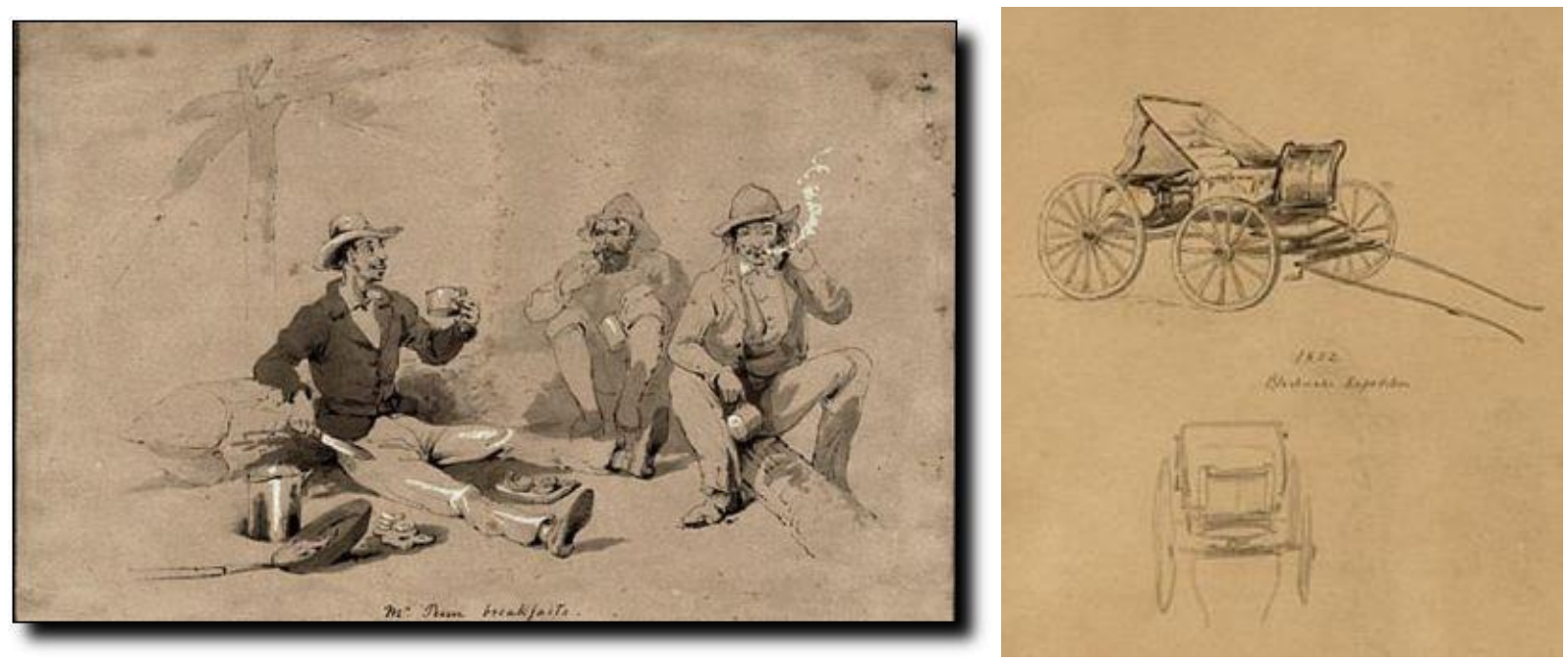

Figure 7 (Left). “Mr. Penn breakfasts” (WVRHC Number 352).

Figure 8 (Right). “1852 Blackwater Expedition” (WVRHC Number 8). 
The published article typically did not include engravings of sketches that portrayed the travelers as competent or comfortable in the forest. For example, "Mr. Penn's Breakfast” (Figure 7) shows the men enjoying a meal around a campfire. The article also did not include sketches from the trip that would appear familiar such as Strother's drawing of the carriages the men used on their trip (Figure 8). The article also omitted sketches that contradicted with the idea of the Canaan Valley as a remote, uninhabited land. For example, the published article included an image of a rustic lean-to that the men stayed in which was nearly identical to one of Strother's sketches (Figures 9 and 10). But the article did not include an engraving of his sketch of the less rustic, more skillfully crafted (and likely more comfortable) shelter (Figure 11).
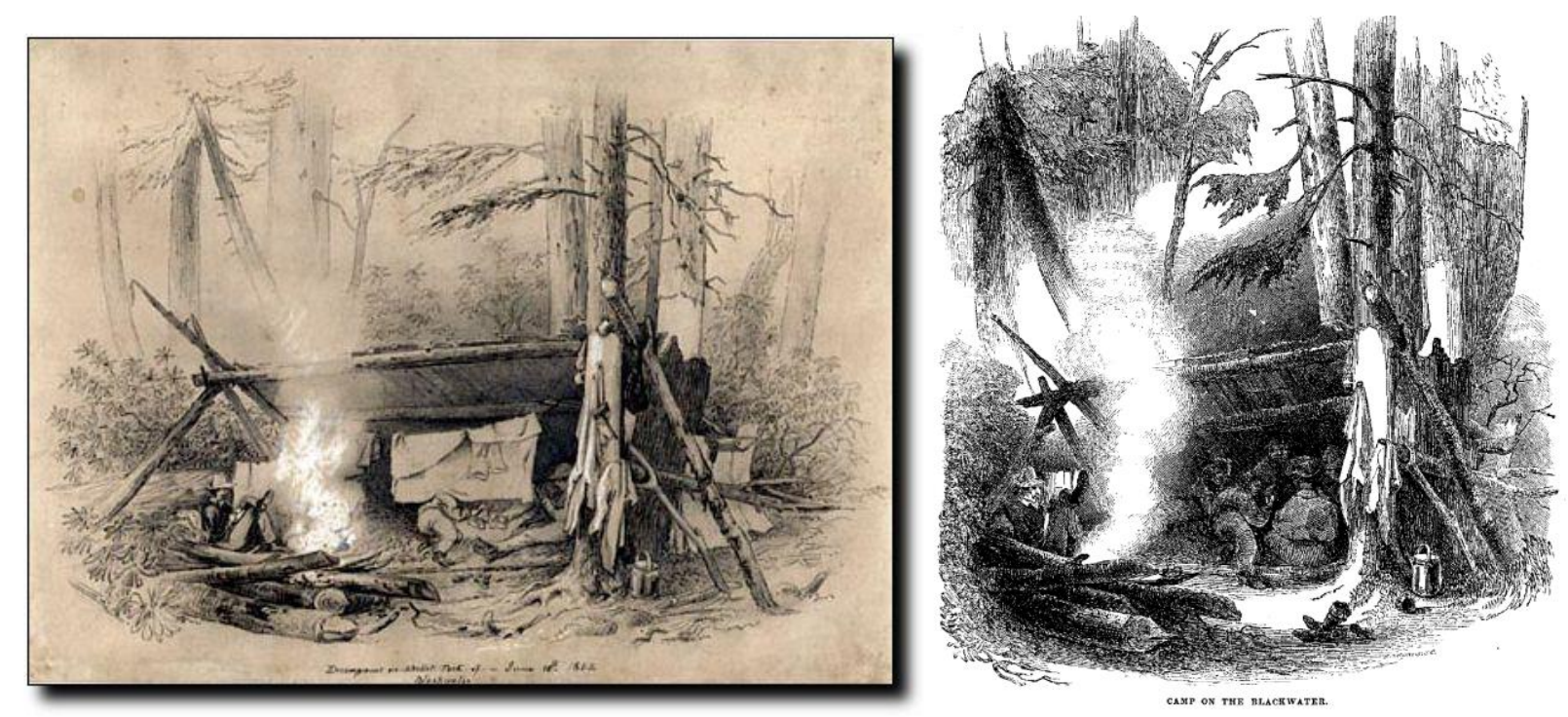

Figure 9 (Left). "Encampment on Skillet Fork (Virginia Canaan 1852)” (WVRHC Number 199). Figure 10 (Right). “Camp on the Blackwater,” in “The Virginian Canaan,” Harper's New Monthly Magazine VIII (December 1853): 34. 


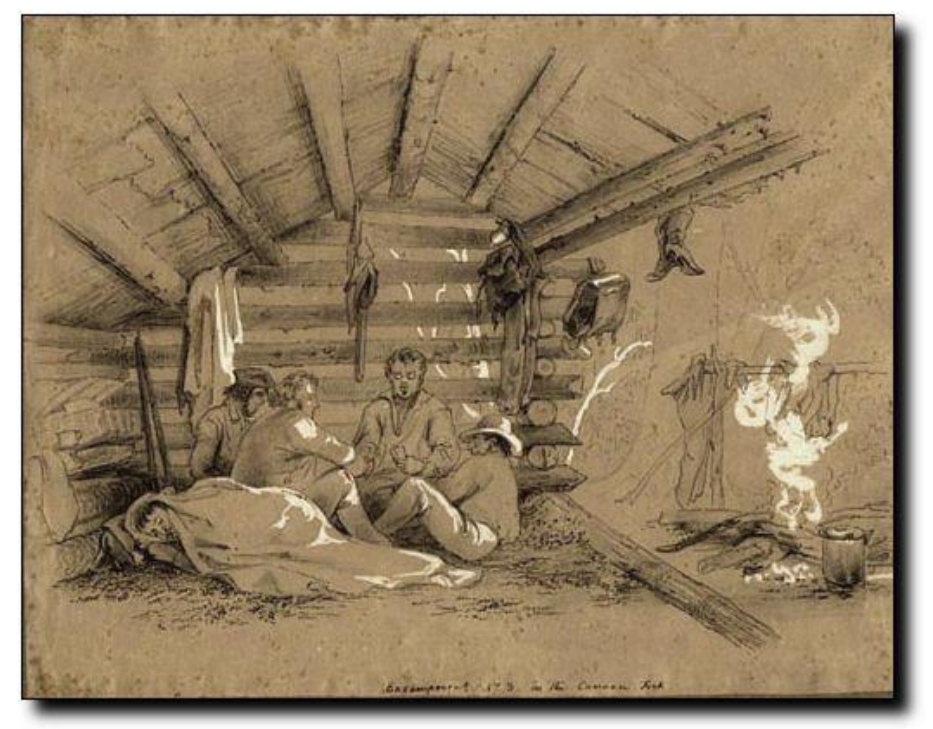

Figure 11. "Encampment No 3. on the Canaan Fork” (WVRHC Number 194).

Strother's depiction of the Canaan Valley as an uninhabited wilderness with only a few woodsmen as guides heightened the excitement of the story for his audience, emphasizing the danger of their experiences and the seclusion of the region. However, these choices are not benign. Appalachia has long been associated with popular stereotypes of the region's people as uneducated and uncivilized. ${ }^{48}$ In the past several decades, scholars of Appalachian studies have investigated how these stereotypes developed. ${ }^{49}$ Late nineteenth and early twentieth-century authors who portrayed Appalachia often used stereotypical images. Understanding how and when these stereotypes developed is important in the study of Appalachia culture.

Strother's antebellum-era images shows elements of the developing stereotypes that came to dominate perceptions of Appalachia in the twentieth century. His choices have the effect of delineating the region as a place that is in contrast to the familiar world of the reader. The

\footnotetext{
${ }^{48}$ Kenneth W. Noe, "Appalachia before Mr. Peabody: Some Recent Literature on the Southern Mountain Region," The Virginia Magazine of History and Biography 110 (2002): 5.

${ }^{49}$ Noe, "Appalachia before Mr. Peabody," 16, 21-23.
} 
Canaan Valley and its inhabitants were characterized as being distinctly different than other regions, such as eastern Virginia. While we do not know whether it was Strother or his publisher who influenced these choices, his published images were intended to appeal to the urban readers of Harper's New Monthly Magazine. Looking at Strother's works helps us to understand how stereotypes of Appalachia began developing during the mid-nineteenth century. 


\section{CHAPTER 4: CONCLUSION}

Strother's sketches and paintings depict the people, objects, and locations of his travels and everyday experiences. The current study shows the importance of advanced organizational methods for studying historical art collections. The database that was created for this project reveals connections between the artist's works based on date, location, and thematic content to provide additional methods for studying Strother's artworks. By clarifying dates and locations, connections can be made within the hundreds of digital images available online. Providing information about relevant themes within each artwork can assist in studying issues such as gender, race, and the environment in nineteenth century artworks. In addition to qualitative studies of Strother's works, the database can also be used for quantitative analysis to answer questions such as how often Strother portrayed certain topics within a region and whether his preference for certain themes changed by location or time period. Using these types of approaches, the database provides a means for studying Strother's artworks to understand the significance of these works within various contexts.

Using the database of Strother's artworks, numerous sketches were identified from Strother's Canaan Valley trip that revealed his views, or perhaps the engravers or publishers views, about the local people and the region. Future projects to continue the study of Strother's artworks would be to compare his images of Appalachia to his portrayals of other locations in America and Europe. In addition to his travels in western Virginia and the mountain regions of Tennessee, Strother also traveled to other areas in the south, New England, and Europe. He made drawings through these trips and published several articles and books based on his travels. Strother's works from these various regions can be compared to see how he portrayed various regions. This type of comparative analysis can show whether he used any distinct methods to characterize Appalachian regions. 
Another avenue of analysis would be to compare Strother's portrayals of Appalachia to how other artists of the time period portrayed the region to see how his works were similar or different from these artists. Strother created his images of Appalachia within a community of artists working in the region. ${ }^{50}$ Portrait painters, landscape painters, and other illustrators were working throughout Appalachia in the nineteenth century. Using these methods, Strother's sketches and illustrations of nineteenth-century Appalachia can be thoroughly analyzed and placed within the context of Strother's other works and the work of other artists in the region.

${ }^{50}$ Cuthbert provides a wealth of information about this community in his book Early Art and Artist's in West Virginia. 


\section{Bibliography}

Cuthbert, John A. Early Art and Artists in West Virginia: An Introduction and Bibliographical Directory. Morgantown, WV: West Virginia University Press, 2000.

Cuthbert, John A. and Jessie Poesch. David Hunter Strother: "One of the Best Draughtsman the Country Possesses.” Morgantown, WV: West Virginia University Press, 1997.

Eby, Cecil. “A Critical Biography of David Hunter Strother ("Porte Crayon").” PhD diss., University of Pennsylvania, 1958.

Eby, Cecil. "Porte Crayon”: The Life of David Hunter Strother. Chapel Hill, NC: University of North Carolina Press, 1960.

Eby, Cecil. “"Porte Crayon” in the Tidewater.” The Virginia Magazine of History and Biography 67 (October 1959): 438-49.

Eby, Cecil. “A West Virginian in Europe: The Apprenticeship of 'Porte Crayon', 1840-1843.” West Virginia History 19 (July 1958): 266-279.

[Kennedy, Philip Pendleton.] The Blackwater Chronicle. A Narrative of an Expedition into the Land of Canaan in Randolph County, Virginia. New York: J.S. Redfield, 1853.

Kimball, Gregg. "The South as It Was: Social Order, Slavery, and Illustrators in Virginia, 18301877." In Graphic Arts and the South: Proceedings of the 1990 North American Print Conference, edited by Judy L. Larson, 129-157. Fayetteville, AR: University of Arkansas Press, 1993.

Larson, Judy L., ed. Graphic Arts and the South: Proceedings of the 1990 North American Print Conference. Fayetteville, AR: University of Arkansas Press, 1993.

Lewis, Ronald. Transforming the Appalachian Countryside: Railroads, Deforestation, and 
Social Change in West Virginia, 1880-1920. Chapel Hill and London: University of North Carolina Press, 1998.

Martin, Michael S. “"The Wilderness was Growing Wilder”: The Limits of Cartographic Knowledge in Philip Pendleton Kennedy's The Blackwater Chronicle and David Hunter Strother's The Virginian Canaan.” Journal of Appalachian Studies 19 (2013): 46-58.

Noe, Kenneth W. "Appalachia before Mr. Peabody: Some Recent Literature on the Southern Mountain Region." The Virginia Magazine of History and Biography 110 (2002): 5-34.

Poesch, Jessie. "David Hunter Strother: Mountain People, Mountain Images.” In Graphic Arts and the South: Proceedings of the 1990 North American Print Conference, edited by Judy L. Larson, 63-99. Fayetteville, AR: University of Arkansas Press, 1993.

Poesch, Jessie. "Eight Drawings by David Hunter Strother in the Walters Collection.” The Journal of the Walters Art Gallery 52/53 (1994/1995): 65-77.

Sellin, David. American Art in the Making: Preparatory Studies for Masterpieces of American Painting, 1800-1900. Washington D.C.: Smithsonian Institution Press, 1976.

Strother, David Hunter. “Drawings of David Hunter Strother.” A\&M 2894. West Virginia and Regional History Center, West Virginia University Library, Morgantown, WV. http://images.lib.wvu.edu/s/strother/.

Strother, David Hunter. The Old South Illustrated. Edited by Cecil Eby. Chapel Hill, NC: University of North Carolina Press, 1959.

Strother, David Hunter. Porte Crayon's Mexico: David Hunter Strother's Diaries in the Early Porfirian Era, 1879-1885. Edited by John E. Stealey, III. Kent, Ohio: Kent State University Press, 2006.

Strother, David Hunter. Porte Crayon Sampler: David Hunter Strother. Edited by Jim Comstock. 
Richwood, WV: Jim Comstock, 1974.

Strother, David Hunter. Virginia Illustrated: Containing a Visit to the Virginian Canaan and the Adventures of Porte Crayon and His Cousins. New York: Harpers and Brothers, Publishers, 1857.

Strother, David Hunter. A Virginia Yankee in the Civil War. The Diaries of David Hunter Strother. Edited by Cecil Eby. Chapel Hill, NC: University of North Carolina Press, 1961.

White, Dana F. “Two Perspectives on the Cotton Kingdom: "Yeoman” and "Porte Crayon”." In Graphic Arts and the South: Proceedings of the 1990 North American Print Conference, edited by Judy L. Larson, 101-127. Fayetteville, AR: University of Arkansas Press, 1993. 
APPENDIX A: DRAWINGS OF DAVID HUNTER STROTHER IN THE WALTERS ART MUSEUM

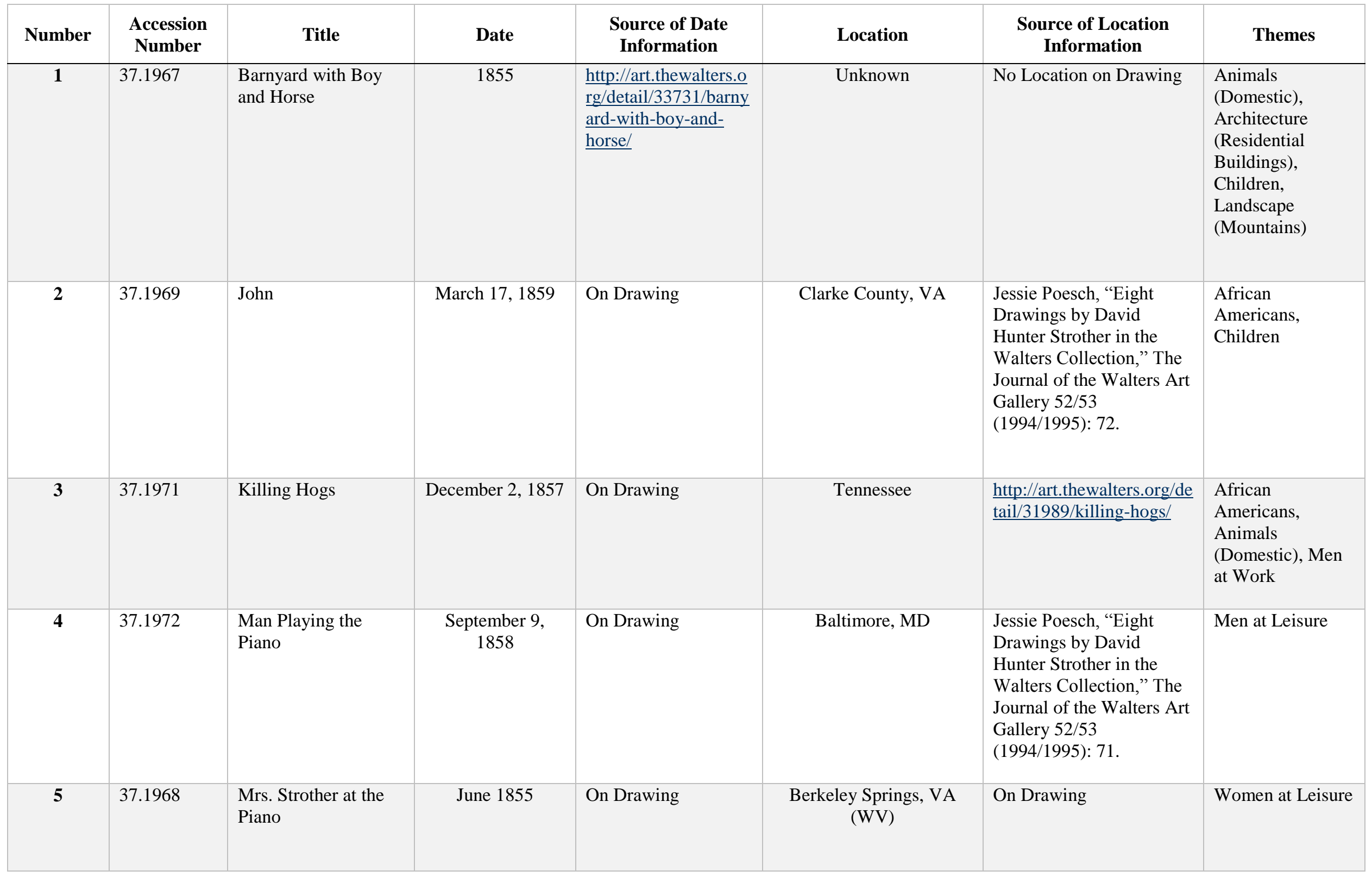




\begin{tabular}{|c|c|c|c|c|c|c|c|}
\hline Number & $\begin{array}{l}\text { Accession } \\
\text { Number }\end{array}$ & Title & Date & $\begin{array}{l}\text { Source of Date } \\
\text { Information }\end{array}$ & Location & $\begin{array}{l}\text { Source of Location } \\
\text { Information }\end{array}$ & Themes \\
\hline 6 & 37.1970 & Obese Man & $\begin{array}{c}\text { September } 10, \\
1858\end{array}$ & On Drawing & Baltimore, MD & $\begin{array}{l}\text { Jessie Poesch, "Eight } \\
\text { Drawings by David } \\
\text { Hunter Strother in the } \\
\text { Walters Collection," The } \\
\text { Journal of the Walters Art } \\
\text { Gallery 52/53 } \\
\text { (1994/1995): } 71 .\end{array}$ & Men \\
\hline 7 & 37.1974 & Three Men & May 6,1859 & On Drawing & Indian Head, MD & $\begin{array}{l}\text { Jessie Poesch, "Eight } \\
\text { Drawings by David } \\
\text { Hunter Strother in the } \\
\text { Walters Collection," The } \\
\text { Journal of the Walters Art } \\
\text { Gallery 52/53 } \\
\text { (1994/1995): } 75 \text {. }\end{array}$ & Men at Leisure \\
\hline 8 & 37.1973 & Three Turkeys & March 15, 1859 & On Drawing & Clarke County, VA & On Drawing & Animals \\
\hline
\end{tabular}


APPENDIX B: ARTWORKS OF DAVID HUNTER STROTHER IN THE WEST VIRGINIA \& REGIONAL HISTORY CENTER'S DIGITAL COLLECTION

\begin{tabular}{|c|c|c|c|c|c|c|c|}
\hline Number & Accession Number & Title & Date & $\begin{array}{l}\text { Source of Date } \\
\text { Information }\end{array}$ & Location & $\begin{array}{l}\text { Source of Location } \\
\text { Information }\end{array}$ & Themes \\
\hline 1 & W1995.030.259 & $\begin{array}{l}\text { 15th..November } 1856 \text { Sam } \\
\text { Bug James River Canal }\end{array}$ & $\begin{array}{l}\text { November } \\
15,1856\end{array}$ & On Drawing & Virginia & $\begin{array}{l}\text { http://www.wvencycloped } \\
\text { ia.org/articles/964 }\end{array}$ & $\begin{array}{l}\text { Men at Work, } \\
\text { Transportation } \\
\text { (Boats) }\end{array}$ \\
\hline 2 & W1995.030.388pg7 & 1845. Winchester. Va & 1845 & On Drawing & Winchester, VA & On Drawing & $\begin{array}{l}\text { Decorative } \\
\text { Arts, Women } \\
\text { at Leisure }\end{array}$ \\
\hline 3 & W1995.030.387pg25a & 1847 & 1847 & On Drawing & Unknown & No Location on Drawing & Men at Leisure \\
\hline 4 & W1995.030.387pg25b & 1847 & 1847 & On Drawing & Unknown & No Location on Drawing & $\begin{array}{l}\text { Decorative } \\
\text { Arts, Men at } \\
\text { Leisure }\end{array}$ \\
\hline 5 & W1995.030.390pg12 & $\begin{array}{l}1850 \text { October } 1848 \text { (Sketches } \\
\text { of children and man sleeping) }\end{array}$ & $\begin{array}{l}1848 \text { and } \\
1850\end{array}$ & On Drawing & Unknown & No Location on Drawing & $\begin{array}{l}\text { African } \\
\text { Americans, } \\
\text { Children, } \\
\text { Decorative } \\
\text { Arts, Men at } \\
\text { Leisure }\end{array}$ \\
\hline 6 & W1995.030.390pg19b & 1852 & 1852 & On Drawing & Unknown & No Location on Drawing & Men at Leisure \\
\hline 7 & W1995.030.390pg20b & 1852 Blackwater Characters & 1852 & On Drawing & $\begin{array}{l}\text { Canaan Valley, VA } \\
\text { (WV) }\end{array}$ & On Drawing & Men \\
\hline 8 & W1995.030.390pg21b & 1852 Blackwater Expedition & 1852 & On Drawing & $\begin{array}{l}\text { Canaan Valley, VA } \\
\text { (WV) }\end{array}$ & On Drawing & $\begin{array}{l}\text { Transportation } \\
\text { (Carriages) }\end{array}$ \\
\hline 9 & W1995.030.390pg25a & $\begin{array}{l}1853 \text { (Various sketches - } \\
\text { woman on horseback, Don } \\
\text { Quixote, P. Pendleton's Owl) }\end{array}$ & 1853 & On Drawing & Unknown & No Location on Drawing & $\begin{array}{l}\text { Animals, } \\
\text { Decorative } \\
\text { Arts, Men at } \\
\text { Leisure, } \\
\text { Women }\end{array}$ \\
\hline
\end{tabular}




\begin{tabular}{|c|c|c|c|c|c|c|c|}
\hline Number & Accession Number & Title & Date & $\begin{array}{l}\text { Source of Date } \\
\text { Information }\end{array}$ & Location & $\begin{array}{l}\text { Source of Location } \\
\text { Information }\end{array}$ & Themes \\
\hline 10 & W1995.030.387pg27a & 1854 & 1854 & On Drawing & Unknown & No Location on Drawing & Children \\
\hline 11 & W1995.030.316 & $\begin{array}{l}1854 \text { Oct } 21 \text { st Mouth of North } \\
\text { Fork - Hardy Co. No } 5 . \\
\text { Arched strata - West Va }\end{array}$ & $\begin{array}{l}\text { October 21, } \\
1854\end{array}$ & On Drawing & $\begin{array}{l}\text { Hardy County, VA } \\
\text { (WV) }\end{array}$ & On Drawing & $\begin{array}{l}\text { Landscape } \\
\text { (Mountains), } \\
\text { Landscape } \\
\text { (Rivers) }\end{array}$ \\
\hline 12 & W1995.030.387pg22a & $\begin{array}{l}\text { 1858 July 23rd (a) - March } \\
\text { 13th } 1859 \text { Clarke Co Va }\end{array}$ & $\begin{array}{l}\text { August 14, } \\
1858\end{array}$ & On Drawing & $\begin{array}{l}\text { Berkeley Springs, VA } \\
\text { (WV) }\end{array}$ & On Drawing & Men at Leisure \\
\hline 13 & W1995.030.229 & 1877 & 1877 & On Drawing & Unknown & No Location on Drawing & Women \\
\hline 14 & W1995.030.267b & $\begin{array}{l}6 \text { portraits Martinsburg } 1874 \\
\text { Shirely - Morgan Co. } 1873 \\
\text { Feb 8th } 1874 \text { Berkeley } \\
\text { Springs } 18581874 \text { January } \\
\text { 26th } 1874\end{array}$ & $\begin{array}{l}1858,1873, \\
\text { and } 1874\end{array}$ & On Drawing & $\begin{array}{l}\text { Berkeley Springs, VA } \\
\text { (WV)/ Martinsburg, } \\
\text { WV/ Morgan County, } \\
\text { WV }\end{array}$ & On Drawing & $\begin{array}{l}\text { Decorative } \\
\text { Arts, Men, } \\
\text { Women }\end{array}$ \\
\hline 15 & W1995.030.339 & $\begin{array}{l}\text { Aaron Armantrout. Sept. } \\
\text { 1860. Mouth of the Gandy- } \\
\text { Randolph Co.. Va. }\end{array}$ & $\begin{array}{l}\text { September, } \\
1860\end{array}$ & On Drawing & $\begin{array}{l}\text { Randolph County, VA } \\
\text { (WV) }\end{array}$ & On Drawing & $\begin{array}{l}\text { Animals } \\
\text { (Domestic), } \\
\text { Men at Work }\end{array}$ \\
\hline 16 & W1995.030.394pg27 & $\begin{array}{l}\text { Aaron C. Stephens on Trial, } \\
\text { Oct. } 26 \text { 1859. Charleston, Va. }\end{array}$ & $\begin{array}{l}\text { October 26, } \\
1859\end{array}$ & On Drawing & $\begin{array}{l}\text { Charles Town, VA } \\
\text { (WV) }\end{array}$ & On Drawing & $\begin{array}{l}\text { Harper's Ferry } \\
\text { Raid Trials, } \\
\text { Men }\end{array}$ \\
\hline 17 & W1995.030.323a & $\begin{array}{l}\text { About Berkeley Springs on } \\
\text { Court day }\end{array}$ & $\begin{array}{l}\text { October } 9 \\
1875\end{array}$ & $\begin{array}{l}\text { WVRHC } \\
\text { Description Page }\end{array}$ & $\begin{array}{l}\text { Berkeley Springs, VA } \\
\text { (WV) }\end{array}$ & On Drawing & $\begin{array}{l}\text { Animals } \\
\text { (Domestic), } \\
\text { Men, Women }\end{array}$ \\
\hline 18 & W1995.030.392pg13 & Accomac April 27th 1887 & $\begin{array}{l}\text { April 27, } \\
1887\end{array}$ & On Drawing & Accomack County, VA & On Drawing & $\begin{array}{l}\text { Men at Work, } \\
\text { Transportation } \\
\text { (Boats) }\end{array}$ \\
\hline
\end{tabular}




\begin{tabular}{|c|c|c|c|c|c|c|c|}
\hline Number & Accession Number & Title & Date & $\begin{array}{l}\text { Source of Date } \\
\text { Information }\end{array}$ & Location & $\begin{array}{l}\text { Source of Location } \\
\text { Information }\end{array}$ & Themes \\
\hline 19 & W1995.030.392pg12 & Accomac April 29th 1887 & $\begin{array}{l}\text { April 29, } \\
1887\end{array}$ & On Drawing & Accomack County, VA & On Drawing & Men \\
\hline 20 & W1995.030.032 & After Raphael & 1875 & On Drawing & Unknown & No Location on Drawing & $\begin{array}{l}\text { Copies of } \\
\text { European } \\
\text { Artwork }\end{array}$ \\
\hline 21 & W1995.030.359 & $\begin{array}{l}\text { Afternoon Drill. Hapers ferry } \\
\text { May 22nd...1861 }\end{array}$ & May 22, 1861 & On Drawing & $\begin{array}{l}\text { Harpers Ferry, VA } \\
\text { (WV) }\end{array}$ & On Drawing & $\begin{array}{l}\text { Landscape } \\
\text { (Mountains), } \\
\text { Men, Military }\end{array}$ \\
\hline 22 & W1995.030.393pg9a & $\begin{array}{l}\text { Alabama River Feb. 8th } 1857 . \\
\text { Mississippi Steamboat }\end{array}$ & $\begin{array}{l}\text { February } 8 \\
\quad 1857\end{array}$ & On Drawing & Alabama, Mississippi & On Drawing & $\begin{array}{l}\text { African } \\
\text { Americans, } \\
\text { Animals } \\
\text { (Domestic), } \\
\text { Children }\end{array}$ \\
\hline 23 & W1995.030.390pg3a & Allgany Virginia - 1844, Nov & $\begin{array}{l}\text { November, } \\
1844\end{array}$ & On Drawing & Allegheny County, VA & On Drawing & $\begin{array}{l}\text { Landscape } \\
\text { (Fences), } \\
\text { Landscape } \\
\text { (Mountains), } \\
\text { Landscape } \\
\text { (Roads), Men, } \\
\text { Women }\end{array}$ \\
\hline 24 & W1995.030.154 & $\begin{array}{l}\text { American Cemetery at } \\
\text { Traxpama near the city of } \\
\text { Mexico outlined. March 18, } \\
\text { 1883. at the } \\
\text { cemetery....completed July 30, } \\
\text { 1887. at Charlestown U.S.A. } \\
\text { (en versa-Greek architecture) }\end{array}$ & $\begin{array}{c}\text { March 18, } \\
1883 \text { and July } \\
30,1887\end{array}$ & On Drawing & Mexico City, Mexico & On Drawing & Cemeteries \\
\hline 25 & W1995.030.096 & $\begin{array}{l}\text { Among the rocks near } \\
\text { Vallombrosa Tuscany }\end{array}$ & 1842 & On Drawing & Vallombrosa, Italy & On Drawing & $\begin{array}{l}\text { Landscape } \\
\text { (Mountains), } \\
\text { Religious } \\
\text { Shrines }\end{array}$ \\
\hline
\end{tabular}




\begin{tabular}{|c|c|c|c|c|c|c|c|}
\hline Number & Accession Number & Title & Date & $\begin{array}{l}\text { Source of Date } \\
\text { Information }\end{array}$ & Location & $\begin{array}{l}\text { Source of Location } \\
\text { Information }\end{array}$ & Themes \\
\hline 26 & W1995.030.266 & Ann Doyne Nov: 30" 1857 & $\begin{array}{l}\text { November } \\
30,1857\end{array}$ & On Drawing & Unknown & No Location on Drawing & Women \\
\hline 27 & W1995.030.390pg10 & $\begin{array}{l}\text { Anny [?] (Various sketches - } \\
\text { baby, horses and wagon, } \\
\text { women crossing stream, } \\
\text { trousers) }\end{array}$ & Unknown & $\begin{array}{l}\text { No Date on } \\
\text { Drawing }\end{array}$ & Unknown & No Location on Drawing & $\begin{array}{l}\text { Animals } \\
\text { (Domestic), } \\
\text { Children, } \\
\text { Landscape } \\
\text { (Fences), } \\
\text { Landscape } \\
\text { (Roads), Men } \\
\text { in Wilderness, } \\
\text { Transportation } \\
\text { (Carriages), } \\
\text { Women in } \\
\text { Wilderness }\end{array}$ \\
\hline 28 & W1995.030.344 & April 13th..1857. Wheeling- & $\begin{array}{l}\text { April 13, } \\
1857\end{array}$ & On Drawing & Wheeling, VA (WV) & On Drawing & $\begin{array}{l}\text { Architecture } \\
\text { (Bridges), } \\
\text { Landscape } \\
\text { (Mountains), } \\
\text { Landscape } \\
\text { (Rivers), Men } \\
\text { at Work, } \\
\text { Transportation } \\
\text { (Boats) }\end{array}$ \\
\hline 29 & W1995.030.036 & April 3rd 1877 & April 3, 1877 & On Drawing & Unknown & No Location on Drawing & $\begin{array}{l}\text { Architecture, } \\
\text { Cemeteries, } \\
\text { Landscape }\end{array}$ \\
\hline 30 & W1995.030.392pg8b & $\begin{array}{l}\text { Arlington. Northhampton Co } \\
\text { Va May } 61887\end{array}$ & May 6,1887 & On Drawing & Arlington, VA & On Drawing & $\begin{array}{l}\text { Architecture } \\
\text { (Residential } \\
\text { Buildings) }\end{array}$ \\
\hline
\end{tabular}




\begin{tabular}{|c|c|c|c|c|c|c|c|}
\hline Number & Accession Number & Title & Date & $\begin{array}{l}\text { Source of Date } \\
\text { Information }\end{array}$ & Location & $\begin{array}{l}\text { Source of Location } \\
\text { Information }\end{array}$ & Themes \\
\hline 31 & W1995.030.016 & Artists Excursion & 1858 & On Drawing & Unknown & No Location on Drawing & Men \\
\hline 32 & W1995.030.393pg7b & $\begin{array}{l}\text { Artists Excursion } 1859 \text { The } \\
\text { Heir of Genius }\end{array}$ & 1859 & On Drawing & Unknown & No Location on Drawing & Men at Leisure \\
\hline 33 & W1995.030.393pg19b & $\begin{array}{l}\text { Artists Excursion Balt \& Ohio } \\
\text { R.R. }\end{array}$ & Unknown & $\begin{array}{l}\text { No Date on } \\
\text { Drawing }\end{array}$ & Unknown & No Location on Drawing & Men \\
\hline 34 & W1995.030.393pg8b & $\begin{array}{l}\text { Artists Excursion Balt \& Ohio } \\
\text { R.R. West Va. }\end{array}$ & Unknown & $\begin{array}{l}\text { No Date on } \\
\text { Drawing }\end{array}$ & West Virginia & On Drawing & Animals \\
\hline 35 & W1995.030.386pg1a & Ascending the ridges. & Unknown & $\begin{array}{l}\text { No Date on } \\
\text { Drawing }\end{array}$ & $\begin{array}{c}\text { Canaan Valley, VA } \\
\text { (WV) }\end{array}$ & $\begin{array}{c}\text { Strother, "The Virginian } \\
\text { Canaan," Harpers New } \\
\text { Monthly Magazine, } \\
\text { December } 1853,25 .\end{array}$ & $\begin{array}{l}\text { Animals } \\
\text { (Domestic), } \\
\text { Landscape } \\
\text { (Mountains), } \\
\text { Men in } \\
\text { Wilderness }\end{array}$ \\
\hline 36 & W1995.030.337 & $\begin{array}{l}\text { Ascenet (sic) of Otter Peak. } \\
1853\end{array}$ & 1853 & On Drawing & Botetourt County, VA & See Number 166 & $\begin{array}{l}\text { African } \\
\text { Americans, } \\
\text { Landscape } \\
\text { (Mountains), } \\
\text { Men in } \\
\text { Wilderness, } \\
\text { Women in } \\
\text { Wilderness }\end{array}$ \\
\hline 37 & W1995.030.310 & $\begin{array}{l}\text { Asylum for the deaf mute \& } \\
\text { blind Staunton. Oct. } 18.1853 \text {. }\end{array}$ & $\begin{array}{l}\text { October } 18 \\
1853\end{array}$ & On Drawing & Staunton, VA & On Drawing & $\begin{array}{l}\text { Architecture } \\
\text { (Institutional } \\
\text { Buildings), } \\
\text { Landscape } \\
\text { (Mountains) }\end{array}$ \\
\hline
\end{tabular}




\begin{tabular}{|c|c|c|c|c|c|c|c|}
\hline Number & Accession Number & Title & Date & $\begin{array}{l}\text { Source of Date } \\
\text { Information }\end{array}$ & Location & $\begin{array}{l}\text { Source of Location } \\
\text { Information }\end{array}$ & Themes \\
\hline 38 & W1995.030.171 & $\begin{array}{l}\text { Asylum for the insane-Raleigh } \\
\text { N.C. April 22, } 1856\end{array}$ & $\begin{array}{l}\text { April 22, } \\
1856\end{array}$ & On Drawing & Raleigh, NC & On Drawing & $\begin{array}{l}\text { Architecture } \\
\text { (Institutional } \\
\text { Buildings), } \\
\text { Architecture } \\
\text { (Residential } \\
\text { Buildings), } \\
\text { Landscape, } \\
\text { Transportation } \\
\text { (Railroads) }\end{array}$ \\
\hline 39 & W1995.030.308 & $\begin{array}{l}\text { Asylum for the Insane- } \\
\text { Staunton Augusta Co Virginia }\end{array}$ & $\begin{array}{l}\text { March 1, } \\
1874\end{array}$ & $\begin{array}{c}\text { WVRHC } \\
\text { Description Page }\end{array}$ & Staunton, VA & On Drawing & $\begin{array}{l}\text { Architecture } \\
\text { (Institutional } \\
\text { Buildings), } \\
\text { Landscape } \\
\text { (Mountains) }\end{array}$ \\
\hline 40 & W1995.030.330 & $\begin{array}{l}\text { At Bakers-No } 37 \text { Miles above } \\
\text { Moorfield Hardy Co-West } \\
\text { Va.. Oct. 7th..1854 (En verso) } \\
\text { Moorfield \& Petersburg }\end{array}$ & $\begin{array}{l}\text { October } 7 \\
1854\end{array}$ & On Drawing & $\begin{array}{l}\text { Hardy County, VA } \\
\text { (WV) }\end{array}$ & On Drawing & $\begin{array}{l}\text { Landscape } \\
\text { (Mountains) }\end{array}$ \\
\hline 41 & W1995.030.387pg26c & $\begin{array}{l}\text { At Hancock Depot Aug 1st } \\
1857\end{array}$ & $\begin{array}{l}\text { August } 1 \\
1857\end{array}$ & On Drawing & Hancock, MD & On Drawing & $\begin{array}{l}\text { Animals } \\
\text { (Domestic), } \\
\text { Landscape } \\
\text { (Fences), } \\
\text { Transportation } \\
\text { (Carriages) }\end{array}$ \\
\hline 42 & W1995.030.064 & At Manasas Junction & $\begin{array}{l}\text { August 22, } \\
\quad 1869\end{array}$ & On Drawing & Manassas, VA & On Drawing & $\begin{array}{l}\text { Architecture, } \\
\text { Landscape, } \\
\text { Men at Leisure, } \\
\text { Military }\end{array}$ \\
\hline
\end{tabular}




\begin{tabular}{|c|c|c|c|c|c|c|c|}
\hline Number & Accession Number & Title & Date & $\begin{array}{l}\text { Source of Date } \\
\text { Information }\end{array}$ & Location & $\begin{array}{l}\text { Source of Location } \\
\text { Information }\end{array}$ & Themes \\
\hline 43 & W1995.030.395pg3 & $\begin{array}{l}\text { Aug 1. 1873. Camp Williams } \\
\text { Wilsons Ranche Rocky } \\
\text { Mountains }\end{array}$ & $\begin{array}{c}\text { August } 1, \\
1873\end{array}$ & On Drawing & Rocky Mountains & On Drawing & 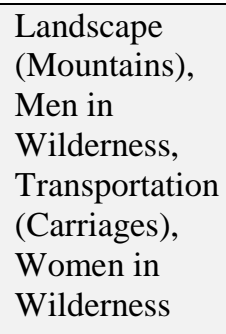 \\
\hline 44 & W1995.030.387pg5 & $\begin{array}{l}\text { August, } 1846 \text { Berkeley } \\
\text { Springs, Va August } 1846 \\
\text { (horse) }\end{array}$ & August 1846 & On Drawing & $\begin{array}{c}\text { Berkeley Springs, VA } \\
\text { (WV) }\end{array}$ & On Drawing & $\begin{array}{l}\text { Animals } \\
\text { (Domestic), } \\
\text { Decorative } \\
\text { Arts, Men at } \\
\text { Leisure }\end{array}$ \\
\hline 45 & W1995.030.395pg8a & B \& O. R.R. June 26th 1874. & $\begin{array}{c}\text { June } 26,1874 \\
\text { and January } \\
30,1876\end{array}$ & On Drawing & Unknown & No Location on Drawing & Men at Leisure \\
\hline 46 & W1995.030.393pg14a & Back Ashby Clarke Co. 1859. & 1859 & On Drawing & Clarke County, VA & On Drawing & Men at Leisure \\
\hline 47 & W1995.030.027 & Baltimore & $\begin{array}{c}\text { March 4, } \\
1860\end{array}$ & On Drawing & Baltimore, MD & On Drawing & $\begin{array}{l}\text { Decorative } \\
\text { Arts }\end{array}$ \\
\hline 48 & W1995.030.388pg18 & Baltimore 1845. & 1845 & On Drawing & Baltimore, MD & On Drawing & $\begin{array}{l}\text { Decorative } \\
\text { Arts, Men at } \\
\text { Leisure }\end{array}$ \\
\hline 49 & W1995.030.388pg2 & Baltimore 1845. & 1845 & On Drawing & Baltimore, MD & On Drawing & Men \\
\hline
\end{tabular}




\begin{tabular}{|c|c|c|c|c|c|c|c|}
\hline Number & Accession Number & Title & Date & $\begin{array}{l}\text { Source of Date } \\
\text { Information }\end{array}$ & Location & $\begin{array}{l}\text { Source of Location } \\
\text { Information }\end{array}$ & Themes \\
\hline 50 & W1995.030.387pg10 & $\begin{array}{l}\text { Banks of Potomac. Sir John's } \\
\text { Run. October } 24,1848\end{array}$ & $\begin{array}{l}\text { October } 24 \\
1848\end{array}$ & On Drawing & $\begin{array}{l}\text { Berkeley Springs, VA } \\
\text { (WV) }\end{array}$ & $\begin{array}{c}\text { Cecil Eby, "Porte } \\
\text { Crayon": The Life of } \\
\text { David Hunter Strother, } \\
\text { (Chapel Hill, NC: } \\
\text { University of North } \\
\text { Carolina Press, 1960), } 56 .\end{array}$ & $\begin{array}{l}\text { Landscape, } \\
\text { Men in } \\
\text { Wilderness }\end{array}$ \\
\hline 51 & W1995.030.138 & Banks of the Mississippi & Unknown & $\begin{array}{l}\text { Date on Drawing } \\
\text { Unclear }\end{array}$ & Mississippi & On Drawing & $\begin{array}{l}\text { African } \\
\text { Americans, } \\
\text { Architecture, } \\
\text { Children, } \\
\text { Landscape } \\
\text { (Rivers), Men } \\
\text { at Leisure, } \\
\text { Women at } \\
\text { Leisure }\end{array}$ \\
\hline 52 & W1995.030.390pg8 & $\begin{array}{l}\text { Banks of the Potomac. Octo } \\
\text { 1848. near Sir John's Theo. } \\
\text { Kennedy. }\end{array}$ & October 1848 & On Drawing & $\begin{array}{l}\text { Berkeley Springs, VA } \\
\text { (WV) }\end{array}$ & $\begin{array}{l}\text { Cecil Eby, "Porte } \\
\text { Crayon": The Life of } \\
\text { David Hunter Strother, } \\
\text { (Chapel Hill, NC: } \\
\text { University of North } \\
\text { Carolina Press, 1960), } 56 .\end{array}$ & Landscape \\
\hline 53 & W1995.030.392pg6 & $\begin{array}{l}\text { Barber's Shop at Head of } \\
\text { Neck April 30th } 1887\end{array}$ & $\begin{array}{l}\text { April 30, } \\
1887\end{array}$ & On Drawing & Unknown & No Location on Drawing & $\begin{array}{l}\text { African } \\
\text { Americans, } \\
\text { Men at Work }\end{array}$ \\
\hline 54 & W1995.030.102 & $\begin{array}{l}\text { Base \& servile imitation of } \\
\text { Waugh }\end{array}$ & Unknown & $\begin{array}{l}\text { No Date on } \\
\text { Drawing }\end{array}$ & Unknown & No Location on Drawing & Architecture \\
\hline
\end{tabular}




\begin{tabular}{|c|c|c|c|c|c|c|c|}
\hline Number & Accession Number & Title & Date & $\begin{array}{l}\text { Source of Date } \\
\text { Information }\end{array}$ & Location & $\begin{array}{l}\text { Source of Location } \\
\text { Information }\end{array}$ & Themes \\
\hline 55 & W1995.030.309 & $\begin{array}{l}\text { Bath Alum Springs. Bath Co: } \\
\text { Virginia October 20th.. } 1853\end{array}$ & $\begin{array}{l}\text { October 20, } \\
1853\end{array}$ & On Drawing & Bath County, VA & On Drawing & $\begin{array}{l}\text { Architecture } \\
\text { (Institutional } \\
\text { Buildings), } \\
\text { Landscape } \\
\text { (Mountains), } \\
\text { Men, Women }\end{array}$ \\
\hline 56 & W2002.050 & Bath Keeper & 1848 & $\begin{array}{c}\text { John A. } \\
\text { Cuthbert, "Early } \\
\text { Art and Artists in } \\
\text { West Virginia," } \\
\text { (Morgantown, } \\
\text { WV: West } \\
\text { Virginia } \\
\text { University Press, } \\
\text { 2000), 24. }\end{array}$ & $\begin{array}{l}\text { Berkeley Springs, VA } \\
\text { (WV) }\end{array}$ & $\frac{\frac{\text { http://www.berkeleysprin }}{\text { gs.com/walkingtour/10inn }}}{\text { spa.html }}$ & $\begin{array}{l}\text { Animals } \\
\text { (Domestic), } \\
\text { Men at Work }\end{array}$ \\
\hline 57 & W1995.030.136 & $\begin{array}{l}\text { Baton Rouge Cemetry [sic.] } \\
\text { Bastion }\end{array}$ & $\begin{array}{l}\text { March 11, } \\
\quad 1863\end{array}$ & On Drawing & Baton Rouge, LA & On Drawing & $\begin{array}{l}\text { Cemeteries, } \\
\text { Landscape }\end{array}$ \\
\hline 58 & W1995.030.125 & Baton Rouge March 9th 1863 & $\begin{array}{l}\text { March 9, } \\
1863\end{array}$ & On Drawing & Baton Rouge, LA & On Drawing & $\begin{array}{l}\text { Architecture } \\
\text { (Towns), } \\
\text { Landscape } \\
\text { (Rivers), Men } \\
\text { at Work, } \\
\text { Transportation } \\
\text { (Boats), } \\
\text { Transportation } \\
\text { (Carriages) }\end{array}$ \\
\hline 59 & W1995.030.393pg28a & $\begin{array}{l}\text { Bayou Boeuf - Louisiana - } \\
\text { April } 1857 .\end{array}$ & April 1857 & On Drawing & Louisiana & On Drawing & $\begin{array}{l}\text { Architecture, } \\
\text { Landscape } \\
\text { (River), } \\
\text { Transportation } \\
\text { (Boats) }\end{array}$ \\
\hline
\end{tabular}




\begin{tabular}{|c|c|c|c|c|c|c|c|}
\hline Number & Accession Number & Title & Date & $\begin{array}{l}\text { Source of Date } \\
\text { Information }\end{array}$ & Location & $\begin{array}{l}\text { Source of Location } \\
\text { Information }\end{array}$ & Themes \\
\hline 60 & W1995.030.142 & $\begin{array}{l}\text { Bayou Boeuf Station-New } \\
\text { Olreans \& Opelovsas R.R. } \\
\text { Near Berwicks Bay }\end{array}$ & Unknown & $\begin{array}{l}\text { No Date on } \\
\text { Drawing }\end{array}$ & Louisiana & On Drawing & $\begin{array}{l}\text { Architecture, } \\
\text { Landscape } \\
\text { (Rivers) }\end{array}$ \\
\hline 61 & W1995.030.134 & Bayou St. John. March2 & $\begin{array}{c}\text { December } 9 \\
1856\end{array}$ & $\begin{array}{c}\text { WVRHC } \\
\text { Description Page }\end{array}$ & Louisiana & On Drawing & $\begin{array}{l}\text { Landscape } \\
\text { (Rivers), Men } \\
\text { at Work, } \\
\text { Transportation } \\
\text { (Boats) }\end{array}$ \\
\hline 62 & W1995.030.132 & Bayou Tesche Louisiana & $\begin{array}{c}\text { March 8, } \\
1857\end{array}$ & On Drawing & Louisiana & On Drawing & $\begin{array}{l}\text { Architecture, } \\
\text { Landscape }\end{array}$ \\
\hline 63 & W1995.030.008 & Bear's Den & May 12,1855 & On Drawing & Unknown & No Location on Drawing & Landscape \\
\hline 64 & W1995.030.169 & $\begin{array}{l}\text { Belevedere Fishing-Seaward } \\
\text { Boat }\end{array}$ & April 8, 1856 & On Drawing & Belvedere, NC & $\begin{array}{c}\text { Cecil Eby, "Porte } \\
\text { Crayon": The Life of } \\
\text { David Hunter Strother, } \\
\text { (Chapel Hill, NC: } \\
\text { University of North } \\
\text { Carolina Press, 1960), } 91 .\end{array}$ & $\begin{array}{l}\text { African } \\
\text { Americans, } \\
\text { Animals } \\
\text { (Domestic), } \\
\text { Landscape } \\
\text { (Lakes), Men } \\
\text { at Work, } \\
\text { Transportation } \\
\text { (Boats) }\end{array}$ \\
\hline 65 & W1995.030.095 & Below Vallombrosa & June 1842 & On Drawing & Vallombrosa, Italy & On Drawing & $\begin{array}{l}\text { Landscape } \\
\text { (Mountains), } \\
\text { Landscape } \\
\text { (Rivers) }\end{array}$ \\
\hline 66 & W1995.030.099 & $\begin{array}{l}\text { Below Vallombrosa } 1842 . \\
\text { Ponte Sieve June 1st } 1842\end{array}$ & 1842 & On Drawing & Vallombrosa, Italy & On Drawing & $\begin{array}{l}\text { Animals } \\
\text { (Domestic), } \\
\text { Landscape } \\
\text { (Mountains), } \\
\text { Landscape } \\
\text { (Rivers), Men } \\
\text { in Wilderness }\end{array}$ \\
\hline
\end{tabular}




\begin{tabular}{|c|c|c|c|c|c|c|c|}
\hline Number & Accession Number & Title & Date & $\begin{array}{l}\text { Source of Date } \\
\text { Information }\end{array}$ & Location & $\begin{array}{l}\text { Source of Location } \\
\text { Information }\end{array}$ & Themes \\
\hline 67 & W1995.030.260 & Berkeley Springs & $\begin{array}{l}\text { September } \\
30,1876\end{array}$ & On Drawing & $\begin{array}{c}\text { Berkeley Springs, VA } \\
\text { (WV) }\end{array}$ & On Drawing & Children \\
\hline 68 & W1995.030.387pg28a & Berkeley Springs & $\begin{array}{c}\text { December } 1 \\
1858\end{array}$ & $\begin{array}{c}\text { WVRHC } \\
\text { Description Page }\end{array}$ & $\begin{array}{c}\text { Berkeley Springs, VA } \\
\text { (WV) }\end{array}$ & On Drawing & $\begin{array}{l}\text { Animals } \\
\text { (Domestic) }\end{array}$ \\
\hline 69 & W1995.030.387pg28b & Berkeley Springs & August 1846 & $\begin{array}{c}\text { WVRHC } \\
\text { Description Page }\end{array}$ & $\begin{array}{l}\text { Berkeley Springs, VA } \\
\text { (WV) }\end{array}$ & On Drawing & $\begin{array}{l}\text { Animals } \\
\text { (Domestic) }\end{array}$ \\
\hline 70 & W1995.030.394pg20a & Berkeley Springs & $\begin{array}{c}\text { November } \\
16,1872\end{array}$ & On Drawing & $\begin{array}{l}\text { Berkeley Springs, VA } \\
\text { (WV) }\end{array}$ & On Drawing & $\begin{array}{l}\text { Animals } \\
\text { (Domestic), } \\
\text { Men }\end{array}$ \\
\hline 71 & W1995.030.390pg18b & $\begin{array}{l}\text { Berkeley Springs Commenced } \\
\text { 1853. Completed Dec. 2nd } \\
\text { 187 }\end{array}$ & $\begin{array}{c}1853 / \\
\text { December 2, } \\
1873\end{array}$ & On Drawing & $\begin{array}{l}\text { Berkeley Springs, VA } \\
\text { (WV) }\end{array}$ & On Drawing & $\begin{array}{l}\text { African } \\
\text { Americans, } \\
\text { Women }\end{array}$ \\
\hline 72 & W1995.030.279 & $\begin{array}{l}\text { Berkeley Springs Mrs. } \\
\text { Waterman }\end{array}$ & $\begin{array}{c}\text { September 9, } \\
1876\end{array}$ & On Drawing & $\begin{array}{c}\text { Berkeley Springs, VA } \\
\text { (WV) }\end{array}$ & On Drawing & Women \\
\hline 73 & W1995.030.270 & $\begin{array}{l}\text { Berkeley Springs (boy with } \\
\text { fish) }\end{array}$ & $\begin{array}{c}\text { December } 11 \\
1873\end{array}$ & On Drawing & $\begin{array}{l}\text { Berkeley Springs, VA } \\
\text { (WV) }\end{array}$ & On Drawing & $\begin{array}{l}\text { Animals } \\
\text { (Wild), } \\
\text { Children }\end{array}$ \\
\hline 74 & W1995.030.368 & $\begin{array}{l}\text { Berkeley Springs, Va., } 1858 \\
\text { (Gentleman with cane) }\end{array}$ & 1858 & On Drawing & $\begin{array}{c}\text { Berkeley Springs, VA } \\
\text { (WV) }\end{array}$ & On Drawing & Men \\
\hline 75 & W1995.030.388pg12 & Berkeley Springs, 1846 & 1846 & On Drawing & $\begin{array}{c}\text { Berkeley Springs, VA } \\
\text { (WV) }\end{array}$ & On Drawing & $\begin{array}{l}\text { Animals } \\
\text { (Domestic), } \\
\text { Children }\end{array}$ \\
\hline 76 & W1995.030.388pg13 & Berkeley Springs, 1846 & 1846 & On Drawing & $\begin{array}{l}\text { Berkeley Springs, VA } \\
\text { (WV) }\end{array}$ & On Drawing & $\begin{array}{l}\text { Animals } \\
\text { (Domestic), } \\
\text { Children }\end{array}$ \\
\hline 77 & W1995.030.393pg15a & Berkeley Springs, 1858. & 1858 & On Drawing & $\begin{array}{c}\text { Berkeley Springs, VA } \\
\text { (WV) }\end{array}$ & On Drawing & $\begin{array}{l}\text { Men at Leisure, } \\
\text { Women at } \\
\text { Leisure }\end{array}$ \\
\hline
\end{tabular}




\begin{tabular}{|c|c|c|c|c|c|c|c|}
\hline Number & Accession Number & Title & Date & $\begin{array}{l}\text { Source of Date } \\
\text { Information }\end{array}$ & Location & $\begin{array}{l}\text { Source of Location } \\
\text { Information }\end{array}$ & Themes \\
\hline 78 & W1995.030.394pg16b & $\begin{array}{l}\text { Berkeley Springs, } 1870- \\
\text { Berkeley Springs, Nov. 16th, } \\
1872\end{array}$ & $\begin{array}{l}\text { 1870/Novem } \\
\text { ber } 16,1872\end{array}$ & On Drawing & $\begin{array}{l}\text { Berkeley Springs, VA } \\
\text { (WV) }\end{array}$ & On Drawing & $\begin{array}{l}\text { Animals } \\
\text { (Domestic), } \\
\text { Men }\end{array}$ \\
\hline 79 & W1995.030.394pg32b & $\begin{array}{l}\text { Berkeley Springs, 1870. Just } \\
\text { from Hancock }\end{array}$ & 1870 & On Drawing & $\begin{array}{l}\text { Berkeley Springs, VA } \\
\text { (WV) }\end{array}$ & On Drawing & Men \\
\hline 80 & W1995.030.387pg6a & $\begin{array}{l}\text { Berkeley Springs, April 11th, } \\
1847 .\end{array}$ & $\begin{array}{l}\text { April 11, } \\
1847\end{array}$ & On Drawing & $\begin{array}{l}\text { Berkeley Springs, VA } \\
\text { (WV) }\end{array}$ & On Drawing & $\begin{array}{l}\text { Decorative } \\
\text { Arts, Men }\end{array}$ \\
\hline 81 & W1995.030.366 & $\begin{array}{l}\text { Berkeley Springs, April 20, } \\
1872\end{array}$ & $\begin{array}{l}\text { April 20, } \\
1872\end{array}$ & On Drawing & $\begin{array}{l}\text { Berkeley Springs, VA } \\
\text { (WV) }\end{array}$ & On Drawing & Men \\
\hline 82 & W1995.030.387pg22c & $\begin{array}{l}\text { Berkeley Springs, Aug 14th, } \\
1858\end{array}$ & $\begin{array}{l}\text { August 14, } \\
\quad 1858\end{array}$ & On Drawing & $\begin{array}{l}\text { Berkeley Springs, VA } \\
\text { (WV) }\end{array}$ & On Drawing & Men at Leisure \\
\hline 83 & W1995.030.387pg19 & $\begin{array}{l}\text { Berkeley Springs, Aug 16th, } \\
\text { 1858 Sarah Stange Berkeley } \\
\text { Springs, Aug 25th, } 1858\end{array}$ & $\begin{array}{l}\text { August 16, } \\
\text { 1858/ August } \\
25,1858\end{array}$ & On Drawing & $\begin{array}{l}\text { Berkeley Springs, VA } \\
\text { (WV) }\end{array}$ & On Drawing & $\begin{array}{l}\text { African } \\
\text { Americans, } \\
\text { Men at Leisure, } \\
\text { Women at } \\
\text { Work }\end{array}$ \\
\hline 84 & W1995.030.325 & $\begin{array}{l}\text { Berkeley Springs, Aug. 16, } \\
1859\end{array}$ & $\begin{array}{l}\text { August } 16 \\
1859\end{array}$ & On Drawing & $\begin{array}{l}\text { Berkeley Springs, VA } \\
\text { (WV) }\end{array}$ & On Drawing & Men at Leisure \\
\hline 85 & W1995.030.387pg46 & $\begin{array}{l}\text { Berkeley Springs, Aug. 5th, } \\
1858 .\end{array}$ & $\begin{array}{l}\text { August } 5 \text {, } \\
1858\end{array}$ & $\begin{array}{c}\text { WVRHC } \\
\text { Description Page }\end{array}$ & $\begin{array}{l}\text { Berkeley Springs, VA } \\
\text { (WV) }\end{array}$ & On Drawing & Women \\
\hline 86 & W1995.030.387pg52 & $\begin{array}{l}\text { Berkeley Springs, Aug: 4th, } \\
1858\end{array}$ & $\begin{array}{l}\text { August } 4, \\
1858\end{array}$ & On Drawing & $\begin{array}{l}\text { Berkeley Springs, VA } \\
\text { (WV) }\end{array}$ & On Drawing & $\begin{array}{l}\text { Women at } \\
\text { Work }\end{array}$ \\
\hline 87 & W1995.030.395pg5 & $\begin{array}{l}\text { Berkeley Springs, December } \\
\text { 2nd } 1873 .\end{array}$ & $\begin{array}{l}\text { December 2, } \\
\quad 1873\end{array}$ & On Drawing & $\begin{array}{l}\text { Berkeley Springs, VA } \\
\text { (WV) }\end{array}$ & On Drawing & $\begin{array}{l}\text { Children, } \\
\text { Decorative } \\
\text { Arts }\end{array}$ \\
\hline 88 & W1995.030.395pg4 & $\begin{array}{l}\text { Berkeley Springs, Nov 29th, } \\
1873 .\end{array}$ & $\begin{array}{l}\text { November } \\
29,1873\end{array}$ & On Drawing & $\begin{array}{l}\text { Berkeley Springs, VA } \\
\text { (WV) }\end{array}$ & On Drawing & $\begin{array}{l}\text { Children, } \\
\text { Decorative } \\
\text { Arts }\end{array}$ \\
\hline
\end{tabular}




\begin{tabular}{|c|c|c|c|c|c|c|c|}
\hline Number & Accession Number & Title & Date & $\begin{array}{l}\text { Source of Date } \\
\text { Information }\end{array}$ & Location & $\begin{array}{l}\text { Source of Location } \\
\text { Information }\end{array}$ & Themes \\
\hline 89 & W1995.030.394pg & $\begin{array}{l}\text { Berkeley Springs, November } \\
\text { 21, } 1872 \text { Eager Nefo }\end{array}$ & $\begin{array}{l}\text { November } \\
21,1872\end{array}$ & On Drawing & $\begin{array}{c}\text { Berkeley Springs, VA } \\
\text { (WV) }\end{array}$ & On Drawing & $\begin{array}{l}\text { Animals } \\
\text { (Domestic) }\end{array}$ \\
\hline 90 & W1995.030.326 & $\begin{array}{l}\text { Berkeley Springs, October } \\
\text { 13th, 1887. Old Morgan } \\
\text { County }\end{array}$ & $\begin{array}{l}\text { October } 13 \\
1887\end{array}$ & On Drawing & $\begin{array}{l}\text { Berkeley Springs, VA } \\
\text { (WV) }\end{array}$ & On Drawing & $\begin{array}{l}\text { Animals } \\
\text { (Domestic), } \\
\text { Men }\end{array}$ \\
\hline 91 & W1995.030.395pg8b & $\begin{array}{l}\text { Berkeley Springs, October 9th, } \\
1875 .\end{array}$ & $\begin{array}{l}\text { April } 2,1875 / \\
\text { October } 9 \\
\quad 1875\end{array}$ & On Drawing & $\begin{array}{l}\text { Charles Town, VA } \\
\text { (WV)/ Berkeley } \\
\text { Springs, VA (WV) }\end{array}$ & On Drawing & Children, Men \\
\hline 92 & W1995.030.395pg2 & $\begin{array}{l}\text { Berkeley Springs, October, } \\
1872 .\end{array}$ & October 1872 & On Drawing & $\begin{array}{c}\text { Berkeley Springs, VA } \\
\text { (WV) }\end{array}$ & On Drawing & Women \\
\hline 93 & W1995.030.242a & $\begin{array}{l}\text { Berkeley Springs, Sept 11, } \\
1876\end{array}$ & $\begin{array}{l}\text { September } \\
11,1876\end{array}$ & On Drawing & $\begin{array}{c}\text { Berkeley Springs, VA } \\
\text { (WV) }\end{array}$ & On Drawing & Men \\
\hline 94 & W1995.030.395pg13 & $\begin{array}{l}\text { Berkeley Springs, Sept 30th, } \\
1872\end{array}$ & $\begin{array}{l}\text { September } \\
30,1872\end{array}$ & On Drawing & $\begin{array}{l}\text { Berkeley Springs, VA } \\
\text { (WV) }\end{array}$ & On Drawing & Landscape \\
\hline 95 & W1995.030.237a & $\begin{array}{l}\text { Berkeley Springs, Sept: } 30 \text {, } \\
1876\end{array}$ & $\begin{array}{l}\text { September } \\
30,1876\end{array}$ & On Drawing & $\begin{array}{c}\text { Berkeley Springs, VA } \\
\text { (WV) }\end{array}$ & On Drawing & Children \\
\hline 96 & W1995.030.237b & $\begin{array}{l}\text { Berkeley Springs, Sept: 30, } \\
1876\end{array}$ & $\begin{array}{l}\text { September } \\
30,1876\end{array}$ & On Drawing & $\begin{array}{l}\text { Berkeley Springs, VA } \\
\text { (WV) }\end{array}$ & On Drawing & Children \\
\hline 97 & W1995.030.276 & $\begin{array}{l}\text { Berkeley Springs, September } \\
(?), 1871\end{array}$ & $\begin{array}{l}\text { September } \\
1871\end{array}$ & On Drawing & $\begin{array}{c}\text { Berkeley Springs, VA } \\
\text { (WV) }\end{array}$ & On Drawing & $\begin{array}{l}\text { Decorative } \\
\text { Arts, Men }\end{array}$ \\
\hline 98 & W1995.030.391pg9 & $\begin{array}{l}\text { Berkeley Springs, September } \\
\text { 3rd, } 1876 .\end{array}$ & $\begin{array}{l}\text { September 3, } \\
1876\end{array}$ & On Drawing & $\begin{array}{c}\text { Berkeley Springs, VA } \\
\text { (WV) }\end{array}$ & On Drawing & Men at work \\
\hline 99 & W1995.030.274 & Berleley Springs Sept. 21871 & $\begin{array}{l}\text { September 2, } \\
1871\end{array}$ & On Drawing & $\begin{array}{c}\text { Berkeley Springs, VA } \\
\text { (WV) }\end{array}$ & On Drawing & Men \\
\hline
\end{tabular}




\begin{tabular}{|c|c|c|c|c|c|c|c|}
\hline Number & Accession Number & Title & Date & $\begin{array}{l}\text { Source of Date } \\
\text { Information }\end{array}$ & Location & $\begin{array}{l}\text { Source of Location } \\
\text { Information }\end{array}$ & Themes \\
\hline 100 & W1995.030.240 & Betsey Swest April 11th 1856 & $\begin{array}{l}\text { April 11, } \\
\quad 1856\end{array}$ & On Drawing & Albemarle Sound, NC & See Number 101 & $\begin{array}{l}\text { African } \\
\text { Americans, } \\
\text { Women }\end{array}$ \\
\hline 101 & W1995.030.170 & $\begin{array}{l}\text { Betsey } \\
\text { Swest/Winslow/Albermenle } \\
\text { Sound, NC }\end{array}$ & April 1856 & On Drawing & Albemarle Sound, NC & On Drawing & $\begin{array}{l}\text { African } \\
\text { Americans, } \\
\text { Animals } \\
\text { (Wild), Men, } \\
\text { Women at } \\
\text { Leisure }\end{array}$ \\
\hline 102 & W1995.030.393pg22b & $\begin{array}{l}\text { Big Scholar. } 1874 \\
\text { Charlestown. West Va. }\end{array}$ & 1874 & On Drawing & $\begin{array}{c}\text { Charles Town, VA } \\
\text { (WV) }\end{array}$ & On Drawing & $\begin{array}{l}\text { African } \\
\text { Americans, } \\
\text { Children, Men }\end{array}$ \\
\hline 103 & W1995.030.028 & Bill Hooper's Saloon & $\begin{array}{c}\text { December } 18 \\
1860\end{array}$ & On Drawing & Unknown & No Location on Drawing & Men \\
\hline 104 & W1995.030.391pg13 & $\begin{array}{l}\text { Bill Napper Marinsburg Va. } \\
1845 .\end{array}$ & 1845 & On Drawing & Martinsburg, VA (WV) & On Drawing & $\begin{array}{l}\text { African } \\
\text { Americans, } \\
\text { Men }\end{array}$ \\
\hline 105 & W1995.030.387pg49 & $\begin{array}{l}\text { Billy Hughes. Passenger Car } \\
\text { Inspector since } 1834 \text {. Balt \& } \\
\text { Ohio R. Road. Oct. 26th } 1858 \text {. }\end{array}$ & $\begin{array}{l}\text { October } 26 \\
1858\end{array}$ & On Drawing & Unknown & No Location on Drawing & Men \\
\hline 106 & W1995.030.056 & $\begin{array}{l}\text { Biting Horse Camp at } \\
\text { Damstown, Montgomery Co. } \\
\text { Md. }\end{array}$ & $\begin{array}{l}\text { August } 30, \\
1861\end{array}$ & On Drawing & $\begin{array}{c}\text { Montgomery County, } \\
\text { MD }\end{array}$ & On Drawing & $\begin{array}{l}\text { Animals } \\
\text { (Domestic) }\end{array}$ \\
\hline 107 & W1995.030.375 & $\begin{array}{l}\text { Bivouac (Virginia Canaan) } \\
\text { June } 1852\end{array}$ & June 1852 & On Drawing & $\begin{array}{c}\text { Canaan Valley, VA } \\
\text { (WV) }\end{array}$ & On Drawing & $\begin{array}{l}\text { Men in } \\
\text { Wilderness }\end{array}$ \\
\hline 108 & W1995.030.390pg4b & Blackwater Characters 1851. & 1851 & On Drawing & $\begin{array}{l}\text { Canaan Valley, VA } \\
\text { (WV) }\end{array}$ & On Drawing & Men at Leisure \\
\hline
\end{tabular}




\begin{tabular}{|c|c|c|c|c|c|c|c|}
\hline Number & Accession Number & Title & Date & $\begin{array}{l}\text { Source of Date } \\
\text { Information }\end{array}$ & Location & $\begin{array}{l}\text { Source of Location } \\
\text { Information }\end{array}$ & Themes \\
\hline 109 & W1995.030.393pg5a & $\begin{array}{l}\text { Blowing bladders - Hogs. A } \\
\text { Swallowing Match. }\end{array}$ & Unknown & $\begin{array}{l}\text { No Date on } \\
\text { Drawing }\end{array}$ & Unknown & No Location on Drawing & $\begin{array}{l}\text { African } \\
\text { Americans, } \\
\text { Animals } \\
\text { (Domestic), } \\
\text { Men at Work }\end{array}$ \\
\hline 110 & W1995.030.394pg5 & $\begin{array}{l}\text { Bluefish - length } 27 \text { inches - } \\
\text { weight } 51 / 4 \mathrm{lbs} / \mathrm{Scuppang}\end{array}$ & Unknown & $\begin{array}{l}\text { No Date on } \\
\text { Drawing }\end{array}$ & Unknown & No Location on Drawing & Animals (Wild) \\
\hline 111 & W1995.030.394pg4 & Bluefishing. Buzzards Bay & May 26,1859 & On Drawing & Buzzards Bay, MA & On Drawing & $\begin{array}{l}\text { Animals } \\
\text { (Wild), } \\
\text { Landscape } \\
\text { (Lakes), Men } \\
\text { at Work, } \\
\text { Transportation } \\
\text { (Boats) }\end{array}$ \\
\hline 112 & W1995.030.165a & Boring in the 270 foot gallery. & Unknown & $\begin{array}{l}\text { No Date on } \\
\text { Drawing }\end{array}$ & Unknown & No Location on Drawing & $\begin{array}{l}\text { African } \\
\text { Americans, } \\
\text { Children, } \\
\text { Industry, Men } \\
\text { at Work, } \\
\text { Women }\end{array}$ \\
\hline 113 & W1995.030.068 & Brashear City Depot & Unknown & $\begin{array}{l}\text { Date on Drawing } \\
\text { Unclear }\end{array}$ & Brashear City, LA & On Drawing & $\begin{array}{l}\text { African } \\
\text { Americans, } \\
\text { Architecture } \\
\text { (Towns), } \\
\text { Landscape } \\
\text { (Rivers), Men } \\
\text { at Work, } \\
\text { Transportation } \\
\text { (Boats) }\end{array}$ \\
\hline
\end{tabular}




\begin{tabular}{|c|c|c|c|c|c|c|c|}
\hline Number & Accession Number & Title & Date & $\begin{array}{l}\text { Source of Date } \\
\text { Information }\end{array}$ & Location & $\begin{array}{l}\text { Source of Location } \\
\text { Information }\end{array}$ & Themes \\
\hline 114 & W1995.030.313 & $\begin{array}{l}\text { Bride's Veil Weyer's Cave } \\
\text { Octo: 14.th..1853 }\end{array}$ & $\begin{array}{l}\text { October } 14, \\
1853\end{array}$ & On Drawing & Weyer's Cave, VA & On Drawing & $\begin{array}{l}\text { Landscape } \\
\text { (Caves), Men }\end{array}$ \\
\hline 115 & W1995.030.088 & Bridge of Sighs- Ah! Oh! & $\begin{array}{l}\text { September 2, } \\
\quad 1871\end{array}$ & $\begin{array}{c}\text { WVRHC } \\
\text { Description Page }\end{array}$ & Italy & & $\begin{array}{l}\text { Architecture } \\
\text { (Bridges), } \\
\text { Architecture } \\
\text { (Towns), } \\
\text { Landscape } \\
\text { (Rivers), } \\
\text { Transportation } \\
\text { (Boats) }\end{array}$ \\
\hline 116 & W1995.030.091 & Bridge of Trevi-Roman & May 6, 1887 & $\begin{array}{c}\text { WVRHC } \\
\text { Description Page }\end{array}$ & Italy & & $\begin{array}{l}\text { Landscape } \\
\text { (Mountains) }\end{array}$ \\
\hline 117 & W1995.030.052 & Butte la Rose & Unknown & $\begin{array}{l}\text { Date on Drawing } \\
\text { Unclear }\end{array}$ & Unknown & No Location on Drawing & $\begin{array}{l}\text { African } \\
\text { Americans, } \\
\text { Architecture, } \\
\text { Landscape } \\
\text { (Rivers), Men, } \\
\text { Military }\end{array}$ \\
\hline 118 & W1995.030.387pg2 & $\begin{array}{l}\text { Buzzards Old Shop Berkeley } \\
\text { Springs }\end{array}$ & Unknown & $\begin{array}{l}\text { No Date on } \\
\text { Drawing }\end{array}$ & $\begin{array}{l}\text { Berkeley Springs, VA } \\
\text { (WV) }\end{array}$ & On Drawing & $\begin{array}{l}\text { Architecture, } \\
\text { Landscape } \\
\text { (Fences), } \\
\text { Transportation } \\
\text { (Carriages) }\end{array}$ \\
\hline 119 & W1995.030.074 & Camp at Magruder's & $\begin{array}{l}\text { November 5, } \\
\quad 1861\end{array}$ & On Drawing & Unknown & No Location on Drawing & $\begin{array}{l}\text { Men in } \\
\text { Wilderness }\end{array}$ \\
\hline 120 & W1995.030.062 & Camp Barber Pleasant Valley & October 1862 & On Drawing & Unknown & No Location on Drawing & Men at Work \\
\hline
\end{tabular}




\begin{tabular}{|c|c|c|c|c|c|c|c|}
\hline Number & Accession Number & Title & Date & $\begin{array}{l}\text { Source of Date } \\
\text { Information }\end{array}$ & Location & $\begin{array}{l}\text { Source of Location } \\
\text { Information }\end{array}$ & Themes \\
\hline 121 & W1995.030.357 & $\begin{array}{l}\text { Camp of Topographical } \\
\text { Engineers Da(rr)ostown } \\
\text { Montgomery Co. Md.. Sept } \\
\text { 10th..1861 }\end{array}$ & $\begin{array}{l}\text { September } \\
10,1861\end{array}$ & On Drawing & $\begin{array}{c}\text { Montgomery County, } \\
\text { MD }\end{array}$ & On Drawing & Military \\
\hline 122 & W1995.030.387pg35b & Camp on the Lake & Unknown & $\begin{array}{l}\text { No Date on } \\
\text { Drawing }\end{array}$ & Unknown & No Location on Drawing & $\begin{array}{l}\text { Men in } \\
\text { Wilderness }\end{array}$ \\
\hline 123 & W1995.030.089 & Campanoli Bolognese & $\begin{array}{l}\text { April 22, } \\
1845\end{array}$ & On Drawing & Italy & & $\begin{array}{l}\text { Landscape } \\
\text { (Roads), } \\
\text { Women }\end{array}$ \\
\hline 124 & W1995.030.393pg37 & Cannel Coal. & 1867 & $\begin{array}{c}\text { WVRHC } \\
\text { Description Page }\end{array}$ & Unknown & No Location on Drawing & $\begin{array}{l}\text { Men at Work, } \\
\text { Transportation } \\
\text { (Boats) }\end{array}$ \\
\hline 125 & W1995.030.133 & $\begin{array}{l}\text { Capital Baton Rouge Feb. 2, } \\
1873 \text { from the (illegible) birth }\end{array}$ & $\begin{array}{l}\text { February 2, } \\
\quad 1873\end{array}$ & On Drawing & Baton Rouge, LA & On Drawing & $\begin{array}{l}\text { Architecture } \\
\text { (Institutional } \\
\text { Buildings) }\end{array}$ \\
\hline 126 & W1995.030.173 & $\begin{array}{l}\text { Carolina Buggies - Gates Co. - } \\
\text { at Belvedere April 8th } 1856 .\end{array}$ & April 8, 1856 & On Drawing & Belvedere, NC & On Drawing & $\begin{array}{l}\text { Transportation } \\
\text { (Carriages) }\end{array}$ \\
\hline 127 & W1995.030.031 & Cars & $\begin{array}{l}\text { March 16, } \\
1872\end{array}$ & On Drawing & Unknown & No Location on Drawing & $\begin{array}{l}\text { Transportation } \\
\text { (Carriages), } \\
\text { Women }\end{array}$ \\
\hline 128 & W1995.030.379 & $\begin{array}{l}\text { Cascades of the Blackwater } \\
\text { (Virginia) (Ca. 1852) }\end{array}$ & 1852 & $\begin{array}{c}\text { WVRHC } \\
\text { Description Page }\end{array}$ & $\begin{array}{l}\text { Canaan Valley, VA } \\
\text { (WV) }\end{array}$ & On Drawing & $\begin{array}{l}\text { Landscape } \\
\text { (Waterfalls) }\end{array}$ \\
\hline 129 & W1995.030.395pg14b & $\begin{array}{l}\text { Castle Rocks Kanawha May } \\
11 \text { th } 1872 .\end{array}$ & May 11,1872 & On Drawing & $\begin{array}{c}\text { Kanawha County, VA } \\
\text { (WV) }\end{array}$ & On Drawing & $\begin{array}{l}\text { Landscape } \\
\text { (Mountains), } \\
\text { Men in } \\
\text { Wilderness, } \\
\text { Women in } \\
\text { Wilderness }\end{array}$ \\
\hline
\end{tabular}




\begin{tabular}{|c|c|c|c|c|c|c|c|}
\hline Number & Accession Number & Title & Date & $\begin{array}{l}\text { Source of Date } \\
\text { Information }\end{array}$ & Location & $\begin{array}{l}\text { Source of Location } \\
\text { Information }\end{array}$ & Themes \\
\hline 130 & W1995.030.305 & $\begin{array}{l}\text { Cataract Weyer's Cave-Aug. } \\
\text { 14th..1853 }\end{array}$ & $\begin{array}{l}\text { August 14, } \\
1853\end{array}$ & On Drawing & Weyer's Cave, VA & On Drawing & $\begin{array}{l}\text { Landscape } \\
\text { (Caves), Men }\end{array}$ \\
\hline 131 & W1995.030.004 & Cathcing Hogs & $\begin{array}{l}\text { December 2, } \\
1857\end{array}$ & On Drawing & Unknown & No Location on Drawing & $\begin{array}{l}\text { African } \\
\text { Americans, } \\
\text { Animals } \\
\text { (Domestic), } \\
\text { Men at Work }\end{array}$ \\
\hline 132 & W1995.030.328 & $\begin{array}{l}\text { Cathedral Rock Pendleton Co } \\
\text { West Va Seneca } \\
\text { Oct..9th..1854 }\end{array}$ & $\begin{array}{l}\text { October } 9 \\
1854\end{array}$ & On Drawing & $\begin{array}{l}\text { Pendleton County, VA } \\
\text { (WV) }\end{array}$ & On Drawing & $\begin{array}{l}\text { Landscape } \\
\text { (Mountains) }\end{array}$ \\
\hline 133 & W1995.030.342 & $\begin{array}{l}\text { Cathedral Rock. Oct. } \\
\text { 9th..1854 Seneca. Pendleton } \\
\text { Co. West Va.. }\end{array}$ & $\begin{array}{l}\text { October } 9 \\
1854\end{array}$ & On Drawing & $\begin{array}{l}\text { Pendleton County, VA } \\
\text { (WV) }\end{array}$ & On Drawing & $\begin{array}{l}\text { Landscape } \\
\text { (Mountains) }\end{array}$ \\
\hline 134 & W1995.030.387pg31 & $\begin{array}{l}\text { Cathedral: Oct: 14th } 1853 \text { - } \\
\text { Wyer's Cave }\end{array}$ & $\begin{array}{l}\text { October } 14 \\
1853\end{array}$ & On Drawing & Weyer's Cave, VA & On Drawing & $\begin{array}{l}\text { Landscape } \\
\text { (Caves) }\end{array}$ \\
\hline 135 & W1995.030.303 & $\begin{array}{l}\text { Cedar Island Life Saving } \\
\text { Station Accomac Co. Va. Apr } \\
231887\end{array}$ & $\begin{array}{l}\text { April 23, } \\
1887\end{array}$ & On Drawing & Accomack County, VA & On Drawing & $\begin{array}{l}\text { Architecture, } \\
\text { Men at Work, } \\
\text { Transportation } \\
\text { (Boats) }\end{array}$ \\
\hline 136 & W1995.030.300 & $\begin{array}{l}\text { Central View Looking toward } \\
\text { Southern Entrance Natural } \\
\text { Tunnel, Scott Co. Virginia }\end{array}$ & $\begin{array}{l}\text { November } \\
23,1856\end{array}$ & On Drawing & Scott County, VA & On Drawing & $\begin{array}{l}\text { Landscape } \\
\text { (Caves), Men, } \\
\text { Women }\end{array}$ \\
\hline 137 & W1995.030.126 & $\begin{array}{l}\text { Century Plant New Orleans } \\
\text { March 25th } 1868\end{array}$ & $\begin{array}{l}\text { March 25, } \\
1868\end{array}$ & On Drawing & New Orleans, LA & On Drawing & Landscape \\
\hline 138 & W1995.030.082 & $\begin{array}{l}\text { Chamber (?) in Paradisino } \\
\text { Vallombrosa }\end{array}$ & Unknown & $\begin{array}{l}\text { No Date on } \\
\text { Drawing }\end{array}$ & Vallombrosa, Italy & On Drawing & $\begin{array}{l}\text { Copies of } \\
\text { European } \\
\text { Artwork }\end{array}$ \\
\hline
\end{tabular}




\begin{tabular}{|c|c|c|c|c|c|c|c|}
\hline Number & Accession Number & Title & Date & $\begin{array}{l}\text { Source of Date } \\
\text { Information }\end{array}$ & Location & $\begin{array}{l}\text { Source of Location } \\
\text { Information }\end{array}$ & Themes \\
\hline 139 & W1995.030.394pg25 & $\begin{array}{l}\text { Charleston Va. Oct. } 26 . \\
\text { Listening to the Indictment } \\
\text { and Pleadings, Copeland } 1859\end{array}$ & $\begin{array}{l}\text { October } 26 \\
1859\end{array}$ & On Drawing & $\begin{array}{l}\text { Charles Town, VA } \\
\text { (WV) }\end{array}$ & On Drawing & $\begin{array}{l}\text { Harper's Ferry } \\
\text { Raid Trials, } \\
\text { Men }\end{array}$ \\
\hline 140 & W1995.030.392pg7a & $\begin{array}{l}\text { Charleston W.Va Aug 31st } \\
1887\end{array}$ & $\begin{array}{l}\text { August 31, } \\
1887\end{array}$ & On Drawing & $\begin{array}{c}\text { Charles Town, VA } \\
\text { (WV) }\end{array}$ & On Drawing & $\begin{array}{l}\text { African } \\
\text { Americans, } \\
\text { Men at Leisure }\end{array}$ \\
\hline 141 & W1995.030.374 & $\begin{array}{l}\text { Charleston, Va. The Execution } \\
\text { of John Brown, December 2nd } \\
1859\end{array}$ & $\begin{array}{l}\text { December } 2 \\
\quad 1859\end{array}$ & On Drawing & $\begin{array}{c}\text { Charles Town, VA } \\
\text { (WV) }\end{array}$ & On Drawing & $\begin{array}{l}\text { Animals } \\
\text { (Domestic), } \\
\text { Architecture } \\
\text { (Residential } \\
\text { Buildings), } \\
\text { Harper's Ferry } \\
\text { Raid Trials, } \\
\text { Men, Military }\end{array}$ \\
\hline 142 & W1995.030.280 & Charlestown Aug 30th..1887 & $\begin{array}{l}\text { August } 30, \\
1887\end{array}$ & On Drawing & $\begin{array}{l}\text { Charles Town, VA } \\
\text { (WV) }\end{array}$ & On Drawing & Men \\
\hline 143 & W1995.030.365 & $\begin{array}{l}\text { Charlestown, Va.. Oct: 26th. } \\
1859\end{array}$ & $\begin{array}{l}\text { October } 26 \\
1859\end{array}$ & On Drawing & $\begin{array}{l}\text { Charles Town, VA } \\
\text { (WV) }\end{array}$ & On Drawing & Men \\
\hline 144 & W1995.030.271 & $\begin{array}{l}\text { Charley Bowers Jany } \\
21 \text { st..1876 }\end{array}$ & $\begin{array}{l}\text { January } 21 \\
1876\end{array}$ & On Drawing & Unknown & No Location on Drawing & Children \\
\hline 145 & W1995.030.393pg6a & Charly & Unknown & $\begin{array}{l}\text { No Date on } \\
\text { Drawing }\end{array}$ & Unknown & No Location on Drawing & $\begin{array}{l}\text { Animals } \\
\text { (Domestic), } \\
\text { Children, Men } \\
\text { at Work, Men } \\
\text { at Leisure, } \\
\text { Transportation } \\
\text { (Boats) }\end{array}$ \\
\hline
\end{tabular}




\begin{tabular}{|c|c|c|c|c|c|c|c|}
\hline Number & Accession Number & Title & Date & $\begin{array}{l}\text { Source of Date } \\
\text { Information }\end{array}$ & Location & $\begin{array}{l}\text { Source of Location } \\
\text { Information }\end{array}$ & Themes \\
\hline 146 & W1995.030.336 & $\begin{array}{l}\text { Chimney Rocks Hardy Co } \\
\text { West Va.. }\end{array}$ & Unknown & $\begin{array}{l}\text { No Date on } \\
\text { Drawing }\end{array}$ & $\begin{array}{l}\text { Hardy or Hampshire } \\
\text { County, VA (WV) }\end{array}$ & On Drawing & 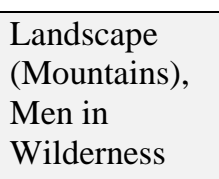 \\
\hline 147 & W1995.030.302 & $\begin{array}{l}\text { Chimneys Augusta Co. } \\
\text { Virginia }\end{array}$ & Unknown & $\begin{array}{l}\text { No Date on } \\
\text { Drawing }\end{array}$ & Augusta County, VA & On Drawing & $\begin{array}{l}\text { Landscape } \\
\text { (Mountains) }\end{array}$ \\
\hline 148 & W1995.030.253 & $\begin{array}{l}\text { Christmas 1867. Berkeley } \\
\text { Springs West Va. }\end{array}$ & $\begin{array}{c}\text { December } 25, \\
1867\end{array}$ & On Drawing & $\begin{array}{l}\text { Berkeley Springs, VA } \\
\text { (WV) }\end{array}$ & On Drawing & $\begin{array}{l}\text { Children, } \\
\text { Decorative } \\
\text { Arts }\end{array}$ \\
\hline 149 & W1995.030.151a & $\begin{array}{l}\text { City of Mexico 1880. Mrs. } \\
\text { Gadsden's Waiter }\end{array}$ & 1880 & On Drawing & Mexico City, Mexico & On Drawing & Men at Work \\
\hline 150 & W1995.030.290 & Clarke Co. Va. & $\begin{array}{l}\text { March 18, } \\
1859\end{array}$ & On Drawing & Clarke County, VA & On Drawing & Animals \\
\hline 151 & W1995.030.292 & Clarke Co. Va. & $\begin{array}{l}\text { March 14, } \\
1859\end{array}$ & On Drawing & Clarke County, VA & On Drawing & Animals \\
\hline 152 & W1995.030.349 & $\begin{array}{l}\text { Cliffs at Seneca Pendleton Co } \\
\text { West VaE }\end{array}$ & $\begin{array}{c}\text { December } 2 \\
1859\end{array}$ & $\begin{array}{c}\text { WVRHC } \\
\text { Description Page }\end{array}$ & $\begin{array}{l}\text { Pendleton County, VA } \\
\text { (WV) }\end{array}$ & On Drawing & $\begin{array}{l}\text { Architecture } \\
\text { (Residential } \\
\text { Buildings), } \\
\text { Landscape } \\
\text { (Mountains) }\end{array}$ \\
\hline 153 & W1995.030.265 & Col. Baker Bakersville N.C. & $\begin{array}{l}\text { December } 5 \\
1856\end{array}$ & On Drawing & Bakersville, NC & On Drawing & Men \\
\hline 154 & W1995.030.390pg23 & Col. Sancendaffer's coat & May 7, 1859 & $\begin{array}{c}\text { WVRHC } \\
\text { Description Page }\end{array}$ & Unknown & No Location on Drawing & Men, Military \\
\hline 155 & W1995.030.024 & $\begin{array}{l}\text { Cole and Mayer's Excursion } \\
\text { Balt \& Ohio R.R. }\end{array}$ & June 16,1860 & On Drawing & Unknown & No Location on Drawing & Animals \\
\hline 156 & W1995.030.393pg35a & Comfort. Kanawha - 1871. & 1871 & On Drawing & $\begin{array}{c}\text { Kanawha County, VA } \\
\text { (WV) }\end{array}$ & On Drawing & Men at Leisure \\
\hline
\end{tabular}




\begin{tabular}{|c|c|c|c|c|c|c|c|}
\hline Number & Accession Number & Title & Date & $\begin{array}{l}\text { Source of Date } \\
\text { Information }\end{array}$ & Location & $\begin{array}{l}\text { Source of Location } \\
\text { Information }\end{array}$ & Themes \\
\hline 157 & W1995.030.035 & Connelsville Railway & $\begin{array}{c}\text { October } 4, \\
1871\end{array}$ & On Drawing & Unknown & No Location on Drawing & Men \\
\hline 158 & W1995.030.054 & Conscientious Trooper & October 1848 & $\begin{array}{c}\text { WVRHC } \\
\text { Description Page }\end{array}$ & Unknown & No Location on Drawing & $\begin{array}{l}\text { Animals } \\
\text { (Domestic), } \\
\text { Men, Military }\end{array}$ \\
\hline 159 & W1995.030.098 & Convent Gate Vallombrosa & $\begin{array}{l}\text { September } 9 \text {, } \\
1876\end{array}$ & $\begin{array}{c}\text { WVRHC } \\
\text { Description Page }\end{array}$ & Vallombrosa, Italy & On Drawing & $\begin{array}{l}\text { Religious } \\
\text { Shrines }\end{array}$ \\
\hline 160 & W1995.030.390pg18a & Conway 1852 Blackwater & 1852 & On Drawing & $\begin{array}{c}\text { Canaan Valley, VA } \\
\text { (WV) }\end{array}$ & On Drawing & $\begin{array}{l}\text { Architecture } \\
\text { (Towns), Men } \\
\text { at Work }\end{array}$ \\
\hline 161 & W1995.030.394pg26 & $\begin{array}{l}\text { Copeland Green Listening to } \\
\text { the Indictment. Charleston, } \\
\text { Virginia, Oct. } 26,1859\end{array}$ & $\begin{array}{l}\text { October 26, } \\
1859\end{array}$ & On Drawing & $\begin{array}{c}\text { Charles Town, VA } \\
\text { (WV) }\end{array}$ & On Drawing & $\begin{array}{l}\text { African } \\
\text { Americans, } \\
\text { Harper's Ferry } \\
\text { Raid Trials, } \\
\text { Men }\end{array}$ \\
\hline 162 & W1995.030.037 & Copies from Pompei & April 1875 & On Drawing & Unknown & No Location on Drawing & $\begin{array}{l}\text { Copies of } \\
\text { European } \\
\text { Artwork }\end{array}$ \\
\hline 163 & W1995.030.040 & Copies from the Japanese & April 1875 & On Drawing & Unknown & No Location on Drawing & $\begin{array}{l}\text { Copies of } \\
\text { Japanese } \\
\text { Artwork }\end{array}$ \\
\hline 164 & W1995.030.041 & Corner in the Pork & $\begin{array}{l}\text { April 25, } \\
1887\end{array}$ & On Drawing & Unknown & No Location on Drawing & $\begin{array}{l}\text { Animals } \\
\text { (Domestic) }\end{array}$ \\
\hline 165 & W1995.030.394pg14a & $\begin{array}{l}\text { Crawford Notch White } \\
\text { Mountains }\end{array}$ & June 25,1859 & On Drawing & White Mountains, NH & On Drawing & Animals (Wild) \\
\hline
\end{tabular}




\begin{tabular}{|c|c|c|c|c|c|c|c|}
\hline Number & Accession Number & Title & Date & $\begin{array}{l}\text { Source of Date } \\
\text { Information }\end{array}$ & Location & $\begin{array}{l}\text { Source of Location } \\
\text { Information }\end{array}$ & Themes \\
\hline 166 & W1995.030.320 & $\begin{array}{l}\text { Crown of Otter Botetourt Co. } \\
\text { Virginia Sketch commenced- } \\
\text { Nov; } 1853 \text {-completed Sept: } \\
\text { 21st..1872 }\end{array}$ & $\begin{array}{c}\text { November } \\
1853 / \text { Septem } \\
\text { ber } 21,1872\end{array}$ & On Drawing & Botetourt County, VA & On Drawing & $\begin{array}{l}\text { Landscape } \\
\text { (Mountains) }\end{array}$ \\
\hline 167 & W1995.030.394pg12 & $\begin{array}{l}\text { Crystal Cascade White } \\
\text { (mount) }\end{array}$ & June 25,1854 & On Drawing & White Mountains, NH & On Drawing & $\begin{array}{l}\text { Landscape } \\
\text { (Mountains), } \\
\text { Landscape } \\
\text { (Waterfalls) }\end{array}$ \\
\hline 168 & W1995.030.030 & $\begin{array}{l}\text { Cupid on a Bust / B. \& O. } \\
\text { R.R. Train }\end{array}$ & $\begin{array}{l}\text { March 16, } \\
\quad 1872\end{array}$ & On Drawing & Unknown & No Location on Drawing & $\begin{array}{l}\text { Decorative } \\
\text { Arts, Men }\end{array}$ \\
\hline 169 & W1995.030.392pg8a & $\begin{array}{l}\text { Custis Arms. The Tomb of } \\
\text { Arlington. May 6th } 1887\end{array}$ & May 6, 1887 & On Drawing & Arlington, VA & On Drawing & Cemeteries \\
\hline 170 & W1995.030.387pg34b & Dam No 6 Potomac River Va & Unknown & $\begin{array}{l}\text { No Date on } \\
\text { Drawing }\end{array}$ & Potomac River, VA & On Drawing & $\begin{array}{l}\text { Landscape } \\
\text { (Rivers) }\end{array}$ \\
\hline 171 & W1995.030.387pg23b & David F. Foster & $\begin{array}{l}\text { December } 9 \\
1858\end{array}$ & On Drawing & Unknown & No Location on Drawing & Children \\
\hline 172 & W1995.030.394pg17 & $\begin{array}{l}\text { Deacon Simons Toad Rock } \\
\text { Vinyard/(Drawing of a Baby) }\end{array}$ & June 1859 & On Drawing & Unknown & No Location on Drawing & $\begin{array}{l}\text { Children, } \\
\text { Landscape }\end{array}$ \\
\hline 173 & $\begin{array}{l}\text { W1995.030.393pg36b } \\
\mathrm{c}\end{array}$ & $\begin{array}{l}\text { Dec 3rd 1856. View of the } \\
\text { Roane Mountn from the } \\
\text { Tennesee line - Summit of } \\
\text { Iron Mountns }\end{array}$ & $\begin{array}{l}\text { December 3, } \\
1856\end{array}$ & On Drawing & Iron Mountains, TN & On Drawing & $\begin{array}{l}\text { Architecture } \\
\text { (Residential } \\
\text { Buildings), } \\
\text { Landscape } \\
\text { (Mountains), } \\
\text { Men in } \\
\text { Wilderness }\end{array}$ \\
\hline 174 & W1995.030.387pg26b & Dec. 4th 1857 Clarke Co Va & $\begin{array}{c}\text { December } 4 \\
1857\end{array}$ & On Drawing & Clarke County, VA & On Drawing & $\begin{array}{l}\text { Architecture } \\
\text { (Residential } \\
\text { Buildings), } \\
\text { Landscape } \\
\text { (Fences) }\end{array}$ \\
\hline
\end{tabular}




\begin{tabular}{|c|c|c|c|c|c|c|c|}
\hline Number & Accession Number & Title & Date & $\begin{array}{l}\text { Source of Date } \\
\text { Information }\end{array}$ & Location & $\begin{array}{l}\text { Source of Location } \\
\text { Information }\end{array}$ & Themes \\
\hline 175 & W1995.030.249a & $\begin{array}{l}\text { December 15th } 186711 \text { 1/2 } \\
\text { months old }\end{array}$ & $\begin{array}{c}\text { December } 15, \\
1857\end{array}$ & On Drawing & Unknown & No Location on Drawing & Children \\
\hline 176 & W1995.030.393pg33b & $\begin{array}{l}\text { Deck Passenger. Ginger Lakes } \\
\text { [illegible word] }\end{array}$ & Unknown & $\begin{array}{l}\text { No Date on } \\
\text { Drawing }\end{array}$ & Finger Lakes, NY & On Drawing & $\begin{array}{l}\text { Men at Work, } \\
\text { Women at } \\
\text { Work }\end{array}$ \\
\hline 177 & W1995.030.393pg20a & Deck Passenger. Kanawha & Unknown & $\begin{array}{l}\text { No Date on } \\
\text { Drawing }\end{array}$ & $\begin{array}{c}\text { Kanawha County, VA } \\
\text { (WV) }\end{array}$ & On Drawing & Men, Woman \\
\hline 178 & W1995.030.324 & $\begin{array}{l}\text { Delights of Berkeley. } \\
\text { Returning from the Evening } \\
\text { Bath escorted by a flash of } \\
\text { Lightning Aug } 12.1872\end{array}$ & $\begin{array}{l}\text { August 12, } \\
1872\end{array}$ & On Drawing & $\begin{array}{l}\text { Berkeley Springs, VA } \\
\text { (WV) }\end{array}$ & On Drawing & Men, Women \\
\hline 179 & W1995.030.387pg20 & $\begin{array}{l}\text { Delmonico's Dec 16th } 1858 \\
\text { Berkeley Springs October } \\
1858\end{array}$ & $\begin{array}{c}\text { April 19, } \\
1859\end{array}$ & On Drawing & $\begin{array}{l}\text { Berkeley Springs, VA } \\
\text { (WV) }\end{array}$ & On Drawing & $\begin{array}{l}\text { Animals } \\
\text { (Domestic), } \\
\text { Men at Leisure }\end{array}$ \\
\hline 180 & W1995.030.164 & $\begin{array}{l}\text { Descending the pump shaft } \\
\text { April 25th 1856 8:o clock }\end{array}$ & $\begin{array}{l}\text { April 25, } \\
1856\end{array}$ & On Drawing & Unknown & No Location on Drawing & $\begin{array}{l}\text { Industry, Men } \\
\text { at Work }\end{array}$ \\
\hline 181 & W1995.030.391pg8a & $\begin{array}{l}\text { Descending the Shaft at Ore } \\
\text { Knob July } 1874 .\end{array}$ & July 1874 & On Drawing & Guilford, NC & See Number 266 & $\begin{array}{l}\text { Industry, Men } \\
\text { at Work }\end{array}$ \\
\hline 182 & W1995.030.393pg2a & $\begin{array}{l}\text { Distillery - Apple Jack - } \\
\text { Yancey Co. N.C. }\end{array}$ & Unknown & $\begin{array}{l}\text { No Date on } \\
\text { Drawing }\end{array}$ & Yancey County, NC & On Drawing & $\begin{array}{l}\text { Architecture, } \\
\text { Industry }\end{array}$ \\
\hline 183 & W1995.030.393pg4b & Dogs and man and woman & Unknown & $\begin{array}{l}\text { No Date on } \\
\text { Drawing }\end{array}$ & Unknown & No Location on Drawing & $\begin{array}{l}\text { Animals } \\
\text { (Domestic), } \\
\text { Children, Men } \\
\text { at Leisure }\end{array}$ \\
\hline
\end{tabular}




\begin{tabular}{|c|c|c|c|c|c|c|c|}
\hline Number & Accession Number & Title & Date & $\begin{array}{l}\text { Source of Date } \\
\text { Information }\end{array}$ & Location & $\begin{array}{l}\text { Source of Location } \\
\text { Information }\end{array}$ & Themes \\
\hline 184 & W1995.030.387pg50 & $\begin{array}{l}\text { Dove Baggage Master Dec. } \\
\text { 1st 1858. Baltimore }\end{array}$ & $\begin{array}{l}\text { December 1, } \\
1858\end{array}$ & On Drawing & Baltimore, MD & On Drawing & Men at Work \\
\hline 185 & W1995.030.140 & $\begin{array}{l}\text { Draw Bridge on the Shell } \\
\text { Road From New Orleans to } \\
\text { Lake Ponchartrain }\end{array}$ & $\begin{array}{l}\text { October } 18 \\
1853\end{array}$ & $\begin{array}{c}\text { WVRHC } \\
\text { Description Page }\end{array}$ & New Orleans, LA & On Drawing & $\begin{array}{l}\text { Architecture } \\
\text { (Bridges), } \\
\text { Architecture } \\
\text { (Towns), } \\
\text { Landscape } \\
\text { (Rivers) }\end{array}$ \\
\hline 186 & W1995.030.346 & $\begin{array}{l}\text { Drawn April 1st..1859. Locust } \\
\text { Grove. } 20 \text { years ago }\end{array}$ & April 1, 1859 & On Drawing & $\begin{array}{l}\text { Charles Town, VA } \\
\text { (WV) }\end{array}$ & $\begin{array}{c}\text { Cecil Eby, "Porte } \\
\text { Crayon": The Life of } \\
\text { David Hunter Strother, } \\
\text { (Chapel Hill, NC: } \\
\text { University of North } \\
\text { Carolina Press, 1960), } 7 .\end{array}$ & $\begin{array}{l}\text { African } \\
\text { Americans, } \\
\text { Animals } \\
\text { (Domestic), } \\
\text { Architecture } \\
\text { (Residential } \\
\text { Buildings), } \\
\text { Children, Men, } \\
\text { Women }\end{array}$ \\
\hline 187 & W1995.030.296 & $\begin{array}{l}\text { Drummond The Lake of the } \\
\text { Dismal Swamp }\end{array}$ & June 25,1859 & $\begin{array}{c}\text { WVRHC } \\
\text { Description Page }\end{array}$ & Dismal Swamp, VA & On Drawing & $\begin{array}{l}\text { Landscape } \\
\text { (Lakes) }\end{array}$ \\
\hline 188 & W1995.030.018 & Edgartown & $\begin{array}{l}\text { May 7, June } \\
5 \text {, and June 6, } \\
1859\end{array}$ & On Drawing & $\begin{array}{c}\text { Edgarton, NY/ } \\
\text { Nantucket, MA/ MD }\end{array}$ & On Drawing & Men \\
\hline 189 & W1995.030.390pg15a & Edmund Thornhill & Unknown & $\begin{array}{l}\text { No Date on } \\
\text { Drawing }\end{array}$ & Unknown & No Location on Drawing & Men at Leisure \\
\hline 190 & W1995.030.070 & $\begin{array}{l}\text { Edward's Ferry The Day After } \\
\text { Battle at Balls Bluff }\end{array}$ & Unknown & $\begin{array}{l}\text { No Date on } \\
\text { Drawing }\end{array}$ & Loudon County, VA & $\frac{\text { http://www.nps.gov/abpp/ }}{\underline{\text { battles/va006.htm }}}$ & 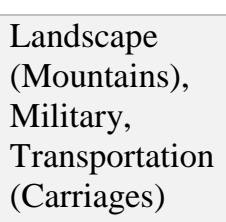 \\
\hline
\end{tabular}




\begin{tabular}{|c|c|c|c|c|c|c|c|}
\hline Number & Accession Number & Title & Date & $\begin{array}{l}\text { Source of Date } \\
\text { Information }\end{array}$ & Location & $\begin{array}{l}\text { Source of Location } \\
\text { Information }\end{array}$ & Themes \\
\hline 191 & W1995.030.394pg28 & $\begin{array}{l}\text { Edwin Coppick (sic) on Trial. } \\
\text { Aged 24, Oct. 26, (1859) } \\
\text { Charleston, Va }\end{array}$ & $\begin{array}{l}\text { October 26, } \\
1859\end{array}$ & $\begin{array}{c}\text { WVRHC } \\
\text { Description Page }\end{array}$ & $\begin{array}{l}\text { Charles Town, VA } \\
\text { (WV) }\end{array}$ & On Drawing & $\begin{array}{l}\text { Harper's Ferry } \\
\text { Raid Trials, } \\
\text { Men }\end{array}$ \\
\hline 192 & W1995.030.267a & $\begin{array}{l}\text { Egg Boy Berkeley Springs } \\
\text { December 18th, } 1873\end{array}$ & $\begin{array}{c}\text { December } 18, \\
1873\end{array}$ & On Drawing & $\begin{array}{c}\text { Berkeley Springs, VA } \\
\text { (WV) }\end{array}$ & On Drawing & Men at Work \\
\hline 193 & W1995.030.387pg37 & $\begin{array}{l}\text { Emily [Strother] and Mahala } \\
\text { July 8th } 1857\end{array}$ & July 8,1857 & On Drawing & Unknown & No Location on Drawing & Women \\
\hline 194 & W1995.030.384 & $\begin{array}{l}\text { Encampment No 3. on the } \\
\text { Canaan Fork }\end{array}$ & Unknown & $\begin{array}{l}\text { No Date on } \\
\text { Drawing }\end{array}$ & $\begin{array}{c}\text { Canaan Valley, VA } \\
\text { (WV) }\end{array}$ & On Drawing & $\begin{array}{l}\text { Architecture, } \\
\text { Men in } \\
\text { Wilderness }\end{array}$ \\
\hline 195 & W1995.030.386pg1b & $\begin{array}{l}\text { Encampment No. 2. The } \\
\text { Mummy }\end{array}$ & 1852 & On Drawing & $\begin{array}{c}\text { Canaan Valley, VA } \\
\text { (WV) }\end{array}$ & $\begin{array}{l}\text { Strother, "The Virginian } \\
\text { Canaan," Harpers New } \\
\text { Monthly Magazine, } \\
\text { December } 1853,27 .\end{array}$ & $\begin{array}{l}\text { Men in } \\
\text { Wilderness }\end{array}$ \\
\hline 196 & W1995.030.356 & $\begin{array}{l}\text { Encampment of Alabama \& } \\
\text { Mississippi Volunteers } \\
\text { Harpers ferry Va.. May } \\
\text { 27th..1861 }\end{array}$ & May 27, 1861 & On Drawing & $\begin{array}{l}\text { Harpers Ferry, VA } \\
\text { (WV) }\end{array}$ & On Drawing & $\begin{array}{l}\text { Animals } \\
\text { (Domestic), } \\
\text { Children, } \\
\text { Landscape } \\
\text { (Mountains), } \\
\text { Men, Military }\end{array}$ \\
\hline 197 & W1995.030.067 & $\begin{array}{l}\text { Encampment of } \\
\text { Topographical Engineers at } \\
\text { the foot of Sugarloaf } \\
\text { Mountain Frederick Co. Md }\end{array}$ & 1861 & On Drawing & Frederick County, MD & On Drawing & $\begin{array}{l}\text { Architecture } \\
\text { (Residential } \\
\text { Buildings), } \\
\text { Animals } \\
\text { (Domestic), } \\
\text { Landscape } \\
\text { (Mountains), } \\
\text { Military, } \\
\text { Transportation } \\
\text { (Carriages) }\end{array}$ \\
\hline
\end{tabular}




\begin{tabular}{|c|c|c|c|c|c|c|c|}
\hline Number & Accession Number & Title & Date & $\begin{array}{l}\text { Source of Date } \\
\text { Information }\end{array}$ & Location & $\begin{array}{l}\text { Source of Location } \\
\text { Information }\end{array}$ & Themes \\
\hline 198 & W1995.030.358 & $\begin{array}{l}\text { Encampment of } \\
\text { Topographical Engineers near } \\
\text { Hyattstown, Montgomery Co. } \\
\text { Md. August 26th } 1861\end{array}$ & $\begin{array}{l}\text { August 26, } \\
1861\end{array}$ & On Drawing & $\begin{array}{c}\text { Montgomery County, } \\
\text { MD }\end{array}$ & On Drawing & Military \\
\hline 199 & W1995.030.380 & $\begin{array}{l}\text { Encampment on Skillet Fork } \\
\text { (Virginia Canaan 1852) }\end{array}$ & 1852 & On Drawing & $\begin{array}{c}\text { Canaan Valley, VA } \\
\text { (WV) }\end{array}$ & On Drawing & $\begin{array}{l}\text { Men in } \\
\text { Wilderness }\end{array}$ \\
\hline 200 & W1995.030.053 & $\begin{array}{l}\text { Encampment, entrance of the } \\
\text { Portsmouth guards,Jamestown } \\
\text { Island Va. }\end{array}$ & May 23, 1861 & $\begin{array}{c}\text { WVRHC } \\
\text { Description Page }\end{array}$ & Jamestown, VA & On Drawing & $\begin{array}{l}\text { Animals } \\
\text { (Domestic), } \\
\text { Men, Military, } \\
\text { Transportation } \\
\text { (Boats) }\end{array}$ \\
\hline 201 & W1995.030.285 & $\begin{array}{l}\text { Entrance to Nick a Jack Cave } \\
\text { Near Chattanooga Tennessee }\end{array}$ & $\begin{array}{l}\text { October } 26 \\
1859\end{array}$ & $\begin{array}{c}\text { WVRHC } \\
\text { Description Page }\end{array}$ & Chattanooga, TN & On Drawing & $\begin{array}{l}\text { Landscape } \\
\text { (Mountains) }\end{array}$ \\
\hline 202 & W1995.030.338 & $\begin{array}{l}\text { Entrance to Weyer's Cave } \\
\text { Saturday October 14th.. } 1853\end{array}$ & $\begin{array}{l}\text { October 14, } \\
1853\end{array}$ & On Drawing & Weyer's Cave, VA & On Drawing & $\begin{array}{l}\text { Landscape } \\
\text { (Mountains), } \\
\text { Men in } \\
\text { Wilderness, } \\
\text { Women in } \\
\text { Wilderness }\end{array}$ \\
\hline 203 & W1995.030.093 & Environs of Rome & March 1842 & On Drawing & Rome, Italy & On Drawing & $\begin{array}{l}\text { Architecture } \\
\text { (Towns), } \\
\text { Landscape }\end{array}$ \\
\hline 204 & W1995.030.386pg3 & Falls of Canaan Fork & Unknown & Unknown & $\begin{array}{l}\text { Canaan Valley, VA } \\
\text { (WV) }\end{array}$ & $\begin{array}{l}\text { WVRHC Description } \\
\text { Page }\end{array}$ & $\begin{array}{l}\text { Landscape } \\
\text { (Waterfalls) }\end{array}$ \\
\hline
\end{tabular}




\begin{tabular}{|c|c|c|c|c|c|c|c|}
\hline Number & Accession Number & Title & Date & $\begin{array}{l}\text { Source of Date } \\
\text { Information }\end{array}$ & Location & $\begin{array}{l}\text { Source of Location } \\
\text { Information }\end{array}$ & Themes \\
\hline 205 & W1995.030.386 & Falls of the Canaan Fork & Unknown & Unknown & $\begin{array}{c}\text { Canaan Valley, VA } \\
\text { (WV) }\end{array}$ & $\begin{array}{c}\text { WVRHC Description } \\
\text { Page }\end{array}$ & $\begin{array}{l}\text { Landscape } \\
\text { (Waterfalls) }\end{array}$ \\
\hline 206 & W1995.030.335 & $\begin{array}{l}\text { Fancellers Tub Mill-Mouth of } \\
\text { Blackwater }\end{array}$ & Unknown & $\begin{array}{l}\text { No Date on } \\
\text { Drawing }\end{array}$ & $\begin{array}{l}\text { Canaan Valley, VA } \\
\text { (WV) }\end{array}$ & On Drawing & $\begin{array}{l}\text { Animals, } \\
\text { Architecture, } \\
\text { Industry, } \\
\text { Landscape } \\
\text { (Rivers), Men } \\
\text { at Work }\end{array}$ \\
\hline 207 & W1995.030.327 & Fancy Ball at Berkeley & $\begin{array}{l}\text { February } 2 \\
\quad 1873\end{array}$ & $\begin{array}{c}\text { WVRHC } \\
\text { Description Page }\end{array}$ & $\begin{array}{l}\text { Berkeley Springs, VA } \\
\text { (WV) }\end{array}$ & On Drawing & Men at Work \\
\hline 208 & W1995.030.386pg4 & Fate of the Fancy Rods. & Unknown & $\begin{array}{l}\text { No Date on } \\
\text { Drawing }\end{array}$ & $\begin{array}{l}\text { Canaan Valley, VA } \\
\text { (WV) }\end{array}$ & $\begin{array}{c}\text { Strother, "The Virginian } \\
\text { Canaan," Harpers New } \\
\text { Monthly Magazine, } \\
\text { December } 1853,30 .\end{array}$ & $\begin{array}{l}\text { Men in } \\
\text { Wilderness }\end{array}$ \\
\hline 209 & W1995.030.198 & Feather in his Hat & 1845 & $\begin{array}{c}\text { WVRHC } \\
\text { Description Page }\end{array}$ & Unknown & No Location on Drawing & $\begin{array}{l}\text { Children, Men, } \\
\text { Military, } \\
\text { Women }\end{array}$ \\
\hline 210 & W1995.030.382b & First trout of $X$. & Unknown & $\begin{array}{l}\text { No Date on } \\
\text { Drawing }\end{array}$ & $\begin{array}{l}\text { Canaan Valley, VA } \\
\text { (WV) }\end{array}$ & $\begin{array}{l}\text { Strother, "The Virginian } \\
\text { Canaan," Harpers New } \\
\text { Monthly Magazine, } \\
\text { December 1853, } 33 .\end{array}$ & $\begin{array}{l}\text { Men in } \\
\text { Wilderness }\end{array}$ \\
\hline 211 & W1995.030.162 & Fishery Games May 5, 1859 & May 5,1859 & On Drawing & Unknown & No Location on Drawing & Men at Leisure \\
\hline 212 & W1995.030.083 & Fontana dei Capri, Tivoli & 1842 & On Drawing & Tivoli, Italy & On Drawing & $\begin{array}{l}\text { Animals } \\
\text { (Domestic), } \\
\text { Architecture, } \\
\text { Landscape } \\
\text { (Mountains), } \\
\text { Men }\end{array}$ \\
\hline 213 & W1995.030.086 & $\begin{array}{l}\text { Fontana della Piazza Barberini } \\
\text { Rome }\end{array}$ & 1842 & On Drawing & Rome, Italy & On Drawing & $\begin{array}{l}\text { Decorative } \\
\text { Arts }\end{array}$ \\
\hline
\end{tabular}




\begin{tabular}{|c|c|c|c|c|c|c|c|}
\hline Number & Accession Number & Title & Date & $\begin{array}{l}\text { Source of Date } \\
\text { Information }\end{array}$ & Location & $\begin{array}{l}\text { Source of Location } \\
\text { Information }\end{array}$ & Themes \\
\hline 214 & W1995.030.388pg23 & $\begin{array}{l}\text { Fool Van Vanmetre. Berkeley } \\
\text { Co; } 1845 .\end{array}$ & 1845 & On Drawing & $\begin{array}{l}\text { Berkeley Springs, VA } \\
\text { (WV) }\end{array}$ & On Drawing & Men \\
\hline 215 & W1995.030.147 & $\begin{array}{l}\text { Fort Fredrick from the High } \\
\text { Bridge over the Ches. and } \\
\text { Ohio Canal }\end{array}$ & $\begin{array}{l}\text { October 14, } \\
1853\end{array}$ & $\begin{array}{c}\text { WVRHC } \\
\text { Description Page }\end{array}$ & Frederick, MD & On Drawing & \\
\hline 216 & W1995.030.143a & $\begin{array}{l}\text { Four Oaks at the Villiers } \\
\text { Plantation below New Orleans } \\
\text { - The Brittish Surgical } \\
\text { Headquarters during the } \\
\text { Battle, and where General } \\
\text { Buckingham died Jan } 8,1815\end{array}$ & Unknown & $\begin{array}{l}\text { No Date on } \\
\text { Drawing }\end{array}$ & New Orleans, LA & On Drawing & $\begin{array}{l}\text { Landscape, } \\
\text { Military }\end{array}$ \\
\hline 217 & W1995.030.026 & Frank Brownings & May 25,1863 & On Drawing & Unknown & No Location on Drawing & $\begin{array}{l}\text { Architecture } \\
\text { (Residential } \\
\text { Buildings), } \\
\text { Men, Women }\end{array}$ \\
\hline 218 & W1995.030.390pg20a & Frank Peters at Towers & 1852 & On Drawing & $\begin{array}{l}\text { Canaan Valley, VA } \\
\text { (WV) }\end{array}$ & $\begin{array}{l}\text { Strother, "The Virginian } \\
\text { Canaan," Harpers New } \\
\text { Monthly Magazine, } \\
\text { December 1853, } 20 .\end{array}$ & Men at Leisure \\
\hline 219 & W1995.030.254 & $\begin{array}{l}\text { From a Daguerrotype. Dec. } \\
\text { 24th.. } 1860 .\end{array}$ & $\begin{array}{c}\text { December } 24, \\
1860\end{array}$ & On Drawing & Unknown & No Location on Drawing & $\begin{array}{l}\text { Decorative } \\
\text { Arts, Women }\end{array}$ \\
\hline 220 & W1995.030.069 & $\begin{array}{l}\text { From Genl. Stone's } \\
\text { Headquarters- Poolsville Md. }\end{array}$ & 1861 & On Drawing & Poolesville, MD & On Drawing & $\begin{array}{l}\text { Animals } \\
\text { (domestic), } \\
\text { Men, Military, } \\
\text { Transportation } \\
\text { (Carriages) }\end{array}$ \\
\hline
\end{tabular}




\begin{tabular}{|c|c|c|c|c|c|c|c|}
\hline Number & Accession Number & Title & Date & $\begin{array}{l}\text { Source of Date } \\
\text { Information }\end{array}$ & Location & $\begin{array}{l}\text { Source of Location } \\
\text { Information }\end{array}$ & Themes \\
\hline 221 & W1995.030.394pg2 & $\begin{array}{l}\text { From Mt. Washington looking } \\
\text { towards Crawfords }\end{array}$ & June 25,1859 & On Drawing & Mt. Washington, NH & On Drawing & $\begin{array}{l}\text { Landscape } \\
\text { (Mountains) }\end{array}$ \\
\hline 222 & W1995.030.224 & $\begin{array}{l}\text { From the door of Mr. } \\
\text { Wilkinsons Hotel }\end{array}$ & $\begin{array}{l}\text { August 12, } \\
1872\end{array}$ & $\begin{array}{c}\text { WVRHC } \\
\text { Description Page }\end{array}$ & Unknown & No Location on Drawing & $\begin{array}{l}\text { Architecture } \\
\text { (Residential } \\
\text { Buildings), } \\
\text { Children, } \\
\text { Landscape } \\
\text { (Fences), } \\
\text { Landscape } \\
\text { (Mountains), } \\
\text { Men, Women }\end{array}$ \\
\hline 223 & W1995.030.367 & $\begin{array}{l}\text { Gay Head (Massachusetts) } \\
\text { Simon Johnson Aged } 65 \text { June } \\
\text { 1st } 1859\end{array}$ & June 1,1859 & On Drawing & Gay Head, MA & On Drawing & Men \\
\hline 224 & W1995.030.373 & Gay Head. May 30th..1859 & May 30,1859 & On Drawing & Gay Head, MA & On Drawing & Landscape \\
\hline 225 & W1995.030.307 & $\begin{array}{l}\text { Giants aisle Saturday 14-- } \\
\text { Wyer's Cave }\end{array}$ & $\begin{array}{l}\text { October } 14 \\
1853\end{array}$ & On Drawing & Weyer's Cave, VA & On Drawing & $\begin{array}{l}\text { Landscape } \\
\text { (Caves) }\end{array}$ \\
\hline 226 & W1995.030.011 & $\begin{array}{l}\text { Glen Ellis- } 5 \text { miles from Glen } \\
\text { House Mountain }\end{array}$ & $\begin{array}{l}\text { September } \\
21,1845\end{array}$ & $\begin{array}{c}\text { WVRHC } \\
\text { Description Page }\end{array}$ & White Mountains, NH & $\frac{\text { http://whitemountainhistor }}{\text { y.org/Glen_House.html }}$ & $\begin{array}{l}\text { Landscape } \\
\text { (Mountains), } \\
\text { Landscape } \\
\text { (Waterfalls) }\end{array}$ \\
\hline 227 & W1995.030.394pg13 & Glen House & 1859 & On Drawing & White Mountains, NH & $\begin{array}{l}\text { http://whitemountainhistor } \\
\text { y.org/Glen_House.html }\end{array}$ & Animals (Wild) \\
\hline
\end{tabular}




\begin{tabular}{|c|c|c|c|c|c|c|c|}
\hline Number & Accession Number & Title & Date & $\begin{array}{l}\text { Source of Date } \\
\text { Information }\end{array}$ & Location & $\begin{array}{l}\text { Source of Location } \\
\text { Information }\end{array}$ & Themes \\
\hline 228 & W1995.030.392pg11 & $\begin{array}{l}\text { Going for Clams. April } \\
\text { 28..1887 Powelton. Wm } \\
\text { James Mason. May 2nd.. }\end{array}$ & $\begin{array}{c}\text { April 28, } \\
\text { 1887/ May } 2\end{array}$ & On Drawing & Accomack County, VA & $\begin{array}{c}\text { Cecil Eby, "Porte } \\
\text { Crayon": The Life of } \\
\text { David Hunter Strother, } \\
\text { (Chapel Hill, NC: } \\
\text { University of North } \\
\text { Carolina Press, 1960), } \\
\text { 204. }\end{array}$ & Men at Work \\
\hline 229 & W1995.030.179 & $\begin{array}{l}\text { Gold Hill Work near Salisbury } \\
\text { North Carolina }\end{array}$ & Unknown & $\begin{array}{l}\text { No Date on } \\
\text { Drawing }\end{array}$ & Salisbury, NC & On Drawing & $\begin{array}{l}\text { Architecture } \\
\text { (Towns), } \\
\text { Industry, Men } \\
\text { at Work }\end{array}$ \\
\hline 230 & W1995.030.393pg31b & Gouge. Yancey Co N.C. & Unknown & $\begin{array}{l}\text { No Date on } \\
\text { Drawing }\end{array}$ & Yancey County, NC & On Drawing & Men \\
\hline 231 & W1995.030.312 & $\begin{array}{l}\text { Great Oak of Purrgoteague } \\
\text { Accomac Co: Va.. May } \\
\text { 1st..1887. }\end{array}$ & May 1,1887 & On Drawing & Accomack County, VA & On Drawing & $\begin{array}{l}\text { African } \\
\text { Americans, } \\
\text { Animals } \\
\text { (Domestic), } \\
\text { Architecture } \\
\text { (Residential } \\
\text { Buildings), } \\
\text { Children, } \\
\text { Landscape } \\
\text { (Fences), Men } \\
\text { at Work, } \\
\text { Transportation } \\
\text { (Carriages) }\end{array}$ \\
\hline 232 & W1995.030.372 & $\begin{array}{l}\text { Great Pond Cohasset June } \\
\text { 13th..1859 }\end{array}$ & June 13,1859 & On Drawing & Cohasset, MA & On Drawing & $\begin{array}{l}\text { Children, } \\
\text { Landscape } \\
\text { (Lakes), } \\
\text { Transportation } \\
\text { (Boats) }\end{array}$ \\
\hline
\end{tabular}




\begin{tabular}{|c|c|c|c|c|c|c|c|}
\hline Number & Accession Number & Title & Date & $\begin{array}{l}\text { Source of Date } \\
\text { Information }\end{array}$ & Location & $\begin{array}{l}\text { Source of Location } \\
\text { Information }\end{array}$ & Themes \\
\hline 233 & W1995.030.045 & $\begin{array}{l}\text { Guard Room- Harper's } \\
\text { Ferry.Ossawottomie Brown- } \\
\text { Aaron Stephens wounded- }\end{array}$ & $\begin{array}{l}\text { October } 18 \\
1859\end{array}$ & On Drawing & $\begin{array}{l}\text { Harpers Ferry, VA } \\
\text { (WV) }\end{array}$ & On Drawing & $\begin{array}{l}\text { Harper's Ferry } \\
\text { Raid Trial, } \\
\text { Men, Military }\end{array}$ \\
\hline 234 & W1995.030.168 & $\begin{array}{l}\text { Guilford Court House } \\
\text { Martinsville April 24, } 1856\end{array}$ & $\begin{array}{l}\text { April 24, } \\
1856\end{array}$ & On Drawing & Martinsville, NC & On Drawing & $\begin{array}{l}\text { Architecture } \\
\text { (Towns), } \\
\text { Landscape }\end{array}$ \\
\hline 235 & W1995.030.394pg11 & Haddock. June 12th 1859. & June 12,1859 & On Drawing & Unknown & No Location on Drawing & Animals (Wild) \\
\hline 236 & W1995.030.060 & $\begin{array}{l}\text { Halt at ClarksburgMcClellan's } \\
\text { Staff }\end{array}$ & $\begin{array}{l}\text { September } \\
12,1862\end{array}$ & On Drawing & Clarksburg, MD & On Drawing & $\begin{array}{l}\text { Animals } \\
\text { (Domestic), } \\
\text { Architecture } \\
\text { (Residential } \\
\text { Buildings), } \\
\text { Men, Military }\end{array}$ \\
\hline 237 & W1995.030.387pg45 & $\begin{array}{l}\text { Hannah. grand daughter of } \\
\text { Henry Church. July 22nd } \\
1858 \text {. }\end{array}$ & July 22,1858 & On Drawing & Unknown & No Location on Drawing & $\begin{array}{l}\text { Decorative } \\
\text { Arts, Women }\end{array}$ \\
\hline 238 & W1995.030.047 & $\begin{array}{l}\text { Harper's Ferry. A Son of John } \\
\text { Brown- alias Ossawottomie } \\
\text { Brown of Kansas notoriety. }\end{array}$ & $\begin{array}{l}\text { October } 18 \\
1859\end{array}$ & On Drawing & $\begin{array}{l}\text { Harpers Ferry, VA } \\
\text { (WV) }\end{array}$ & On Drawing & $\begin{array}{l}\text { Harper's Ferry } \\
\text { Raid Trial, } \\
\text { Men }\end{array}$ \\
\hline 239 & W1995.030.232a & $\begin{array}{l}\text { Harris Hetterick Randolf Co. } \\
\text { Va. }\end{array}$ & $\begin{array}{l}\text { September, } \\
\quad 1860\end{array}$ & On Drawing & $\begin{array}{c}\text { Randolph County, VA } \\
\text { (WV) }\end{array}$ & On Drawing & Children \\
\hline
\end{tabular}




\begin{tabular}{|c|c|c|c|c|c|c|c|}
\hline Number & Accession Number & Title & Date & $\begin{array}{l}\text { Source of Date } \\
\text { Information }\end{array}$ & Location & $\begin{array}{l}\text { Source of Location } \\
\text { Information }\end{array}$ & Themes \\
\hline 240 & W1995.030.177 & $\begin{array}{l}\text { Hauling in the Seine, } \\
\text { Belvedere (NC) Fishery }\end{array}$ & April 9, 1856 & On Drawing & Belvedere, NC & On Drawing & $\begin{array}{l}\text { African } \\
\text { Americans, } \\
\text { Animals } \\
\text { (Domestic), } \\
\text { Architecture, } \\
\text { Men at Work, } \\
\text { Transportation } \\
\text { (Boats) }\end{array}$ \\
\hline 241 & W1995.030.392pg3a & Havanna May 1879 & May 1879 & On Drawing & Havana, Cuba & On Drawing & $\begin{array}{l}\text { Animals } \\
\text { (Domestic), } \\
\text { Men, } \\
\text { Transportation } \\
\text { (Carriages) }\end{array}$ \\
\hline 242 & W1995.030.392pg1a & Havanna May 3rd 1879. & May 3, 1879 & On Drawing & Havana, Cuba & On Drawing & $\begin{array}{l}\text { Animals } \\
\text { (Domestic), } \\
\text { Men at Work, } \\
\text { Transportation } \\
\text { (Carriages) }\end{array}$ \\
\hline 243 & W1995.030.166 & $\begin{array}{l}\text { Heading Herring--Leary's } \\
\text { (NC) April 8, } 1856\end{array}$ & April 8, 1856 & On Drawing & North Carolina & On Drawing & $\begin{array}{l}\text { African } \\
\text { Americans, } \\
\text { Animals } \\
\text { (Wild), } \\
\text { Women at } \\
\text { Work }\end{array}$ \\
\hline 244 & W1995.030.388pg20 & $\begin{array}{l}\text { Heiney Whistler Martinsburg } \\
1838 .\end{array}$ & 1838 & On Drawing & Martinsburg, VA (WV) & On Drawing & Men at Leisure \\
\hline
\end{tabular}




\begin{tabular}{|c|c|c|c|c|c|c|c|}
\hline Number & Accession Number & Title & Date & $\begin{array}{l}\text { Source of Date } \\
\text { Information }\end{array}$ & Location & $\begin{array}{l}\text { Source of Location } \\
\text { Information }\end{array}$ & Themes \\
\hline 245 & W1995.030.014 & $\begin{array}{l}\text { Henri Herbell of Munthausen- } \\
\text { Hesse Darmstadt and his dogs }\end{array}$ & 1850s & $\begin{array}{l}\text { On Drawing } \\
\text { (Exact Date } \\
\text { Unclear) }\end{array}$ & $\begin{array}{l}\text { Berkeley Springs, VA } \\
\text { (WV) }\end{array}$ & $\begin{array}{l}\text { Strother, "The Bear and } \\
\text { the Basket Maker," } \\
\text { Harpers New Monthly } \\
\text { Magazine, June } 1856 .\end{array}$ & $\begin{array}{l}\text { Animals } \\
\text { Domestic, Men }\end{array}$ \\
\hline 246 & W1995.030.252 & $\begin{array}{l}\text { Henry Church's house. } 3 \text { miles } \\
\text { beyond Burton. Balte..\& Ohio } \\
\text { R. Road }\end{array}$ & Unknown & $\begin{array}{l}\text { No Date on } \\
\text { Drawing }\end{array}$ & Burton, $\mathrm{OH}$ & On Drawing & $\begin{array}{l}\text { Animals } \\
\text { (Domestic), } \\
\text { Architecture } \\
\text { (Residential } \\
\text { Buildings), } \\
\text { Women }\end{array}$ \\
\hline 247 & W1995.030.084 & Henry Elliot Near Bologna & $\begin{array}{l}\text { April 21, } \\
\quad 1843\end{array}$ & On Drawing & Bologna, Italy & On Drawing & Men \\
\hline 248 & W1995.030.192 & Holston Springs & Unknown & $\begin{array}{l}\text { No Date on } \\
\text { Drawing }\end{array}$ & Holston Springs, VA & On Drawing & $\begin{array}{l}\text { Architecture } \\
\text { (Institutional } \\
\text { Buildings) }\end{array}$ \\
\hline 249 & W1995.030.029 & Hooper's Saloon & $\begin{array}{l}\text { December } 18, \\
1860\end{array}$ & On Drawing & Unknown & No Location on Drawing & Animals, Men \\
\hline 250 & W1995.030.268 & Hooper's-Sept..15th..1859 & $\begin{array}{l}\text { September } \\
15,1859\end{array}$ & On Drawing & Unknown & No Location on Drawing & Men \\
\hline 251 & W1995.030.387pg36a & $\begin{array}{l}\text { Horse Camp Road. Dismal } \\
\text { Swamp, Va. March 20th } 1856 .\end{array}$ & $\begin{array}{l}\text { March 20, } \\
\quad 1856\end{array}$ & On Drawing & Dismal Swamp, VA & On Drawing & $\begin{array}{l}\text { Animals } \\
\text { (Domestic), } \\
\text { Architecture } \\
\text { (Residential } \\
\text { Buildings), } \\
\text { Landscape } \\
\text { (Roads), Men } \\
\text { at Work, } \\
\text { Transportation } \\
\text { (Carriages) }\end{array}$ \\
\hline
\end{tabular}




\begin{tabular}{|c|c|c|c|c|c|c|c|}
\hline Number & Accession Number & Title & Date & $\begin{array}{l}\text { Source of Date } \\
\text { Information }\end{array}$ & Location & $\begin{array}{l}\text { Source of Location } \\
\text { Information }\end{array}$ & Themes \\
\hline 252 & W1995.030.176 & Horse Canyon Road & $\begin{array}{l}\text { March 20, } \\
1856\end{array}$ & On Drawing & Unknown & No Location on Drawing & $\begin{array}{l}\text { African } \\
\text { Americans, } \\
\text { Animals } \\
\text { (Domestic), } \\
\text { Men at Work, } \\
\text { Landscape } \\
\text { (Roads), } \\
\text { Transportation } \\
\text { (Carriages) }\end{array}$ \\
\hline 253 & W1995.030.394pg21b & $\begin{array}{l}\text { Hoyt of Boston Old John } \\
\text { Brown's Lawyer }\end{array}$ & October 1859 & On Drawing & $\begin{array}{l}\text { Charles Town, VA } \\
\text { (WV) }\end{array}$ & & $\begin{array}{l}\text { Decorative } \\
\text { Arts, Harper's } \\
\text { Ferry Raid } \\
\text { Trial, Men }\end{array}$ \\
\hline 254 & W1995.030.377 & $\begin{array}{l}\text { Hut on the Winston Farm } \\
\text { (Berkeley Co.) June } 1851\end{array}$ & June 1851 & On Drawing & $\begin{array}{l}\text { Berkeley County, VA } \\
\text { (WV) }\end{array}$ & On Drawing & $\begin{array}{l}\text { Architecture } \\
\text { (Residential } \\
\text { Buildings) }\end{array}$ \\
\hline 255 & W1995.030.387pg34a & $\begin{array}{l}\text { Ice Mountain - Hampshire Co. } \\
\text { Va. Oct. 5th }\end{array}$ & Unknown & $\begin{array}{l}\text { No Date on } \\
\text { Drawing }\end{array}$ & $\begin{array}{l}\text { Hampshire County, VA } \\
\text { (WV) }\end{array}$ & On Drawing & $\begin{array}{l}\text { Landscape } \\
\text { (Mountains) }\end{array}$ \\
\hline 256 & W1995.030.100 & $\begin{array}{l}\text { Il Paradisino near } \\
\text { Vallombrosa }\end{array}$ & May 1842 & On Drawing & Vallombrosa, Italy & On Drawing & $\begin{array}{l}\text { Architecture, } \\
\text { Landscape } \\
\text { (Mountains) }\end{array}$ \\
\hline 257 & W1995.030.393pg23b & Illegible title - 3 caricatures & July 1857 & $\begin{array}{c}\text { WVRHC } \\
\text { Description Page }\end{array}$ & Unknown & No Location on Drawing & Men \\
\hline 258 & W1995.030.217 & $\begin{array}{l}\text { Illegible title Point (?) high } \\
\text { BC (landscape with sailboat) }\end{array}$ & April 1875 & $\begin{array}{c}\text { WVRHC } \\
\text { Description Page }\end{array}$ & Unknown & No Location on Drawing & $\begin{array}{l}\text { Architecture } \\
\text { (Residential } \\
\text { Buildings), } \\
\text { Landscape } \\
\text { (Rivers), } \\
\text { Transportation } \\
\text { (Boats) }\end{array}$ \\
\hline
\end{tabular}




\begin{tabular}{|c|c|c|c|c|c|c|c|}
\hline Number & Accession Number & Title & Date & $\begin{array}{l}\text { Source of Date } \\
\text { Information }\end{array}$ & Location & $\begin{array}{l}\text { Source of Location } \\
\text { Information }\end{array}$ & Themes \\
\hline 259 & W1995.030.392pg5 & In Memoriam Feby 9th 1887 & $\begin{array}{c}\text { February } 9 \\
1887\end{array}$ & On Drawing & Unknown & No Location on Drawing & $\begin{array}{l}\text { Architecture } \\
\text { (Residential } \\
\text { Buildings) }\end{array}$ \\
\hline 260 & W1995.030.181 & Indian Head Fisheries & May 7,1859 & On Drawing & Indian Head, MD & $\begin{array}{c}\text { Jessie Poesch, "Eight } \\
\text { Drawings by David } \\
\text { Hunter Strother in the } \\
\text { Walters Collection," The } \\
\text { Journal of the Walters Art } \\
\text { Gallery 52/53 } \\
\text { (1994/1995): } 75 .\end{array}$ & Men at Work \\
\hline 261 & W1995.030.160 & Indian Head Fishery Sunday & May 1859 & On Drawing & Indian Head, MD & $\begin{array}{c}\text { Jessie Poesch, "Eight } \\
\text { Drawings by David } \\
\text { Hunter Strother in the } \\
\text { Walters Collection," The } \\
\text { Journal of the Walters Art } \\
\text { Gallery 52/53 } \\
\text { (1994/1995): } 75 .\end{array}$ & $\begin{array}{l}\text { African } \\
\text { Americans, } \\
\text { Men at Leisure, } \\
\text { Women at } \\
\text { Leisure }\end{array}$ \\
\hline 262 & W1995.030.163 & $\begin{array}{l}\text { Indianhead fishery May 5, } \\
1859\end{array}$ & May 5,1859 & On Drawing & Indian Head, MD & $\begin{array}{c}\text { Jessie Poesch, "Eight } \\
\text { Drawings by David } \\
\text { Hunter Strother in the } \\
\text { Walters Collection," The } \\
\text { Journal of the Walters Art } \\
\text { Gallery 52/53 } \\
\text { (1994/1995): } 75 .\end{array}$ & $\begin{array}{l}\text { African } \\
\text { Americans, } \\
\text { Landscape } \\
\text { (Lakes), Men } \\
\text { at Leisure, } \\
\text { Transportation } \\
\text { (Boats) }\end{array}$ \\
\hline 263 & W1995.030.387pg35a & $\begin{array}{l}\text { Interior View Salt Works. } \\
\text { Nov. 19th } 1856\end{array}$ & $\begin{array}{l}\text { November } \\
19,1856\end{array}$ & On Drawing & Unknown & No Location on Drawing & $\begin{array}{l}\text { Architecture, } \\
\text { Industry, Men } \\
\text { at Work }\end{array}$ \\
\hline
\end{tabular}




\begin{tabular}{|c|c|c|c|c|c|c|c|}
\hline Number & Accession Number & Title & Date & $\begin{array}{l}\text { Source of Date } \\
\text { Information }\end{array}$ & Location & $\begin{array}{l}\text { Source of Location } \\
\text { Information }\end{array}$ & Themes \\
\hline 264 & W1995.030.388pg21 & $\begin{array}{l}\text { Invalids. 1845. Winchester } \\
\text { Virginia }\end{array}$ & 1845 & On Drawing & Winchester, VA & On Drawing & $\begin{array}{l}\text { Decorative } \\
\text { Arts, Men at } \\
\text { Leisure }\end{array}$ \\
\hline 265 & W1995.030.283 & $\begin{array}{l}\text { Ironworks Chatenoga [sic] } \\
\text { Tenn }\end{array}$ & $\begin{array}{l}\text { October 26, } \\
1859\end{array}$ & $\begin{array}{c}\text { WVRHC } \\
\text { Description Page }\end{array}$ & Chattanooga, TN & On Drawing & $\begin{array}{l}\text { Architecture, } \\
\text { Industry, } \\
\text { Landscape } \\
\text { (Mountains), } \\
\text { Landscape } \\
\text { (Rivers), Men, } \\
\text { Transportation } \\
\text { (Boats) }\end{array}$ \\
\hline 266 & W1995.030.364 & $\begin{array}{l}\text { J.B. Gardner. Waggon Master } \\
\text { Ore Knob Guilford, NC July } 3 \\
1874\end{array}$ & July 3,1874 & On Drawing & Guilford, NC & On Drawing & Men \\
\hline 267 & W1995.030.393pg26b & J.W. Ehringer & Unknown & $\begin{array}{l}\text { No Date on } \\
\text { Drawing }\end{array}$ & Unknown & No Location on Drawing & Men \\
\hline 268 & W1995.030.393pg22a & Jackson May 4th 1858. & May 4, 1858 & On Drawing & Unknown & No Location on Drawing & $\begin{array}{l}\text { African } \\
\text { Americans, } \\
\text { Men }\end{array}$ \\
\hline 269 & W1995.030.394pg15 & Jackson, White Mountain & June 26,1859 & On Drawing & White Mountains, NH & On Drawing & $\begin{array}{l}\text { Decorative } \\
\text { Arts, Men at } \\
\text { Leisure }\end{array}$ \\
\hline 270 & W1995.030.227 & Jacob Miller Jany 3rd 1872 & $\begin{array}{c}\text { January } 3, \\
1872\end{array}$ & On Drawing & Unknown & No Location on Drawing & Children \\
\hline
\end{tabular}




\begin{tabular}{|c|c|c|c|c|c|c|c|}
\hline Number & Accession Number & Title & Date & $\begin{array}{l}\text { Source of Date } \\
\text { Information }\end{array}$ & Location & $\begin{array}{l}\text { Source of Location } \\
\text { Information }\end{array}$ & Themes \\
\hline 271 & W1995.030.391pg18 & $\begin{array}{l}\text { Jacob Vinsonbeller } \\
\text { Martinsburg } 1846 .\end{array}$ & 1846 & On Drawing & Martinsburg, VA (WV) & On Drawing & Men at Work \\
\hline 272 & W1995.030.269 & $\begin{array}{l}\text { James C. Steele-Cooper Indian } \\
\text { Head Fishery }\end{array}$ & May 6,1859 & On Drawing & Indian Head, MD & $\begin{array}{c}\text { Jessie Poesch, "Eight } \\
\text { Drawings by David } \\
\text { Hunter Strother in the } \\
\text { Walters Collection," The } \\
\text { Journal of the Walters Art } \\
\text { Gallery 52/53 } \\
\text { (1994/1995): } 75 .\end{array}$ & $\begin{array}{l}\text { Industry, Men } \\
\text { at Work }\end{array}$ \\
\hline 273 & W1995.030.257 & $\begin{array}{l}\text { James River \& Kanawha } \\
\text { Canal November } 1856\end{array}$ & $\begin{array}{l}\text { November } \\
1856\end{array}$ & On Drawing & Unknown & No Location on Drawing & $\begin{array}{l}\text { African } \\
\text { Americans, } \\
\text { Men at Work }\end{array}$ \\
\hline 274 & W1995.030.262 & Jany 21 st...1876 & $\begin{array}{l}\text { January 21, } \\
\quad 1876\end{array}$ & On Drawing & Unknown & No Location on Drawing & Children \\
\hline 275 & W1995.030.393pg11b & Je ne comprend rien- & $\begin{array}{l}\text { March 26, } \\
\quad 1857\end{array}$ & $\begin{array}{c}\text { WVRHC } \\
\text { Description Page }\end{array}$ & Unknown & No Location on Drawing & Men at Work \\
\hline 276 & W1995.030.241 & $\begin{array}{l}\text { Jim Pierce Dismal Swamp } \\
1856\end{array}$ & 1856 & On Drawing & Dismal Swamp, VA & On Drawing & $\begin{array}{l}\text { African } \\
\text { Americans, } \\
\text { Men at Work }\end{array}$ \\
\hline 277 & W1995.030.390pg24 & $\begin{array}{l}\text { Joe Cranes Barn, Jefferson Co. } \\
\text { Virginia } 1847.1850\end{array}$ & $1847 / 1850$ & On Drawing & $\begin{array}{l}\text { Jefferson County, VA } \\
\text { (WV) }\end{array}$ & On Drawing & $\begin{array}{l}\text { Animals } \\
\text { (Domestic), } \\
\text { Architecture }\end{array}$ \\
\hline 278 & W1995.030.394pg20b & John Brown & $\begin{array}{l}\text { December 2, } \\
1859\end{array}$ & On Drawing & $\begin{array}{l}\text { Charles Town, VA } \\
\text { (WV) }\end{array}$ & See Number 281 & $\begin{array}{l}\text { Harper's Ferry } \\
\text { Raid Trial, } \\
\text { Men }\end{array}$ \\
\hline 279 & W1995.030.046 & $\begin{array}{l}\text { John Brown in Court, } \\
\text { Charleston, Va. }\end{array}$ & $\begin{array}{l}\text { October 27, } \\
1859\end{array}$ & On Drawing & $\begin{array}{l}\text { Charles Town, VA } \\
\text { (WV) }\end{array}$ & On Drawing & $\begin{array}{l}\text { Harper's Ferry } \\
\text { Raid Trial, } \\
\text { Men }\end{array}$ \\
\hline
\end{tabular}




\begin{tabular}{|c|c|c|c|c|c|c|c|}
\hline Number & Accession Number & Title & Date & $\begin{array}{l}\text { Source of Date } \\
\text { Information }\end{array}$ & Location & $\begin{array}{l}\text { Source of Location } \\
\text { Information }\end{array}$ & Themes \\
\hline 280 & W1995.030.394pg29 & $\begin{array}{l}\text { John Brown on Trial, Oct. 26, } \\
\text { 1859, Charleston, Va. }\end{array}$ & $\begin{array}{l}\text { October } 26 \\
1859\end{array}$ & On Drawing & $\begin{array}{l}\text { Charles Town, VA } \\
\text { (WV) }\end{array}$ & On Drawing & $\begin{array}{l}\text { Harper's Ferry } \\
\text { Raid Trial, } \\
\text { Men }\end{array}$ \\
\hline 281 & W1995.030.044 & John Brown, Charleston, Va. & $\begin{array}{l}\text { December } 2 \\
\quad 1859\end{array}$ & On Drawing & $\begin{array}{l}\text { Charles Town, VA } \\
\text { (WV) }\end{array}$ & On Drawing & $\begin{array}{l}\text { Harper's Ferry } \\
\text { Raid Trial, } \\
\text { Men }\end{array}$ \\
\hline 282 & W1995.030.394pg30 & $\begin{array}{l}\text { John Brown, Nov. 1859/crude } \\
\text { pencil drawing of a child (not } \\
\text { Strother's hand) }\end{array}$ & $\begin{array}{c}\text { November } \\
1859\end{array}$ & On Drawing & $\begin{array}{l}\text { Charles Town, VA } \\
\text { (WV) }\end{array}$ & See Numbers 279 to 283 & $\begin{array}{l}\text { Children, } \\
\text { Harper's Ferry } \\
\text { Raid Trial, } \\
\text { Men }\end{array}$ \\
\hline 283 & W1995.030.394pg33 & $\begin{array}{l}\text { John Brown's last words on } \\
\text { the gallows Dec: } 2 \text { nd } 1859\end{array}$ & $\begin{array}{l}\text { December } 2 \\
\quad 1859\end{array}$ & On Drawing & $\begin{array}{l}\text { Charles Town, VA } \\
\text { (WV) }\end{array}$ & See Number 281 & $\begin{array}{l}\text { Harper's Ferry } \\
\text { Raid Trial, } \\
\text { Men }\end{array}$ \\
\hline 284 & W1995.030.023 & $\begin{array}{l}\text { John Cassin, Berney Wolff \& } \\
\text { Strother. Aaron Armantrout's }\end{array}$ & July 1,1854 & On Drawing & $\begin{array}{l}\text { Randolph County, VA } \\
\text { (WV) }\end{array}$ & See Number 15 & $\begin{array}{l}\text { Architecture } \\
\text { (Residential } \\
\text { Buildings), } \\
\text { Decorative } \\
\text { Arts }\end{array}$ \\
\hline 285 & W1995.030.255 & $\begin{array}{l}\text { John Cassin--ascending The } \\
\text { Blackwater-- }\end{array}$ & Unknown & $\begin{array}{l}\text { No Date on } \\
\text { Drawing }\end{array}$ & $\begin{array}{l}\text { Canaan Valley, VA } \\
\text { (WV) }\end{array}$ & On Drawing & $\begin{array}{l}\text { Landscape } \\
\text { (Mountains), } \\
\text { Men in } \\
\text { Wilderness }\end{array}$ \\
\hline 286 & W1995.030.369 & $\begin{array}{l}\text { John Hittson Denver Aug } 10 \\
\text { 1873.. }\end{array}$ & $\begin{array}{l}\text { August } 10 \\
1873\end{array}$ & On Drawing & Denver, Colorado & On Drawing & Men \\
\hline
\end{tabular}




\begin{tabular}{|c|c|c|c|c|c|c|c|}
\hline Number & Accession Number & Title & Date & $\begin{array}{l}\text { Source of Date } \\
\text { Information }\end{array}$ & Location & $\begin{array}{l}\text { Source of Location } \\
\text { Information }\end{array}$ & Themes \\
\hline 287 & W1995.030.391pg17 & $\begin{array}{l}\text { John Jamison, Agent } \\
\text { Martinsburg, Va. } 1845\end{array}$ & 1845 & On Drawing & Martinsburg, VA (WV) & On Drawing & $\begin{array}{l}\text { Decorative } \\
\text { Arts, Men }\end{array}$ \\
\hline 288 & W1995.030.387pg4 & $\begin{array}{l}\text { John Selden's Barn. Cave } \\
\text { farm Jefferson Co: Virginia } \\
1859\end{array}$ & 1859 & On Drawing & $\begin{array}{l}\text { Jefferson County, VA } \\
\text { (WV) }\end{array}$ & On Drawing & $\begin{array}{l}\text { African } \\
\text { Americans, } \\
\text { Architecture, } \\
\text { Men at Work, } \\
\text { Transportation } \\
\text { (Carriages) }\end{array}$ \\
\hline 289 & W1995.030.395pg9 & $\begin{array}{l}\text { John Will. Berkeley Springs } \\
\text { West Va Octo 3rd } 1873 .\end{array}$ & $\begin{array}{l}\text { October 3, } \\
1873\end{array}$ & On Drawing & $\begin{array}{l}\text { Berkeley Springs, VA } \\
\text { (WV) }\end{array}$ & On Drawing & Children \\
\hline 290 & W1995.030.387pg23a & $\begin{array}{l}\text { Johnson Wilson Is (sic) these } \\
\text { saddle bags full of money? }\end{array}$ & Unknown & $\begin{array}{l}\text { Date on Drawing } \\
\text { Unclear }\end{array}$ & Unknown & No Location on Drawing & Children \\
\hline 291 & W1995.030.387pg44 & $\begin{array}{l}\text { July } 22 \text { nd } 1858 \text { Hannah } \\
\text { Church going on } 104 \text { years of } \\
\text { age }\end{array}$ & July 22,1858 & On Drawing & Unknown & No Location on Drawing & $\begin{array}{l}\text { Decorative } \\
\text { Arts, Women }\end{array}$ \\
\hline 292 & W1995.030.387pg43 & $\begin{array}{l}\text { July 22nd } 1858 . \text { Henry } \\
\text { Church. Aged } 108\end{array}$ & July 22,1858 & On Drawing & Unknown & No Location on Drawing & $\begin{array}{l}\text { Decorative } \\
\text { Arts, Men }\end{array}$ \\
\hline 293 & W1995.030.248b & $\begin{array}{l}\text { Junius Lenoire Baker Adelaide } \\
\text { Dec. 5th 1856. Pap. I want } \\
\text { some gravy }\end{array}$ & $\begin{array}{l}\text { December } 5 \text {, } \\
\quad 1856\end{array}$ & On Drawing & Unknown & No Location on Drawing & Children \\
\hline 294 & W1995.030.393pg20b & Kanawha & $\begin{array}{c}\text { December 16, } \\
1858 / \\
\text { October } 1858\end{array}$ & $\begin{array}{c}\text { WVRHC } \\
\text { Description Page }\end{array}$ & $\begin{array}{c}\text { Kanawha County, VA } \\
\text { (WV) }\end{array}$ & On Drawing & $\begin{array}{l}\text { Decorative } \\
\text { Arts, Men at } \\
\text { Leisure }\end{array}$ \\
\hline 295 & W1995.030.393pg25b & Kanawha & Unknown & $\begin{array}{l}\text { No Date on } \\
\text { Drawing }\end{array}$ & $\begin{array}{c}\text { Kanawha County, VA } \\
\text { (WV) }\end{array}$ & On Drawing & $\begin{array}{l}\text { Men at Work, } \\
\text { Transportation } \\
\text { (Boats), } \\
\text { Transportation } \\
\text { (Carriages) }\end{array}$ \\
\hline
\end{tabular}




\begin{tabular}{|c|c|c|c|c|c|c|c|}
\hline Number & Accession Number & Title & Date & $\begin{array}{l}\text { Source of Date } \\
\text { Information }\end{array}$ & Location & $\begin{array}{l}\text { Source of Location } \\
\text { Information }\end{array}$ & Themes \\
\hline 296 & W1995.030.391pg16 & $\begin{array}{l}\text { Keeping Tally Martinsburg } \\
1844 .\end{array}$ & 1844 & On Drawing & Martinsburg, VA (WV) & On Drawing & Children \\
\hline 297 & W1995.030.287 & $\begin{array}{l}\text { Ken Foster. Black Mountains. } \\
\text { East Tenn. }\end{array}$ & $\begin{array}{c}\text { December } 9 \\
1856\end{array}$ & On Drawing & Black Mountains, TN & On Drawing & Men \\
\hline 298 & W1995.030.394pg7 & $\begin{array}{l}\text { King Crab \& Star on Gay head } \\
\text { beach }\end{array}$ & May 30,1859 & On Drawing & Gay Head, MA & See Number 223 & Animals \\
\hline 299 & W1995.030.393pg34a & Knoxville Tenne. Jany 1857. & January 1857 & On Drawing & Knoxville, TN & On Drawing & Men, Women \\
\hline 300 & W1995.030.042 & La Brunette au Bain & $\begin{array}{c}\text { September } 1 \\
1887\end{array}$ & On Drawing & Unknown & No Location on Drawing & $\begin{array}{l}\text { Architecture, } \\
\text { Women }\end{array}$ \\
\hline 301 & W1995.030.148 & La Isla de Trinidad & Unknown & $\begin{array}{l}\text { No Date on } \\
\text { Drawing }\end{array}$ & Trinidad & On Drawing & Women \\
\hline 302 & W1995.030.149 & La Isla de Trinidad No. 2 & Unknown & $\begin{array}{l}\text { No Date on } \\
\text { Drawing }\end{array}$ & Trinidad & On Drawing & $\begin{array}{l}\text { Landscape } \\
\text { (Mountains), } \\
\text { Women }\end{array}$ \\
\hline 303 & W1995.030.392pg4 & $\begin{array}{l}\text { La Piedad - Mexico, 188- M } \\
\text { Lewzarder }\end{array}$ & Unknown & $\begin{array}{l}\text { No Date on } \\
\text { Drawing }\end{array}$ & Mexico City, Mexico & On Drawing & $\begin{array}{l}\text { Animals } \\
\text { (Domestic), } \\
\text { Men, Women }\end{array}$ \\
\hline 304 & W1995.030.135 & $\begin{array}{l}\text { Landing at Memphis Navy } \\
\text { Yard. March } 28\end{array}$ & Unknown & $\begin{array}{l}\text { No Date on } \\
\text { Drawing }\end{array}$ & Memphis, TN & On Drawing & $\begin{array}{l}\text { Landscape } \\
\text { (Rivers), Men } \\
\text { at Work, } \\
\text { Transportation } \\
\text { (Boats) }\end{array}$ \\
\hline 305 & W1995.030.155 & $\begin{array}{l}\text { Lawrence R.R. New England } \\
1859 \text {. Lowell. June } 24,1859 . \\
\text { (3 sets of sketches) }\end{array}$ & June 24, 1859 & On Drawing & New England & On Drawing & $\begin{array}{l}\text { Men at Leisure, } \\
\text { Women }\end{array}$ \\
\hline 306 & W1995.030.291 & $\begin{array}{l}\text { Leonard Moler Guide to } \\
\text { Weyer's Cave at } 13 \text { years }\end{array}$ & 1853 & On Drawing & Weyer's Cave, VA & On Drawing & Men at Work \\
\hline
\end{tabular}




\begin{tabular}{|c|c|c|c|c|c|c|c|}
\hline Number & Accession Number & Title & Date & $\begin{array}{l}\text { Source of Date } \\
\text { Information }\end{array}$ & Location & $\begin{array}{l}\text { Source of Location } \\
\text { Information }\end{array}$ & Themes \\
\hline 307 & W1995.030.264 & $\begin{array}{l}\text { Levi Wolff (boy with bull and } \\
\text { horse) }\end{array}$ & Unknown & $\begin{array}{l}\text { No Date on } \\
\text { Drawing }\end{array}$ & Unknown & No Location on Drawing & $\begin{array}{l}\text { Animals } \\
\text { (Domestic), } \\
\text { Men at Work }\end{array}$ \\
\hline 308 & W1995.012.001 & Lighting of the Shrine & 1842 & $\begin{array}{c}\text { WVRHC } \\
\text { Description Page }\end{array}$ & Subiaco, Italy & $\begin{array}{c}\text { John A. Cuthbert and } \\
\text { Jessie Poesch, "David } \\
\text { Hunter Strother: "One of } \\
\text { the Best Draughtsman the } \\
\text { Country Possesses," } \\
\text { (Morgantown, WV: West } \\
\text { Virginia University Press, } \\
\text { 1997), 20. }\end{array}$ & $\begin{array}{l}\text { Animals } \\
\text { (Domestic), } \\
\text { Children, } \\
\text { Landscape } \\
\text { (Mountains), } \\
\text { Men, Religious } \\
\text { Shrines, } \\
\text { Women }\end{array}$ \\
\hline 309 & W1995.030.387pg24b & Listeners & $\begin{array}{l}\text { March 27, } \\
\quad 1857\end{array}$ & On Drawing & Unknown & No Location on Drawing & Men at Leisure \\
\hline 310 & W1995.030.130 & $\begin{array}{l}\text { Live Oak Grove. The Dueling } \\
\text { Ground of New Orleans }\end{array}$ & $\begin{array}{l}\text { December 11, } \\
1873\end{array}$ & $\begin{array}{c}\text { WVRHC } \\
\text { Description Page }\end{array}$ & New Orleans, LA & On Drawing & Landscape \\
\hline 311 & W1995.030.391pg19 & $\begin{array}{l}\text { Lock's Old Stephen. } 1845 . \\
\text { Martinsburg Va. }\end{array}$ & 1845 & On Drawing & Martinsburg, VA (WV) & On Drawing & $\begin{array}{l}\text { African } \\
\text { Americans, } \\
\text { Men at Work }\end{array}$ \\
\hline 312 & W1995.030.332 & $\begin{array}{l}\text { Locust Grove Moorfield } \\
\text { Valley }\end{array}$ & 1848 & $\begin{array}{c}\text { WVRHC } \\
\text { Description Page }\end{array}$ & $\begin{array}{l}\text { Charles Town, VA } \\
\text { (WV) }\end{array}$ & $\begin{array}{l}\text { Cecil Eby, "Porte } \\
\text { Crayon": The Life of } \\
\text { David Hunter Strother, } \\
\text { (Chapel Hill, NC: } \\
\text { University of North } \\
\text { Carolina Press, 1960), } 7 .\end{array}$ & $\begin{array}{l}\text { Animals } \\
\text { (Domestic), } \\
\text { Architecture } \\
\text { (Residential } \\
\text { Buildings), } \\
\text { Men at Work }\end{array}$ \\
\hline 313 & W1995.030.363 & $\begin{array}{l}\text { Look out Boat. May 8th..1859. } \\
\text { Ned Hall \& Dennis Corcoran }\end{array}$ & May 8,1859 & On Drawing & Unknown & No Location on Drawing & $\begin{array}{l}\text { Men at Work, } \\
\text { Transportation } \\
\text { (Boats) }\end{array}$ \\
\hline
\end{tabular}




\begin{tabular}{|c|c|c|c|c|c|c|c|}
\hline Number & Accession Number & Title & Date & $\begin{array}{l}\text { Source of Date } \\
\text { Information }\end{array}$ & Location & $\begin{array}{l}\text { Source of Location } \\
\text { Information }\end{array}$ & Themes \\
\hline 314 & W1995.030.286 & $\begin{array}{l}\text { Look Out Mountain. } \\
\text { Chattanooga Feb. 4th..1857 }\end{array}$ & $\begin{array}{c}\text { February } 4, \\
1857\end{array}$ & On Drawing & Chattanooga, TN & On Drawing & $\begin{array}{l}\text { Architecture } \\
\text { (Towns), } \\
\text { Landscape } \\
\text { (Mountains) }\end{array}$ \\
\hline 315 & W1995.030.289 & $\begin{array}{l}\text { Looking inward from South } \\
\text { end. Natural Tunnel }\end{array}$ & $\begin{array}{l}\text { November } \\
22,1856\end{array}$ & On Drawing & Scott County, VA & See Number 136 & $\begin{array}{l}\text { Landscape } \\
\text { (Caves) }\end{array}$ \\
\hline 316 & W1995.030.050 & $\begin{array}{l}\text { Looking North from New } \\
\text { Market, Va.Genl. Banks } \\
\text { Headquarters }\end{array}$ & May 11,1862 & On Drawing & New Market, VA & On Drawing & $\begin{array}{l}\text { Landscape } \\
\text { (Mountains), } \\
\text { Military }\end{array}$ \\
\hline 317 & W1995.030.055 & $\begin{array}{l}\text { Machine for field } \\
\text { reconnaissances }\end{array}$ & May 1863 & On Drawing & Unknown & No Location on Drawing & $\begin{array}{l}\text { African } \\
\text { Americans, } \\
\text { Landscape } \\
\text { (Mountains), } \\
\text { Men, Military }\end{array}$ \\
\hline 318 & W1995.030.389pg1 & March 1st 1874. & $\begin{array}{c}\text { March 1, } \\
1874\end{array}$ & On Drawing & Unknown & No Location on Drawing & Men at Leisure \\
\hline 319 & W1995.030.389pg2 & March 1st 1874. & $\begin{array}{l}\text { March 1, } \\
1874\end{array}$ & On Drawing & Unknown & No Location on Drawing & $\begin{array}{l}\text { Decorative } \\
\text { Arts, Men }\end{array}$ \\
\hline 320 & W1995.030.251 & $\begin{array}{l}\text { March 21st.. 1859. Clarke Co. } \\
\text { Va. }\end{array}$ & $\begin{array}{l}\text { March 21, } \\
\quad 1859\end{array}$ & On Drawing & Clarke County, VA & On Drawing & Men at Leisure \\
\hline 321 & W1995.030.233 & $\begin{array}{l}\text { March 21st..1859. Clarke Co. } \\
\text { Va. }\end{array}$ & $\begin{array}{l}\text { March 21, } \\
\quad 1859\end{array}$ & On Drawing & Clarke County, VA & On Drawing & Men at Leisure \\
\hline 322 & W1995.030.025 & March 3rd, 1861 & $\begin{array}{c}\text { March 3, } \\
1861\end{array}$ & On Drawing & Unknown & No Location on Drawing & $\begin{array}{l}\text { Decorative } \\
\text { Arts, Men at } \\
\text { Leisure, } \\
\text { Women at } \\
\text { Leisure }\end{array}$ \\
\hline
\end{tabular}




\begin{tabular}{|c|c|c|c|c|c|c|c|}
\hline Number & Accession Number & Title & Date & $\begin{array}{l}\text { Source of Date } \\
\text { Information }\end{array}$ & Location & $\begin{array}{l}\text { Source of Location } \\
\text { Information }\end{array}$ & Themes \\
\hline $\mathbf{3 2 3}$ & W1995.030.376 & March into Canaan & 1845 & $\begin{array}{c}\text { WVRHC } \\
\text { Description Page }\end{array}$ & $\begin{array}{c}\text { Canaan Valley, VA } \\
\text { (WV) }\end{array}$ & $\begin{array}{c}\text { WVRHC Description } \\
\text { Page }\end{array}$ & \\
\hline 324 & W1995.030.137 & Marine Hospital N. Orleans & Unknown & $\begin{array}{l}\text { No Date on } \\
\text { Drawing }\end{array}$ & New Orleans, LA & On Drawing & $\begin{array}{l}\text { Architecture } \\
\text { (Institutional } \\
\text { Buildings), } \\
\text { Landscape } \\
\text { (Rivers), Men } \\
\text { at Work, } \\
\text { Transportation } \\
\text { (Boats) }\end{array}$ \\
\hline 325 & W1995.030.391pg14 & Marinsburg 1845 & 1845 & On Drawing & Martinsburg, VA (WV) & On Drawing & Men \\
\hline 326 & W1995.030.391pg12 & Marinsburg 1845. & 1845 & On Drawing & Martinsburg, VA (WV) & On Drawing & Children \\
\hline 327 & W1995.030.388pg14 & $\begin{array}{l}\text { Martinsburg Depot } 1845 \text { Upon } \\
\text { what meat hath our Ceasar fed } \\
\text { that he hath grown so great }\end{array}$ & 1845 & On Drawing & Martinsburg, VA (WV) & On Drawing & Men at Leisure \\
\hline 328 & W1995.030.394pg34 & $\begin{array}{l}\text { Martinsburg October 2nd, } \\
1859\end{array}$ & $\begin{array}{l}\text { October } 2 \\
1859\end{array}$ & On Drawing & Martinsburg, VA (WV) & On Drawing & $\begin{array}{l}\text { Architecture } \\
\text { (Fences) }\end{array}$ \\
\hline 329 & W1995.030.340 & $\begin{array}{l}\text { Martinsburg, Va. January 11th } \\
1861\end{array}$ & $\begin{array}{c}\text { January } 11 \\
1861\end{array}$ & On Drawing & Martinsburg, VA (WV) & On Drawing & Men at Work \\
\hline 330 & W1995.030.261 & Martinsburg-Va. & 1845 & On Drawing & Martinsburg, VA (WV) & On Drawing & Children \\
\hline 331 & W1995.030.390pg2a & $\begin{array}{l}\text { Max. Woodhull. Washington. } \\
\text { Castor Plant Allgany. } 48 \text { Nov }\end{array}$ & Unknown & $\begin{array}{l}\text { No Date on } \\
\text { Drawing }\end{array}$ & Allegheny County, VA & On Drawing & $\begin{array}{l}\text { African } \\
\text { Americans, } \\
\text { Architecture, } \\
\text { Landscape, } \\
\text { Men, Women }\end{array}$ \\
\hline
\end{tabular}




\begin{tabular}{|c|c|c|c|c|c|c|c|}
\hline Number & Accession Number & Title & Date & $\begin{array}{l}\text { Source of Date } \\
\text { Information }\end{array}$ & Location & $\begin{array}{l}\text { Source of Location } \\
\text { Information }\end{array}$ & Themes \\
\hline 332 & W1995.030.395pg6 & May 5th 1874 & May 5, 1874 & On Drawing & Unknown & No Location on Drawing & Men \\
\hline 333 & W1995.030.390pg13b & McCaffary's 1848. & 1848 & On Drawing & Unknown & No Location on Drawing & $\begin{array}{l}\text { Architecture } \\
\text { (Residential } \\
\text { Buildings) }\end{array}$ \\
\hline 334 & W1995.030.392pg2b & $\begin{array}{l}\text { Mexico City Donkey Country } \\
\text { Donkey Water Carriers at St. } \\
\text { Domingo Watering Streets } \\
\text { Ranchero Peons }\end{array}$ & Unknown & $\begin{array}{l}\text { No Date on } \\
\text { Drawing }\end{array}$ & Mexico City, Mexico & On Drawing & $\begin{array}{l}\text { Animals } \\
\text { (Domestic), } \\
\text { Men at Work }\end{array}$ \\
\hline 335 & W1995.030.393pg25a & $\begin{array}{l}\text { Michaux Slyva Americana } \\
\text { Browne [illegible] trees of } \\
\text { America }\end{array}$ & Unknown & $\begin{array}{l}\text { No Date on } \\
\text { Drawing }\end{array}$ & Unknown & No Location on Drawing & $\begin{array}{l}\text { Animals } \\
\text { (Domestic), } \\
\text { Men at Work, } \\
\text { Transportation } \\
\text { (Carriages), } \\
\text { Women }\end{array}$ \\
\hline 336 & W1995.030.250 & $\begin{array}{l}\text { Milby Stephenson June 2nd } \\
1858 \text { (B \& O Artists } \\
\text { Excursion) }\end{array}$ & June 2,1858 & On Drawing & Unknown & No Location on Drawing & $\begin{array}{l}\text { African } \\
\text { Americans, } \\
\text { Men at Leisure }\end{array}$ \\
\hline 337 & W1995.030.076 & $\begin{array}{l}\text { Military Institute at Lexington } \\
\text { Va., Burned June 12th } 1862\end{array}$ & June 12,1862 & On Drawing & Lexington, VA & On Drawing & $\begin{array}{l}\text { Architecture } \\
\text { (Institutional } \\
\text { Buildings), } \\
\text { Landscape, } \\
\text { Military }\end{array}$ \\
\hline 338 & W1995.030.387pg33b & $\begin{array}{l}\text { Mill pond near Suffolk - April } \\
\text { 3rd } 1856 .\end{array}$ & April 3, 1856 & On Drawing & Suffolk County, VA & On Drawing & $\begin{array}{l}\text { Landscape, } \\
\text { Transportation } \\
\text { (Boats) }\end{array}$ \\
\hline
\end{tabular}




\begin{tabular}{|c|c|c|c|c|c|c|c|}
\hline Number & Accession Number & Title & Date & $\begin{array}{l}\text { Source of Date } \\
\text { Information }\end{array}$ & Location & $\begin{array}{l}\text { Source of Location } \\
\text { Information }\end{array}$ & Themes \\
\hline 339 & W1995.030.157 & $\begin{array}{l}\text { Miss Emma Hardynge the } \\
\text { spiritualist. New Bedford } \\
\text { Mass. May 19, 1859. Entering } \\
\text { New Bedford. May 19, } 1859 \text {. } \\
\text { Providence R.I. May 16, } 1859 \\
\text { New ( ) May 14, } 1859\end{array}$ & $\begin{array}{c}\text { May 14, } \\
\text { 1859/ May } \\
16,1859 / \\
\text { May } 19,1859\end{array}$ & On Drawing & $\begin{array}{l}\text { New Bedford, MA/ } \\
\text { Providence, RI }\end{array}$ & On Drawing & $\begin{array}{l}\text { African } \\
\text { Americans, } \\
\text { Men at Leisure, } \\
\text { Men at Work, } \\
\text { Women at } \\
\text { Work }\end{array}$ \\
\hline 340 & W1995.030.226a & Miss Katie Shaklynt & Unknown & $\begin{array}{l}\text { No Date on } \\
\text { Drawing }\end{array}$ & Unknown & No Location on Drawing & $\begin{array}{l}\text { Architecture } \\
\text { (Residential } \\
\text { Buildings), } \\
\text { Landscape } \\
\text { (Fences) }\end{array}$ \\
\hline 341 & W1995.030.194 & $\begin{array}{l}\text { Missionary being burned by } \\
\text { Indians) }\end{array}$ & $\begin{array}{l}\text { October } 14, \\
1853\end{array}$ & $\begin{array}{c}\text { WVRHC } \\
\text { Description Page }\end{array}$ & Unknown & No Location on Drawing & Men \\
\hline 342 & W1995.030.393pg7a & Montgomery Geb. 7th 1857. & $\begin{array}{c}\text { February } 7 \\
1857\end{array}$ & On Drawing & Montgomery, AL & On Drawing & $\begin{array}{l}\text { Animals } \\
\text { (Domestic), } \\
\text { Transportation } \\
\text { (Carriages) }\end{array}$ \\
\hline 343 & W1995.030.174 & $\begin{array}{l}\text { Montpelier Fishery. Going } \\
\text { out. April 10th } 1856 .\end{array}$ & $\begin{array}{l}\text { April 10, } \\
1856\end{array}$ & On Drawing & Unknown & No Location on Drawing & $\begin{array}{l}\text { Landscape } \\
\text { (Lakes), Men } \\
\text { at Work, } \\
\text { Transportation } \\
\text { (Boats) }\end{array}$ \\
\hline 344 & W1995.030.394pg1 & Monument Mt. Washington & June 25,1859 & On Drawing & Mt. Washington, NH & On Drawing & $\begin{array}{l}\text { Animals } \\
\text { (Domestic), } \\
\text { Landscape } \\
\text { (Mountains), } \\
\text { Men in } \\
\text { Wilderness }\end{array}$ \\
\hline 345 & W1995.030.387pg14 & Moorfield October 1854 & October 1854 & On Drawing & Moorefield, VA (WV) & On Drawing & $\begin{array}{l}\text { Animals } \\
\text { (Domestic) }\end{array}$ \\
\hline
\end{tabular}




\begin{tabular}{|c|c|c|c|c|c|c|c|}
\hline Number & Accession Number & Title & Date & $\begin{array}{l}\text { Source of Date } \\
\text { Information }\end{array}$ & Location & $\begin{array}{l}\text { Source of Location } \\
\text { Information }\end{array}$ & Themes \\
\hline 346 & W1995.030.387pg15 & $\begin{array}{l}\text { Moorfield, October } 1854 \\
\text { Moray Randolph's maid }\end{array}$ & $\begin{array}{l}\text { March 11, } \\
1854\end{array}$ & On Drawing & Clarke County, VA & On Drawing & $\begin{array}{l}\text { Animals } \\
\text { (Domestic) }\end{array}$ \\
\hline 347 & W1995.030.387pg13 & $\begin{array}{l}\text { Morgan Co. September 20th } \\
1846\end{array}$ & $\begin{array}{l}\text { September } \\
20,1846\end{array}$ & On Drawing & $\begin{array}{c}\text { Morgan County, VA } \\
\text { (WV) }\end{array}$ & On Drawing & Landscape \\
\hline 348 & W1995.030.387pg12 & $\begin{array}{l}\text { Morgan Co. Virginia. D.H.S. } \\
1846\end{array}$ & 1846 & On Drawing & $\begin{array}{c}\text { Morgan County, VA } \\
\text { (WV) }\end{array}$ & On Drawing & Landscape \\
\hline 349 & W1995.030.390pg16b & $\begin{array}{l}\text { Morris Hill between Jackson's } \\
\text { River \& Callahan's /5 m. from } \\
\text { Lynchburg- }\end{array}$ & Unknown & $\begin{array}{l}\text { No Date on } \\
\text { Drawing }\end{array}$ & Lynchburg, VA & On Drawing & $\begin{array}{l}\text { African } \\
\text { Americans, } \\
\text { Animals } \\
\text { (Domestic), } \\
\text { Landscape } \\
\text { (Mountains), } \\
\text { Men, } \\
\text { Transportation } \\
\text { (Carriages) }\end{array}$ \\
\hline 350 & W1995.030.394pg10 & $\begin{array}{l}\text { Mother Cary June 9th } \\
\text { Siasconsett. Ginger cakes, } \\
\text { fishing hooks, Porter cheese, } \\
\text { candy biscuit, Codfish } \\
\text { whiskey, \& Tabacco } \\
\text { Nantucket }\end{array}$ & June 9,1859 & On Drawing & Nantucket, MA & On Drawing & $\begin{array}{l}\text { Women at } \\
\text { Leisure }\end{array}$ \\
\hline 351 & W1995.030.172a & Mouth of the Bucket Shaft & May 2, 1887 & $\begin{array}{c}\text { WVRHC } \\
\text { Description Page }\end{array}$ & Unknown & No Location on Drawing & $\begin{array}{l}\text { African } \\
\text { Americans, } \\
\text { Men at Work }\end{array}$ \\
\hline 352 & W1995.030.386pg2a & Mr. Penn breakfasts & Unknown & $\begin{array}{l}\text { No Date on } \\
\text { Drawing }\end{array}$ & $\begin{array}{c}\text { Canaan Valley, VA } \\
\text { (WV) }\end{array}$ & $\begin{array}{c}\text { Strother, "The Virginian } \\
\text { Canaan," Harpers New } \\
\text { Monthly Magazine, } \\
\text { December } 1853,18 .\end{array}$ & $\begin{array}{l}\text { Men at Leisure, } \\
\text { Men in } \\
\text { Wilderness }\end{array}$ \\
\hline
\end{tabular}




\begin{tabular}{|c|c|c|c|c|c|c|c|}
\hline Number & Accession Number & Title & Date & $\begin{array}{l}\text { Source of Date } \\
\text { Information }\end{array}$ & Location & $\begin{array}{l}\text { Source of Location } \\
\text { Information }\end{array}$ & Themes \\
\hline 353 & W1995.030.370 & $\begin{array}{l}\text { Mrs. Bell's residence at } \\
\text { Darristown Montgomery Co. } \\
\text { Maryland August 31st } 1861\end{array}$ & $\begin{array}{c}\text { August 31, } \\
1861\end{array}$ & On Drawing & $\begin{array}{c}\text { Montgomery County, } \\
\text { MD }\end{array}$ & On Drawing & $\begin{array}{l}\text { Architecture } \\
\text { (Residential } \\
\text { Buildings), } \\
\text { Landscape } \\
\text { (Roads) }\end{array}$ \\
\hline 354 & W1995.030.393pg18b & $\begin{array}{l}\text { My name it is Sam 'all Damn } \\
\text { yr eyes }\end{array}$ & $\begin{array}{l}\text { August 16, } \\
\text { 1858/ August } \\
25,1858\end{array}$ & $\begin{array}{c}\text { WVRHC } \\
\text { Description Page }\end{array}$ & Unknown & No Location on Drawing & Men at Leisure \\
\hline 355 & W1995.030.284 & $\begin{array}{l}\text { Nancy and Becky Foster Iron } \\
\text { Mountains East Tennessee } \\
\text { Dec 9th.. } 1856\end{array}$ & $\begin{array}{c}\text { December } 9 \\
1856\end{array}$ & On Drawing & Iron Mountains, TN & On Drawing & Children \\
\hline 356 & W1995.030.393pg21b & Nanny - Jan 8th 1867. & $\begin{array}{l}\text { January } 8 \\
\quad 1867\end{array}$ & On Drawing & Unknown & No Location on Drawing & Children \\
\hline 357 & W1995.030.394pg8 & Nantucket & June 8,1859 & On Drawing & Nantucket, MA & On Drawing & $\begin{array}{l}\text { Men at Work, } \\
\text { Transportation } \\
\text { (Boats) }\end{array}$ \\
\hline 358 & W1995.030.394pg9 & Nantucket/Black Bass & June 8,1859 & On Drawing & Nantucket, MA & On Drawing & $\begin{array}{l}\text { Animals } \\
\text { (Wild), } \\
\text { Transportation } \\
\text { (Boats) }\end{array}$ \\
\hline 359 & W1995.030.075 & $\begin{array}{l}\text { Narrow Passage on the Valley } \\
\text { Turnpike above } \\
\text { WoodstockShenandoah Co. } \\
\text { Va. }\end{array}$ & $1860 \mathrm{~s}$ & $\begin{array}{l}\text { On Drawing } \\
\text { (Exact Date } \\
\text { Unclear) }\end{array}$ & $\begin{array}{c}\text { Shenandoah County, } \\
\text { VA }\end{array}$ & On Drawing & $\begin{array}{l}\text { Landscape } \\
\text { (Mountains), } \\
\text { Men, Military }\end{array}$ \\
\hline
\end{tabular}




\begin{tabular}{|c|c|c|c|c|c|c|c|}
\hline Number & Accession Number & Title & Date & $\begin{array}{l}\text { Source of Date } \\
\text { Information }\end{array}$ & Location & $\begin{array}{l}\text { Source of Location } \\
\text { Information }\end{array}$ & Themes \\
\hline 360 & W1995.030.295 & $\begin{array}{l}\text { Natural Tunnel Scott Co. } \\
\text { Virginia. Looking outward } \\
\text { and down stream }\end{array}$ & $\begin{array}{c}\text { November } \\
1856\end{array}$ & $\begin{array}{c}\text { WVRHC } \\
\text { Description Page }\end{array}$ & Scott County, VA & $\begin{array}{l}\text { See Numbers136, 315, } \\
\text { and } 703\end{array}$ & $\begin{array}{l}\text { Landscape } \\
\text { (Mountains) }\end{array}$ \\
\hline 361 & W1995.030.059 & $\begin{array}{l}\text { Near Cedar Run Mtn. } \\
\text { Culpeper Co., Va. }\end{array}$ & May 25,1863 & $\begin{array}{c}\text { WVRHC } \\
\text { Description Page }\end{array}$ & Culpepper County, VA & On Drawing & $\begin{array}{l}\text { Animals } \\
\text { (Domestic), } \\
\text { Landscape } \\
\text { (Mountains) }\end{array}$ \\
\hline 362 & W1995.030.156 & $\begin{array}{l}\text { Near Edgartown } \\
\text { Vinyard/Squire Flanders Gay } \\
\text { Head Light House/Cape Cod } \\
\text { Pasture }\end{array}$ & 1859 & On Drawing & $\begin{array}{c}\text { Edgarton, NY/ Gay } \\
\text { Head, MA/ Cape Cod, } \\
\text { MA }\end{array}$ & On Drawing & $\begin{array}{l}\text { Animals } \\
\text { (Domestic), } \\
\text { Men, } \\
\text { Transportation } \\
\text { (Carriages), } \\
\text { Women }\end{array}$ \\
\hline 363 & W1995.030.080 & Near Villombrosa, Tuscany & 1842 & On Drawing & Vallombrosa, Italy & On Drawing & $\begin{array}{l}\text { Landscape } \\
\text { (Mountains), } \\
\text { Religious } \\
\text { Shrines }\end{array}$ \\
\hline 364 & W1995.030.051 & Near Woodstock, Va. & April 7, 1862 & On Drawing & Woodstock, VA & On Drawing & $\begin{array}{l}\text { Animals } \\
\text { (Domestic), } \\
\text { Men, Military }\end{array}$ \\
\hline 365 & W1995.030.193 & $\begin{array}{l}\text { New is scace (sic) up here. } \\
\text { Anything encouraging down } \\
\text { your way. }\end{array}$ & Unknown & $\begin{array}{l}\text { No Date on } \\
\text { Drawing }\end{array}$ & Unknown & No Location on Drawing & $\begin{array}{l}\text { Animals } \\
\text { (Domestic), } \\
\text { Men at Work }\end{array}$ \\
\hline 366 & W1995.030.393pg9b & $\begin{array}{l}\text { New Orleans 1857. Sunday } \\
\text { Evening }\end{array}$ & 1857 & On Drawing & New Orleans, LA & On Drawing & Met at Leisure \\
\hline
\end{tabular}




\begin{tabular}{|c|c|c|c|c|c|c|c|}
\hline Number & Accession Number & Title & Date & $\begin{array}{l}\text { Source of Date } \\
\text { Information }\end{array}$ & Location & $\begin{array}{l}\text { Source of Location } \\
\text { Information }\end{array}$ & Themes \\
\hline 367 & W1995.030.001 & New York 1845 & 1845 & On Drawing & New York & On Drawing & $\begin{array}{l}\text { Men at Leisure, } \\
\text { Transportation } \\
\text { (Boats) }\end{array}$ \\
\hline 368 & W1995.030.003 & New York Dec. 12th 1858 & $\begin{array}{c}\text { December 12, } \\
1858\end{array}$ & On Drawing & New York & On Drawing & Men at Leisure \\
\hline 369 & W1995.030.178 & $\begin{array}{l}\text { Nicky Trevathan A Cornish } \\
\text { Man at Gold Hill Mines NC }\end{array}$ & 1856 & On Drawing & $\begin{array}{l}\text { Gold Hill, North } \\
\text { Carolina }\end{array}$ & On Drawing & $\begin{array}{l}\text { Industry, Men } \\
\text { at Work }\end{array}$ \\
\hline 370 & W1995.030.010 & Nine days old & $\begin{array}{l}\text { November 7, } \\
1857\end{array}$ & On Drawing & Unknown & No Location on Drawing & $\begin{array}{l}\text { Animals } \\
\text { (Domestic) }\end{array}$ \\
\hline 371 & W1995.030.256a & Norborne Hall & January 1873 & On Drawing & Martinsburg, VA (WV) & $\begin{array}{l}\text { Cecil Eby, "Porte } \\
\text { Crayon": The Life of } \\
\text { David Hunter Strother, } \\
\text { (Chapel Hill, NC: } \\
\text { University of North } \\
\text { Carolina Press, 1960), } 62 .\end{array}$ & Women \\
\hline 372 & W1995.030.277 & $\begin{array}{l}\text { Norborne. Jan 6th..1877. (En } \\
\text { verso, preliminary pencil } \\
\text { drawings of same woman and } \\
\text { man) }\end{array}$ & $\begin{array}{c}\text { January } 6 \\
1877\end{array}$ & On Drawing & Martinsburg, VA (WV) & $\begin{array}{l}\text { Cecil Eby, "Porte } \\
\text { Crayon": The Life of } \\
\text { David Hunter Strother, } \\
\text { (Chapel Hill, NC: } \\
\text { University of North } \\
\text { Carolina Press, 1960), } 62 .\end{array}$ & Men, Women \\
\hline 373 & W1995.030.278 & Norbourne Hall & $\begin{array}{c}\text { January } 6 \\
1877\end{array}$ & On Drawing & Martinsburg, VA (WV) & $\begin{array}{l}\text { Cecil Eby, "Porte } \\
\text { Crayon": The Life of } \\
\text { David Hunter Strother, } \\
\text { (Chapel Hill, NC: } \\
\text { University of North } \\
\text { Carolina Press, 1960), } 62 .\end{array}$ & Men \\
\hline
\end{tabular}




\begin{tabular}{|c|c|c|c|c|c|c|c|}
\hline Number & Accession Number & Title & Date & $\begin{array}{l}\text { Source of Date } \\
\text { Information }\end{array}$ & Location & $\begin{array}{l}\text { Source of Location } \\
\text { Information }\end{array}$ & Themes \\
\hline 374 & W1995.030.389pg3 & $\begin{array}{l}\text { Norbourne Hall. Jany 6th } \\
1877 .\end{array}$ & $\begin{array}{c}\text { January } 6, \\
1877\end{array}$ & On Drawing & Martinsburg, VA (WV) & $\begin{array}{c}\text { Cecil Eby, "Porte } \\
\text { Crayon": The Life of } \\
\text { David Hunter Strother, } \\
\text { (Chapel Hill, NC: } \\
\text { University of North } \\
\text { Carolina Press, 1960), } 62 .\end{array}$ & Men \\
\hline 375 & W1995.030.348 & $\begin{array}{l}\text { North fork gap. Hardy Co } \\
\text { West Va.. } 15 \text { miles above } \\
\text { Moorfield }\end{array}$ & 1870 & $\begin{array}{c}\text { WVRHC } \\
\text { Description Page }\end{array}$ & $\begin{array}{l}\text { Hardy County, VA } \\
\text { (WV) }\end{array}$ & On Drawing & $\begin{array}{l}\text { Architecture } \\
\text { (Residential } \\
\text { Buildings), } \\
\text { Landscape } \\
\text { (Mountains) }\end{array}$ \\
\hline 376 & W1995.030.395pg7 & $\begin{array}{l}\text { Nov 27th } 1874 \text { Berkeley } \\
\text { Springs Andrew Lee. }\end{array}$ & $\begin{array}{l}\text { November } \\
27,1874\end{array}$ & On Drawing & $\begin{array}{l}\text { Berkeley Springs, VA } \\
\text { (WV) }\end{array}$ & On Drawing & $\begin{array}{l}\text { African } \\
\text { Americans, } \\
\text { Children }\end{array}$ \\
\hline 377 & W1995.030.387pg26a & Nov. 7th 1857. & $\begin{array}{c}\text { November } 7 \\
1857\end{array}$ & On Drawing & Unknown & No Location on Drawing & $\begin{array}{l}\text { Animals } \\
\text { (Domestic) }\end{array}$ \\
\hline 378 & W1995.030.387pg33a & Nov: 2nd 1853. & $\begin{array}{l}\text { November 2, } \\
1853\end{array}$ & On Drawing & Unknown & No Location on Drawing & $\begin{array}{l}\text { Architecture, } \\
\text { Landscape } \\
\text { (Mountains) }\end{array}$ \\
\hline 379 & W1995.030.387pg29 & November 1st 1853 & $\begin{array}{c}\text { November 1, } \\
1853\end{array}$ & On Drawing & Unknown & No Location on Drawing & $\begin{array}{l}\text { Landscape } \\
\text { (Mountains) }\end{array}$ \\
\hline 380 & W1995.030.387pg48 & $\begin{array}{l}\text { November 2nd } 1858 . \\
\text { Baltimore }\end{array}$ & $\begin{array}{l}\text { November 2, } \\
1858\end{array}$ & On Drawing & Baltimore, MD & On Drawing & $\begin{array}{l}\text { Decorative } \\
\text { Arts, Men }\end{array}$ \\
\hline 381 & W1995.030.293 & $\begin{array}{l}\text { Now County Town of Grant } \\
\text { Co. Petersburg } 10 \text { miles above } \\
\text { Moorfield }\end{array}$ & Unknown & $\begin{array}{l}\text { No Date on } \\
\text { Drawing }\end{array}$ & $\begin{array}{l}\text { Grant County, VA } \\
\text { (WV) }\end{array}$ & On Drawing & $\begin{array}{l}\text { Architecture } \\
\text { (Residential } \\
\text { Buildings), } \\
\text { Landscape } \\
\text { (Mountains) }\end{array}$ \\
\hline 382 & W1995.030.388pg16 & Oak/Sugar Maple & Unknown & $\begin{array}{l}\text { No Date on } \\
\text { Drawing }\end{array}$ & Unknown & No Location on Drawing & Landscape \\
\hline
\end{tabular}




\begin{tabular}{|c|c|c|c|c|c|c|c|}
\hline Number & Accession Number & Title & Date & $\begin{array}{l}\text { Source of Date } \\
\text { Information }\end{array}$ & Location & $\begin{array}{l}\text { Source of Location } \\
\text { Information }\end{array}$ & Themes \\
\hline 383 & W1995.030.387pg32 & $\begin{array}{l}\text { Oct. 16th Commenced } 5 \text { 1/2 o } \\
\text { clock A.M. - } 1853\end{array}$ & $\begin{array}{l}\text { October } 16, \\
1853\end{array}$ & On Drawing & Weyer's Cave, VA & See Number 114 & $\begin{array}{l}\text { Landscape } \\
\text { (Caves), Men }\end{array}$ \\
\hline 384 & W1995.030.387pg40 & $\begin{array}{l}\text { Oct. 5th } 1854 \text { - Ridge Road to } \\
\text { Ice Mountain }\end{array}$ & $\begin{array}{l}\text { October } 5 \\
1854\end{array}$ & On Drawing & Unknown & No Location on Drawing & $\begin{array}{l}\text { Landscape } \\
\text { (Mountains), } \\
\text { Transportation } \\
\text { (Carriages) }\end{array}$ \\
\hline 385 & W1995.030.345 & $\begin{array}{l}\text { Oct: } 1854 \text { Moray Randolph's } \\
\text { Hardy Co Va.. }\end{array}$ & October 1854 & On Drawing & $\begin{array}{l}\text { Hardy County, VA } \\
\text { (WV) }\end{array}$ & On Drawing & $\begin{array}{l}\text { African } \\
\text { Americans, } \\
\text { Children }\end{array}$ \\
\hline 386 & W1995.030.387pg30 & Octo: 15 th 1853. & $\begin{array}{l}\text { October } 15 \\
1853\end{array}$ & On Drawing & Weyer's Cave, VA & See Number 718 & $\begin{array}{l}\text { Landscape } \\
\text { (Caves), Men }\end{array}$ \\
\hline 387 & W1995.030.390pg5a & October 1848 & October 1848 & On Drawing & Unknown & No Location on Drawing & Landscape \\
\hline 388 & W1995.030.317 & $\begin{array}{l}\text { October 6th..1854. Potato } \\
\text { Row. Hardy Co. Va.. }\end{array}$ & $\begin{array}{l}\text { October 6, } \\
1854\end{array}$ & On Drawing & $\begin{array}{l}\text { Hardy County, VA } \\
\text { (WV) }\end{array}$ & On Drawing & $\begin{array}{l}\text { Architecture } \\
\text { (Residential } \\
\text { Buildings), } \\
\text { Landscape } \\
\text { (Mountains), } \\
\text { Men }\end{array}$ \\
\hline 389 & W1995.030.390pg4a & $\begin{array}{l}\text { Officers -en Route (various } \\
\text { caricatures) }\end{array}$ & Unknown & $\begin{array}{l}\text { No Date on } \\
\text { Drawing }\end{array}$ & Unknown & No Location on Drawing & $\begin{array}{l}\text { Landscape, } \\
\text { Men at Leisure }\end{array}$ \\
\hline 390 & W1995.030.017 & Ohio River & April 1857 & On Drawing & Unknown & No Location on Drawing & $\begin{array}{l}\text { Landscape } \\
\text { (Rivers), Men } \\
\text { at Work, } \\
\text { Transportation } \\
\text { (Boats) }\end{array}$ \\
\hline 391 & W1995.030.019 & Old Fort Below Fairhaven & Unknown & $\begin{array}{l}\text { Date on Drawing } \\
\text { Unclear }\end{array}$ & Unknown & No Location on Drawing & $\begin{array}{l}\text { Architecture, } \\
\text { Landscape, } \\
\text { Men at Leisure, } \\
\text { Military }\end{array}$ \\
\hline
\end{tabular}




\begin{tabular}{|c|c|c|c|c|c|c|c|}
\hline Number & Accession Number & Title & Date & $\begin{array}{l}\text { Source of Date } \\
\text { Information }\end{array}$ & Location & $\begin{array}{l}\text { Source of Location } \\
\text { Information }\end{array}$ & Themes \\
\hline 392 & W1995.030.146 & $\begin{array}{l}\text { Old Fort Frederick from hill } \\
\text { Commanding it \& looking } \\
\text { Southward }\end{array}$ & $\begin{array}{c}\text { November } 4, \\
1877\end{array}$ & On Drawing & Frederick, MD & On Drawing & $\begin{array}{l}\text { Architecture } \\
\text { (Residential } \\
\text { Buildings), } \\
\text { Landscape } \\
\text { (Mountains), } \\
\text { Military }\end{array}$ \\
\hline 393 & W1995.030.371 & $\begin{array}{l}\text { Old Fort Frederick Looking } \\
\text { toward the Potomac River }\end{array}$ & $\begin{array}{c}\text { November } \\
1877\end{array}$ & On Drawing & Frederick, MD & On Drawing & $\begin{array}{l}\text { Architecture } \\
\text { (Residential } \\
\text { Buildings), } \\
\text { Landscape, } \\
\text { Military }\end{array}$ \\
\hline 394 & W1995.030.124 & $\begin{array}{l}\text { Old Spanish Residence New } \\
\text { Orleans }\end{array}$ & $\begin{array}{l}\text { February } 29 \\
\quad \text { Year } \\
\text { Unknown) }\end{array}$ & On Drawing & New Orleans, LA & On Drawing & $\begin{array}{l}\text { Architecture } \\
\text { (Residential } \\
\text { Buildings) }\end{array}$ \\
\hline 395 & W1995.030.395pg1 & $\begin{array}{l}\text { Old Tom, Berkeley Springs, } \\
\text { West Va Nov. 16th 1864; A } \\
\text { Reminence of the War, } \\
\text { Kanawha } 1872 \text { May 11th; } \\
\text { Kanawha May 11th 1872; } \\
\text { Kanawha May 13th 1872; } \\
\text { June 26th 1867. Berkeley } \\
\text { Springs West Va. }\end{array}$ & $\begin{array}{c}\text { November } \\
\text { 16, 1864/ } \\
\text { June 26, } \\
\text { 1867/ May } \\
\text { 11, 1872/ } \\
\text { May 13, 1872 }\end{array}$ & On Drawing & $\begin{array}{l}\text { Berkeley Springs, VA } \\
\text { (WV)/ Kanawha, WV }\end{array}$ & On Drawing & $\begin{array}{l}\text { Animals } \\
\text { (Domestic), } \\
\text { Children, Men }\end{array}$ \\
\hline 396 & W1995.030.129 & $\begin{array}{l}\text { On the Bayou Tesche- La- } \\
\text { March 10th } 1857\end{array}$ & $\begin{array}{l}\text { March 10, } \\
\quad 1857\end{array}$ & On Drawing & $\begin{array}{l}\text { Bayou Teche, St. } \\
\text { Mary's Parish, LA }\end{array}$ & On Drawing & $\begin{array}{l}\text { Architecture } \\
\text { (Residential } \\
\text { Buildings), } \\
\text { Landscape } \\
\text { (Lakes) }\end{array}$ \\
\hline
\end{tabular}




\begin{tabular}{|c|c|c|c|c|c|c|c|}
\hline Number & Accession Number & Title & Date & $\begin{array}{l}\text { Source of Date } \\
\text { Information }\end{array}$ & Location & $\begin{array}{l}\text { Source of Location } \\
\text { Information }\end{array}$ & Themes \\
\hline 397 & W1995.030.341 & $\begin{array}{l}\text { On the Flat at Elk River Dam } \\
\text { Kanawha May 10th..1872 }\end{array}$ & May 10,1875 & On Drawing & $\begin{array}{c}\text { Kanawha County, VA } \\
\text { (WV) }\end{array}$ & On Drawing & $\begin{array}{l}\text { Men at Work, } \\
\text { Transportation } \\
\text { (Boats) }\end{array}$ \\
\hline 398 & W1995.030.387pg11 & $\begin{array}{l}\text { On the road above Meyers } \\
\text { Berkeley Springs September } \\
\text { 21st } 1845\end{array}$ & $\begin{array}{l}\text { September } \\
21,1845\end{array}$ & On Drawing & $\begin{array}{l}\text { Berkeley Springs, VA } \\
\text { (WV) }\end{array}$ & On Drawing & Landscape \\
\hline 399 & W1995.030.395pg10 & $\begin{array}{l}\text { On The Steamer Clara Scott } \\
\text { Kanawha River Four Years } \\
\text { Old }\end{array}$ & Unknown & $\begin{array}{l}\text { No Date on } \\
\text { Drawing }\end{array}$ & $\begin{array}{c}\text { Kanawha County, VA } \\
\text { (WV) }\end{array}$ & On Drawing & Children \\
\hline 400 & W1995.030.152a & $\begin{array}{l}\text { One of the Aztec Children } \\
\text { City of Mexico } 1880 .\end{array}$ & 1880 & On Drawing & Mexico City, Mexico & On Drawing & Men \\
\hline 401 & W1995.030.043 & $\begin{array}{l}\text { Only near Onancock- Former } \\
\text { residence of Henry A. Wise }\end{array}$ & $\begin{array}{l}\text { April 20, } \\
1887\end{array}$ & On Drawing & Accomack County, VA & On Drawing & $\begin{array}{l}\text { Architecture } \\
\text { (Residential } \\
\text { Buildings), } \\
\text { Men at Work }\end{array}$ \\
\hline 402 & W1995.030.388pg1 & Orfeo & 1846 & $\begin{array}{c}\text { WVRHC } \\
\text { Description Page }\end{array}$ & Unknown & No Location on Drawing & $\begin{array}{l}\text { Animals } \\
\text { (Domestic), } \\
\text { Men, Women }\end{array}$ \\
\hline 403 & W1995.030.387pg39 & $\begin{array}{l}\text { Our Cow Norbourne April } \\
\text { 16th } 1858 .\end{array}$ & $\begin{array}{l}\text { April 16, } \\
1858\end{array}$ & On Drawing & Martinsburg, VA (WV) & $\begin{array}{c}\text { Cecil Eby, "Porte } \\
\text { Crayon": The Life of } \\
\text { David Hunter Strother, } \\
\text { (Chapel Hill, NC: } \\
\text { University of North } \\
\text { Carolina Press, 1960), } 62 .\end{array}$ & $\begin{array}{l}\text { Animals } \\
\text { (Domestic), } \\
\text { Architecture } \\
\text { (Residential } \\
\text { Buildings) }\end{array}$ \\
\hline
\end{tabular}




\begin{tabular}{|c|c|c|c|c|c|c|c|}
\hline Number & Accession Number & Title & Date & $\begin{array}{l}\text { Source of Date } \\
\text { Information }\end{array}$ & Location & $\begin{array}{l}\text { Source of Location } \\
\text { Information }\end{array}$ & Themes \\
\hline 404 & W1995.030.006 & Park Forest & April 2, 1859 & On Drawing & $\begin{array}{l}\text { Charles Town, VA } \\
\text { (WV) }\end{array}$ & $\begin{array}{c}\text { Cecil Eby, "Porte } \\
\text { Crayon": The Life of } \\
\text { David Hunter Strother, } \\
\text { (Chapel Hill, NC: } \\
\text { University of North } \\
\text { Carolina Press, 1960), } 4 .\end{array}$ & $\begin{array}{l}\text { Animals } \\
\text { (Domestic) }\end{array}$ \\
\hline 405 & W1995.030.020 & Park Forest & $\begin{array}{l}\text { March 31, } \\
\quad 1859\end{array}$ & On Drawing & $\begin{array}{l}\text { Charles Town, VA } \\
\text { (WV) }\end{array}$ & $\begin{array}{l}\text { Cecil Eby, "Porte } \\
\text { Crayon": The Life of } \\
\text { David Hunter Strother, } \\
\text { (Chapel Hill, NC: } \\
\text { University of North } \\
\text { Carolina Press, 1960), } 4 .\end{array}$ & $\begin{array}{l}\text { Animals } \\
\text { (Domestic), } \\
\text { Architecture } \\
\text { (Residential } \\
\text { Buildings) }\end{array}$ \\
\hline 406 & W1995.030.383 & Passing the Laurel & July 22,1858 & $\begin{array}{c}\text { WVRHC } \\
\text { Description Page }\end{array}$ & $\begin{array}{c}\text { Canaan Valley, VA } \\
\text { (WV) }\end{array}$ & $\begin{array}{c}\text { Strother, "The Virginian } \\
\text { Canaan," Harpers New } \\
\text { Monthly Magazine, } \\
\text { December } 1853,26 .\end{array}$ & $\begin{array}{l}\text { Men in } \\
\text { Wilderness }\end{array}$ \\
\hline 407 & W1995.030.022 & $\begin{array}{l}\text { Pendleton Co. West Va. } \\
\text { Pinnacles at Adam Carr's }\end{array}$ & Unknown & $\begin{array}{l}\text { No Date on } \\
\text { Drawing }\end{array}$ & $\begin{array}{l}\text { Pendleton County, VA } \\
\text { (WV) }\end{array}$ & On Drawing & $\begin{array}{l}\text { Landscape } \\
\text { (Mountains) }\end{array}$ \\
\hline 408 & W1995.030.388pg9a & Pentolaccio & 1871 & $\begin{array}{c}\text { WVRHC } \\
\text { Description Page }\end{array}$ & Unknown & No Location on Drawing & Children \\
\hline 409 & W1995.030.077 & Pentolaccio- Smash Egg & 1852 & $\begin{array}{c}\text { WVRHC } \\
\text { Description Page }\end{array}$ & Unknown & No Location on Drawing & Children \\
\hline 410 & W1995.030.315 & $\begin{array}{l}\text { Petersburg Gap-Southern gate } \\
\text { to the South branch valley }\end{array}$ & $\begin{array}{l}\text { December 2, } \\
1857\end{array}$ & $\begin{array}{c}\text { WVRHC } \\
\text { Description Page }\end{array}$ & Petersburg, VA (WV) & On Drawing & $\begin{array}{l}\text { Landscape } \\
\text { (Mountains), } \\
\text { Landscape } \\
\text { (Rivers) }\end{array}$ \\
\hline
\end{tabular}




\begin{tabular}{|c|c|c|c|c|c|c|c|}
\hline Number & Accession Number & Title & Date & $\begin{array}{l}\text { Source of Date } \\
\text { Information }\end{array}$ & Location & $\begin{array}{l}\text { Source of Location } \\
\text { Information }\end{array}$ & Themes \\
\hline 411 & W1995.030.387pg47 & $\begin{array}{l}\text { Philip E. Thomas. Baltimore, } \\
\text { October 25th } 1858\end{array}$ & $\begin{array}{l}\text { October } 25, \\
1858\end{array}$ & On Drawing & Baltimore, MD & On Drawing & Men \\
\hline 412 & W1995.030.221a & Pig catching & 1845 & $\begin{array}{c}\text { WVRHC } \\
\text { Description Page }\end{array}$ & Unknown & No Location on Drawing & $\begin{array}{l}\text { African } \\
\text { Americans, } \\
\text { Animals } \\
\text { (Domestic), } \\
\text { Men at Work }\end{array}$ \\
\hline 413 & W1995.030.161a & $\begin{array}{l}\text { Planked Shad Indian Head } \\
\text { Fishery - May } 5,1859 .\end{array}$ & May 5, 1859 & On Drawing & Indian Head, MD & $\begin{array}{c}\text { Jessie Poesch, "Eight } \\
\text { Drawings by David } \\
\text { Hunter Strother in the } \\
\text { Walters Collection,” The } \\
\text { Journal of the Walters Art } \\
\text { Gallery 52/53 } \\
\text { (1994/1995): } 75 .\end{array}$ & Animals (Wild) \\
\hline 414 & W1995.030.144 & $\begin{array}{l}\text { Plantation Residences on the } \\
\text { Mississippi Above New } \\
\text { Orleans Louisiana }\end{array}$ & May 1,1887 & $\begin{array}{c}\text { WVRHC } \\
\text { Description Page }\end{array}$ & New Orleans, LA & $\begin{array}{c}\text { WVRHC Description } \\
\text { Page }\end{array}$ & \\
\hline 415 & W1995.030.393pg13a & Planting Corn - May 4th 1858 & May 4, 1858 & On Drawing & Unknown & No Location on Drawing & $\begin{array}{l}\text { Animals } \\
\text { (Domestic), } \\
\text { Children, Men } \\
\text { at Work }\end{array}$ \\
\hline 416 & W1995.030.301 & Point of Jamestown Island & $\begin{array}{l}\text { October } 14 \\
1853\end{array}$ & $\begin{array}{c}\text { WVRHC } \\
\text { Description Page }\end{array}$ & Jamestown, VA & On Drawing & $\begin{array}{l}\text { Landscape } \\
\text { (River), Men at } \\
\text { Work, } \\
\text { Transportation } \\
\text { (Boats) }\end{array}$ \\
\hline 417 & W1995.030.038 & Pompei after Raphael & $\begin{array}{l}\text { August } 10 \\
1873\end{array}$ & $\begin{array}{c}\text { WVRHC } \\
\text { Description Page }\end{array}$ & Unknown & No Location on Drawing & $\begin{array}{l}\text { Copies of } \\
\text { European } \\
\text { Artwork }\end{array}$ \\
\hline
\end{tabular}




\begin{tabular}{|c|c|c|c|c|c|c|c|}
\hline Number & Accession Number & Title & Date & $\begin{array}{l}\text { Source of Date } \\
\text { Information }\end{array}$ & Location & $\begin{array}{l}\text { Source of Location } \\
\text { Information }\end{array}$ & Themes \\
\hline 418 & W1995.030.387pg17a & Pompel's Story Dec. 2. 1858 & $\begin{array}{l}\text { December 2, } \\
1858\end{array}$ & On Drawing & Unknown & No Location on Drawing & Men \\
\hline 419 & W1995.030.097 & $\begin{array}{l}\text { Porta San Giovanni Roma } \\
\text { (See Remarks) }\end{array}$ & March 1842 & On Drawing & Rome, Italy & On Drawing & $\begin{array}{l}\text { Architecture } \\
\text { (Towns), } \\
\text { Landscape } \\
\text { (Mountains) }\end{array}$ \\
\hline 420 & W2002.088.001 & $\begin{array}{l}\text { Portrait of a Child (Clara } \\
\text { Hunter) }\end{array}$ & Unknown & $\begin{array}{l}\text { No Date on } \\
\text { Painting }\end{array}$ & Unknown & No Location on Painting & Children \\
\hline 421 & W1995.030.392pg10 & Powelton. May 2nd..1887 & May 2,1887 & On Drawing & Accomack County, VA & $\begin{array}{c}\text { Cecil Eby, "Porte } \\
\text { Crayon": The Life of } \\
\text { David Hunter Strother, } \\
\text { (Chapel Hill, NC: } \\
\text { University of North } \\
\text { Carolina Press, 1960), } \\
204 .\end{array}$ & $\begin{array}{l}\text { Women at } \\
\text { Leisure }\end{array}$ \\
\hline 422 & W1995.030.085 & $\begin{array}{l}\text { Raphael's House Villa } \\
\text { Borghese Rome }\end{array}$ & March 1842 & On Drawing & Rome, Italy & On Drawing & $\begin{array}{l}\text { Architecture } \\
\text { (Residential } \\
\text { Buildings) }\end{array}$ \\
\hline 423 & W1995.030.381 & $\begin{array}{l}\text { Rapt Artist (self portrait } \\
\text { Virginia Canaan 1852) }\end{array}$ & 1852 & $\begin{array}{c}\text { WVRHC } \\
\text { Description Page }\end{array}$ & $\begin{array}{c}\text { Canaan Valley, VA } \\
\text { (WV) }\end{array}$ & $\begin{array}{l}\text { WVRHC Description } \\
\text { Page }\end{array}$ & $\begin{array}{l}\text { Landscape } \\
\text { (Waterfalls), } \\
\text { Men in } \\
\text { Wilderness }\end{array}$ \\
\hline 424 & W1995.030.049 & $\begin{array}{l}\text { Rear Guard of the Indiana } \\
\text { Volunteers, Damstown }\end{array}$ & $\begin{array}{l}\text { September } \\
16,1861\end{array}$ & On Drawing & $\begin{array}{c}\text { Montgomery County, } \\
\text { MD }\end{array}$ & See Number 353 & Men, Military \\
\hline
\end{tabular}




\begin{tabular}{|c|c|c|c|c|c|c|c|}
\hline Number & Accession Number & Title & Date & $\begin{array}{l}\text { Source of Date } \\
\text { Information }\end{array}$ & Location & $\begin{array}{l}\text { Source of Location } \\
\text { Information }\end{array}$ & Themes \\
\hline 425 & W1995.030.057 & $\begin{array}{l}\text { Remains of the Railway } \\
\text { Bridge at Martinsburg, Va. }\end{array}$ & $1860 \mathrm{~s}$ & $\begin{array}{l}\text { On Drawing } \\
\text { (Exact Date } \\
\text { Unclear) }\end{array}$ & Martinsburg, VA (WV) & On Drawing & $\begin{array}{l}\text { Architecture } \\
\text { (Bridges), } \\
\text { Children, Men } \\
\text { at Leisure }\end{array}$ \\
\hline 426 & W1995.030.175 & Repose of Fishermen & $\begin{array}{l}\text { April } 18 \\
\text { (Year } \\
\text { Unknown) }\end{array}$ & On Drawing & Unknown & No Location on Drawing & $\begin{array}{l}\text { African } \\
\text { Americans, } \\
\text { Men at Leisure }\end{array}$ \\
\hline 427 & W1995.030.393pg21a & Repose. & Unknown & $\begin{array}{l}\text { No Date on } \\
\text { Drawing }\end{array}$ & Unknown & No Location on Drawing & Men at Leisure \\
\hline 428 & W1995.030.393pg24a & $\begin{array}{l}\text { Resurgam Aunt Sally - May 3, } \\
1858\end{array}$ & May 3,1858 & On Drawing & Unknown & No Location on Drawing & $\begin{array}{l}\text { African } \\
\text { Americans, } \\
\text { Women at } \\
\text { Leisure }\end{array}$ \\
\hline 429 & W1995.030.393pg16b & Riley’s Davy Dec 2nd 1857. & $\begin{array}{l}\text { December } 2, \\
1857\end{array}$ & On Drawing & Unknown & No Location on Drawing & $\begin{array}{l}\text { African } \\
\text { Americans, } \\
\text { Men }\end{array}$ \\
\hline 430 & W1995.030.391pg15 & Ringer Maarinsburg 1844. & 1844 & On Drawing & Martinsburg, VA (WV) & On Drawing & Children \\
\hline 431 & W1995.030.304 & $\begin{array}{l}\text { Rockbridge Alum Oct. 29th.. } \\
\text { (en verso--mountain home) }\end{array}$ & $\begin{array}{l}\text { October } 29 \\
\quad \text { (Year } \\
\text { Unknown) }\end{array}$ & On Drawing & $\begin{array}{l}\text { Rockbridge County, } \\
\text { VA }\end{array}$ & $\frac{\text { http://exhibits.hsl.virginia. }}{\text { edu/springs/rockbridge/ }}$ & $\begin{array}{l}\text { Architecture } \\
\text { (Institutional } \\
\text { Buildings), } \\
\text { Landscape } \\
\text { (Mountains) }\end{array}$ \\
\hline 432 & W1995.030.350 & $\begin{array}{l}\text { Ruins of the Old Academy at } \\
\text { Martinsburg January } \\
\text { 18th..1876 }\end{array}$ & $\begin{array}{l}\text { January } 18 \\
\quad 1876\end{array}$ & On Drawing & Martinsburg, VA (WV) & On Drawing & $\begin{array}{l}\text { Architecture } \\
\text { (Industry), } \\
\text { Landscape }\end{array}$ \\
\hline 433 & W1995.030.033 & $\begin{array}{l}\text { Sally Port of Fort Frederick } \\
\text { Looking Outward }\end{array}$ & $\begin{array}{l}\text { November } 1, \\
1877\end{array}$ & On Drawing & Frederick, MD & On Drawing & $\begin{array}{l}\text { Landscape } \\
\text { (Fences), } \\
\text { Military }\end{array}$ \\
\hline
\end{tabular}




\begin{tabular}{|c|c|c|c|c|c|c|c|}
\hline Number & Accession Number & Title & Date & $\begin{array}{l}\text { Source of Date } \\
\text { Information }\end{array}$ & Location & $\begin{array}{l}\text { Source of Location } \\
\text { Information }\end{array}$ & Themes \\
\hline 434 & W1995.030.389pg6 & $\begin{array}{l}\text { Salt Works Destroyed. Drawn } \\
\text { Oct: } 28 \text { th } 1865 .\end{array}$ & $\begin{array}{l}\text { October 28, } \\
1865\end{array}$ & On Drawing & Unknown & No Location on Drawing & Industry \\
\hline 435 & W1995.030.182 & Salt Works Ike & $\begin{array}{l}\text { November } \\
18,1856\end{array}$ & On Drawing & Unknown & No Location on Drawing & $\begin{array}{l}\text { African } \\
\text { Americans, } \\
\text { Industry, Men } \\
\text { at Work }\end{array}$ \\
\hline 436 & W1995.030.013 & Sam & $\begin{array}{c}\text { December } \\
1857\end{array}$ & On Drawing & Unknown & No Location on Drawing & Men at Work \\
\hline 437 & W1995.030.199 & Sausage Raw Cincenati [sic] & May 4,1858 & $\begin{array}{c}\text { WVRHC } \\
\text { Description Page }\end{array}$ & Cincinnati, $\mathrm{OH}$ & On Drawing & $\begin{array}{l}\text { Architecture } \\
\text { (Towns), Men } \\
\text { at Work, } \\
\text { Transportation } \\
\text { (Boats) }\end{array}$ \\
\hline 438 & W1995.030.034 & $\begin{array}{l}\text { Scene on the Potomac } \\
\text { Opposite Fort Frederick }\end{array}$ & $\begin{array}{l}\text { November 2, } \\
1877\end{array}$ & On Drawing & Frederick, MD & On Drawing & $\begin{array}{l}\text { Landscape } \\
\text { (Mountains), } \\
\text { Landscape } \\
\text { (River) }\end{array}$ \\
\hline 439 & W1995.030.159 & $\begin{array}{l}\text { School at Gray Head. June } \\
1859 \text { Schoolhouse at } \\
\text { childmark vineyard }\end{array}$ & June 1859 & On Drawing & Gay Head, MA & See Number 223 & $\begin{array}{l}\text { Architecture } \\
\text { (Institutional } \\
\text { Buildings), } \\
\text { Children, } \\
\text { Women at } \\
\text { Work }\end{array}$ \\
\hline 440 & W1995.030.015 & Schooner Algonquin & 1859 & On Drawing & Unknown & No Location on Drawing & $\begin{array}{l}\text { Animals } \\
\text { (Wild), } \\
\text { Landscape } \\
\text { (Rivers), Men } \\
\text { at Leisure, } \\
\text { Transportation } \\
\text { (Boats) }\end{array}$ \\
\hline
\end{tabular}




\begin{tabular}{|c|c|c|c|c|c|c|c|}
\hline Number & Accession Number & Title & Date & $\begin{array}{l}\text { Source of Date } \\
\text { Information }\end{array}$ & Location & $\begin{array}{l}\text { Source of Location } \\
\text { Information }\end{array}$ & Themes \\
\hline 441 & W1995.030.012 & September 13,1858 & $\begin{array}{l}\text { September } \\
13,1858\end{array}$ & On Drawing & Unknown & No Location on Drawing & Men \\
\hline 442 & W1995.030.394pg24a & $\begin{array}{l}\text { Sharp's Carbine used by John } \\
\text { Brown at Harper's Ferry, Oct. } \\
\text { 16, 17, 18th, 1859. Sketched } \\
\text { in George Murphy's room at } \\
\text { Martinsburg, Oct. 19th. }\end{array}$ & $\begin{array}{l}\text { October } 19 \\
\quad 1859\end{array}$ & On Drawing & Martinsburg, VA (WV) & On Drawing & $\begin{array}{l}\text { Harper's Ferry } \\
\text { Raid Trial }\end{array}$ \\
\hline 443 & W1995.030.393pg3 & $\begin{array}{l}\text { Shell mound Station Near } \\
\text { Nick a jack Cave }\end{array}$ & Unknown & $\begin{array}{l}\text { No Date on } \\
\text { Drawing }\end{array}$ & Chattanooga, TN & See Number 201 & $\begin{array}{l}\text { African } \\
\text { Americans, } \\
\text { Animals } \\
\text { (Domestic), } \\
\text { Architecture, } \\
\text { Landscape } \\
\text { (Rivers), Men } \\
\text { at Work, } \\
\text { Transportation } \\
\text { (Boats) }\end{array}$ \\
\hline 444 & W1995.030.393pg4a & $\begin{array}{l}\text { Shell mound staton } \\
\text { Chattannoga \& Nashville R.R. }\end{array}$ & Unknown & $\begin{array}{l}\text { No Date on } \\
\text { Drawing }\end{array}$ & Chattanooga, TN & On Drawing & $\begin{array}{l}\text { African } \\
\text { Americans, } \\
\text { Women a } \\
\text { Work }\end{array}$ \\
\hline 445 & W1995.030.394pg24b & $\begin{array}{l}\text { Shields Green, on of John } \\
\text { Brown's Negroes going to } \\
\text { Execution }\end{array}$ & Unknown & $\begin{array}{l}\text { No Date on } \\
\text { Drawing }\end{array}$ & Unknown & No Location on Drawing & $\begin{array}{l}\text { African } \\
\text { Americans, } \\
\text { Harper's Ferry } \\
\text { Raid Trial, } \\
\text { Men }\end{array}$ \\
\hline 446 & W1995.030.318 & $\begin{array}{l}\text { Siesta-at Carr's Sketching.-the } \\
\text { Falls of the Blackwater. } \\
\text { Sunday-July 2nd..1854 }\end{array}$ & July 2,1854 & On Drawing & $\begin{array}{c}\text { Canaan Valley, VA } \\
\text { (WV) }\end{array}$ & On Drawing & $\begin{array}{l}\text { Men at Leisure, } \\
\text { Men in } \\
\text { Wilderness }\end{array}$ \\
\hline 447 & W1995.030.362 & $\begin{array}{l}\text { Simeon Hederick Sept. } \\
\text { 30th..1860 Randolph Co. } \\
\text { Virginia }\end{array}$ & $\begin{array}{l}\text { September } \\
30,1860\end{array}$ & On Drawing & $\begin{array}{l}\text { Randolph County, VA } \\
\text { (WV) }\end{array}$ & On Drawing & $\begin{array}{l}\text { Men at Work, } \\
\text { Men in } \\
\text { Wilderness }\end{array}$ \\
\hline
\end{tabular}




\begin{tabular}{|c|c|c|c|c|c|c|c|}
\hline Number & Accession Number & Title & Date & $\begin{array}{l}\text { Source of Date } \\
\text { Information }\end{array}$ & Location & $\begin{array}{l}\text { Source of Location } \\
\text { Information }\end{array}$ & Themes \\
\hline 448 & W1995.030.387pg21 & $\begin{array}{l}\text { Sir John's April 19th } 1859 \\
\text { Bath April 26th } 1859\end{array}$ & $1858 / 1859$ & On Drawing & Clarke County, VA & On Drawing & $\begin{array}{l}\text { Animals } \\
\text { (Domestic), } \\
\text { Men at Leisure }\end{array}$ \\
\hline 449 & W1995.030.387pg17b & $\begin{array}{l}\text { Sir John's Nov: 24th } 1858 . \\
\text { Norborne. Dec. } 1858\end{array}$ & $\begin{array}{c}\text { November } \\
24,1858 / \\
\text { December } \\
1858\end{array}$ & On Drawing & Martinsburg, VA (WV) & $\begin{array}{c}\text { Cecil Eby, "Porte } \\
\text { Crayon": The Life of } \\
\text { David Hunter Strother, } \\
\text { (Chapel Hill, NC: } \\
\text { University of North } \\
\text { Carolina Press, 1960), } 62 .\end{array}$ & Men \\
\hline 450 & W1995.030.387pg1 & Sir John’s Road May, 1851. & Unknown & $\begin{array}{l}\text { No Date on } \\
\text { Drawing }\end{array}$ & $\begin{array}{l}\text { Berkeley Springs, VA } \\
\text { (WV) }\end{array}$ & On Drawing & $\begin{array}{l}\text { Architecture, } \\
\text { Landscape } \\
\text { (Fences), } \\
\text { Transportation } \\
\text { (Carriages) }\end{array}$ \\
\hline 451 & W1995.030.393pg13b & Snuff & Unknown & $\begin{array}{l}\text { No Date on } \\
\text { Drawing }\end{array}$ & Unknown & No Location on Drawing & $\begin{array}{l}\text { Women at } \\
\text { Leisure }\end{array}$ \\
\hline 452 & W1995.030.387pg7 & Soap locks Baltimore-1845 & 1845 & On Drawing & Baltimore, MD & On Drawing & $\begin{array}{l}\text { Children, Men } \\
\text { at Leisure, } \\
\text { Transportation } \\
\text { (Boats) }\end{array}$ \\
\hline 453 & W1995.030.387pg16a & $\begin{array}{l}\text { Soldier's Retreat. Clarke Co } \\
\text { Va. March 11th } 1859\end{array}$ & $\begin{array}{l}\text { March 11, } \\
\quad 1859\end{array}$ & $\begin{array}{c}\text { WVRHC } \\
\text { Description Page }\end{array}$ & Clarke County, VA & On Drawing & $\begin{array}{l}\text { Men at Work, } \\
\text { Transportation } \\
\text { (Carriages) }\end{array}$ \\
\hline 454 & W1995.030.288 & $\begin{array}{l}\text { Soldier's Retreat. Clarke Co. } \\
\text { Va. }\end{array}$ & $\begin{array}{l}\text { March 12, } \\
\quad 1859\end{array}$ & On Drawing & Clarke County, VA & On Drawing & Animals \\
\hline
\end{tabular}




\begin{tabular}{|c|c|c|c|c|c|c|c|}
\hline Number & Accession Number & Title & Date & $\begin{array}{l}\text { Source of Date } \\
\text { Information }\end{array}$ & Location & $\begin{array}{l}\text { Source of Location } \\
\text { Information }\end{array}$ & Themes \\
\hline 455 & W1995.030.386pg2b & Something gets in Jone's eye & Unknown & $\begin{array}{l}\text { No Date on } \\
\text { Drawing }\end{array}$ & $\begin{array}{c}\text { Canaan Valley, VA } \\
\text { (WV) }\end{array}$ & $\begin{array}{c}\text { Strother, "The Virginian } \\
\text { Canaan," Harpers New } \\
\text { Monthly Magazine, } \\
\text { December 1853, } 28 .\end{array}$ & $\begin{array}{l}\text { Men in } \\
\text { Wilderness }\end{array}$ \\
\hline 456 & W1995.030.090 & Sotto Ponte Vecchio Firenze & 1842 & On Drawing & Florence, Italy & On Drawing & $\begin{array}{l}\text { Architecture } \\
\text { (Bridges), } \\
\text { Architecture } \\
\text { (Towns), } \\
\text { Landscape } \\
\text { (Rivers), Men } \\
\text { at Work, } \\
\text { Transportation } \\
\text { (Boats) }\end{array}$ \\
\hline 457 & W1995.030.244a & Sources of Political Power & $\begin{array}{l}\text { March 29, } \\
\quad 1857\end{array}$ & $\begin{array}{c}\text { WVRHC } \\
\text { Description Page }\end{array}$ & Unknown & No Location on Drawing & Men \\
\hline 458 & W1995.030.333 & $\begin{array}{l}\text { South Peak of Otter Nov } \\
\text { 3rd..Thursday }\end{array}$ & $\begin{array}{c}\text { November 3, } \\
1853\end{array}$ & See Number 166 & Botetourt County, VA & See Number 166 & $\begin{array}{l}\text { Landscape } \\
\text { (Mountains), } \\
\text { Men in } \\
\text { Wilderness }\end{array}$ \\
\hline 459 & W1995.030.329 & $\begin{array}{l}\text { Stack Rocks on the Big Plains } \\
\text { of Allegheny. Sept. } \\
\text { 25th..1860. West Va.. }\end{array}$ & $\begin{array}{l}\text { September } \\
25,1860\end{array}$ & On Drawing & West Virginia & On Drawing & $\begin{array}{l}\text { Animals } \\
\text { (Domestic), } \\
\text { Landscape }\end{array}$ \\
\hline 460 & W1995.030.066 & Staff Office Rallying & Unknown & $\begin{array}{l}\text { No Date on } \\
\text { Drawing }\end{array}$ & Unknown & No Location on Drawing & $\begin{array}{l}\text { Men at Leisure, } \\
\text { Military }\end{array}$ \\
\hline 461 & W1995.030.331 & $\begin{array}{l}\text { Steamboat Building in } \\
\text { Wheeling }\end{array}$ & $\begin{array}{l}\text { November } \\
29,1873\end{array}$ & $\begin{array}{c}\text { WVRHC } \\
\text { Description Page }\end{array}$ & Wheeling, VA (WV) & On Drawing & $\begin{array}{l}\text { Architecture } \\
\text { (Towns), } \\
\text { Landscape } \\
\text { (Mountains), } \\
\text { Landscape } \\
\text { (Rivers), } \\
\text { Transportation } \\
\text { (Boats) }\end{array}$ \\
\hline
\end{tabular}




\begin{tabular}{|c|c|c|c|c|c|c|c|}
\hline Number & Accession Number & Title & Date & $\begin{array}{l}\text { Source of Date } \\
\text { Information }\end{array}$ & Location & $\begin{array}{l}\text { Source of Location } \\
\text { Information }\end{array}$ & Themes \\
\hline 462 & W1995.030.334 & $\begin{array}{l}\text { Steamboating on the } \\
\text { Blackwater }\end{array}$ & $\begin{array}{l}\text { March 11, } \\
1863\end{array}$ & $\begin{array}{c}\text { WVRHC } \\
\text { Description Page }\end{array}$ & $\begin{array}{l}\text { Canaan Valley, VA } \\
\text { (WV) }\end{array}$ & On Drawing & $\begin{array}{l}\text { Men at Work, } \\
\text { Transportation } \\
\text { (Boats) }\end{array}$ \\
\hline 463 & W1995.030.234 & $\begin{array}{l}\text { Steamer Newcombe } \\
\text { Mississippi River-March } 1857\end{array}$ & March 1857 & On Drawing & Mississippi River & On Drawing & Men at Leisure \\
\hline 464 & W1995.030.387pg24a & $\begin{array}{l}\text { Steamer Newcombe above } \\
\text { Vicksburg Mississippi }\end{array}$ & $\begin{array}{l}\text { March 26, } \\
1857\end{array}$ & On Drawing & Vicksburg, Mississippi & On Drawing & Men at Leisure \\
\hline 465 & W1995.030.393pg26a & $\begin{array}{l}\text { Steamer Niagara Sunk } \\
\text { Mississippi } 1857 .\end{array}$ & 1857 & On Drawing & Mississippi & On Drawing & $\begin{array}{l}\text { African } \\
\text { Americans, } \\
\text { Men, } \\
\text { Transportation } \\
\text { (Boats) }\end{array}$ \\
\hline 466 & W1995.030.393pg12b & $\begin{array}{l}\text { Steamer Swallow..1857 March } \\
\text { 29th Schoolmaster N.C. North } \\
\text { Carolina }\end{array}$ & $\begin{array}{l}\text { March 29, } \\
\quad 1857\end{array}$ & On Drawing & North Carolina & On Drawing & $\begin{array}{l}\text { African } \\
\text { Americans, } \\
\text { Children, Men, } \\
\text { Women }\end{array}$ \\
\hline 467 & W1995.030.394pg3a & $\begin{array}{l}\text { Steps of Tammany Hall/Old } \\
\text { Dodge/Sweating }\end{array}$ & Unknown & $\begin{array}{l}\text { No Date on } \\
\text { Drawing }\end{array}$ & Unknown & No Location on Drawing & Children, Men \\
\hline 468 & W1995.030.393pg34b & $\begin{array}{l}\text { Stookey (sketch bears a } \\
\text { person's name and address) }\end{array}$ & Unknown & $\begin{array}{l}\text { No Date on } \\
\text { Drawing }\end{array}$ & Unknown & No Location on Drawing & Men \\
\hline 469 & W1995.030.048 & $\begin{array}{l}\text { Storming of the Engine House } \\
\text { at Harper's Ferry/ Capture of } \\
\text { John Brown }\end{array}$ & $\begin{array}{l}\text { October/ } \\
\text { November } \\
1859\end{array}$ & On Drawing & $\begin{array}{l}\text { Harpers Ferry, VA } \\
\text { (WV) }\end{array}$ & On Drawing & $\begin{array}{l}\text { Architecture } \\
\text { (Institutional } \\
\text { Buildings), } \\
\text { Harper's Ferry } \\
\text { Raid Trial, } \\
\text { Men, Military }\end{array}$ \\
\hline 470 & W1995.030.009 & Sturgeon- 8 feet long & May 5,1859 & On Drawing & Unknown & No Location on Drawing & Animals (Wild) \\
\hline
\end{tabular}




\begin{tabular}{|c|c|c|c|c|c|c|c|}
\hline Number & Accession Number & Title & Date & $\begin{array}{l}\text { Source of Date } \\
\text { Information }\end{array}$ & Location & $\begin{array}{l}\text { Source of Location } \\
\text { Information }\end{array}$ & Themes \\
\hline 471 & W1995.030.128 & Sugar House Louisiana & $1870 \mathrm{~s}$ & $\begin{array}{c}\text { WVRHC } \\
\text { Description Page }\end{array}$ & Louisiana & On Drawing & $\begin{array}{l}\text { Architecture, } \\
\text { Industry, } \\
\text { Landscape }\end{array}$ \\
\hline 472 & W1995.030.145 & $\begin{array}{l}\text { Sugar House and Refinery on } \\
\text { the Bayou Teche, St. Mary's } \\
\text { Parish, La. }\end{array}$ & Unknown & $\begin{array}{l}\text { No Date on } \\
\text { Drawing }\end{array}$ & $\begin{array}{l}\text { Bayou Teche, St. } \\
\text { Mary's Parish, LA }\end{array}$ & On Drawing & $\begin{array}{l}\text { Animals } \\
\text { (Domestic), } \\
\text { Architecture, } \\
\text { Industry, } \\
\text { Landscape, } \\
\text { Men at Work, } \\
\text { Transportation } \\
\text { (Carriages) }\end{array}$ \\
\hline 473 & W1995.030.139 & Sugar House. Bayou tache La. & $\begin{array}{l}\text { October 20, } \\
\quad 1853\end{array}$ & $\begin{array}{c}\text { WVRHC } \\
\text { Description Page }\end{array}$ & $\begin{array}{l}\text { Bayou Teche, St. } \\
\text { Mary's Parish, LA }\end{array}$ & On Drawing & $\begin{array}{l}\text { Architecture, } \\
\text { Industry, } \\
\text { Landscape, } \\
\text { Men at Work }\end{array}$ \\
\hline 474 & W1995.030.127 & Sugar Plantation Louisiana & $\begin{array}{l}\text { March 18, } \\
\quad 1857\end{array}$ & On Drawing & Louisiana & On Drawing & $\begin{array}{l}\text { African } \\
\text { Americans, } \\
\text { Architecture } \\
\text { (Residential } \\
\text { Architecture) }\end{array}$ \\
\hline 475 & W1995.030.394pg18 & $\begin{array}{l}\text { Summit House, Mt. } \\
\text { Washington }\end{array}$ & June 25,1859 & On Drawing & Mt. Washington, NH & On Drawing & $\begin{array}{l}\text { Animals } \\
\text { (Domestic), } \\
\text { Architecture, } \\
\text { Landscape } \\
\text { (Mountains) }\end{array}$ \\
\hline 476 & W1995.030.158 & $\begin{array}{l}\text { Summit of the Roane Distant } \\
\text { view of the Grandfather and } \\
\text { Yellow Mountain }\end{array}$ & $\begin{array}{c}\text { December } 4 \\
1856\end{array}$ & On Drawing & Unknown & No Location on Drawing & $\begin{array}{l}\text { Landscape } \\
\text { (Mountains), } \\
\text { Men at Leisure, } \\
\text { Men in } \\
\text { Wilderness }\end{array}$ \\
\hline 477 & W1995.030.131 & $\begin{array}{l}\text { Swamp Near New Orleans. } \\
\text { Lake Ponchartrain Feb. 24th } \\
1857\end{array}$ & $\begin{array}{c}\text { February } 24 \\
1857\end{array}$ & On Drawing & New Orleans, LA & On Drawing & Landscape \\
\hline
\end{tabular}




\begin{tabular}{|c|c|c|c|c|c|c|c|}
\hline Number & Accession Number & Title & Date & $\begin{array}{l}\text { Source of Date } \\
\text { Information }\end{array}$ & Location & $\begin{array}{l}\text { Source of Location } \\
\text { Information }\end{array}$ & Themes \\
\hline 478 & W1995.030.393pg12a & Sweet Mississipi Steamer. & $\begin{array}{l}\text { August 14, } \\
1858\end{array}$ & $\begin{array}{c}\text { WVRHC } \\
\text { Description Page }\end{array}$ & Unknown & No Location on Drawing & Men at Leisure \\
\hline 479 & W1995.030.394pg22 & $\begin{array}{l}\text { Taken at Harpers Ferry, Oct. } \\
18,1859 \text { during his } \\
\text { examination by Gov. Wise. } \\
\text { John Brown aged } 60 \text { years }\end{array}$ & $\begin{array}{l}\text { October } 18 \\
1859\end{array}$ & On Drawing & Martinsburg, VA (WV) & On Drawing & $\begin{array}{l}\text { Harper's Ferry } \\
\text { Raid Trial, } \\
\text { Men }\end{array}$ \\
\hline 480 & W1995.030.393pg23a & Tho's Jeffersons Statue & $\begin{array}{l}\text { March 30 } \\
\text { (Year } \\
\text { Unknown) }\end{array}$ & $\begin{array}{c}\text { WVRHC } \\
\text { Description Page }\end{array}$ & Unknown & No Location on Drawing & $\begin{array}{l}\text { African } \\
\text { Americans, } \\
\text { Women }\end{array}$ \\
\hline 481 & W1995.030.393pg11a & $\begin{array}{l}\text { Three naked women sculpted } \\
\text { by Hiram Powers. } 6 \text { legged } \\
\text { cow } 5 \text { legged sheep }\end{array}$ & Unknown & $\begin{array}{l}\text { No Date on } \\
\text { Drawing }\end{array}$ & Unknown & No Location on Drawing & $\begin{array}{l}\text { African } \\
\text { Americans, } \\
\text { Animals } \\
\text { (Domestic), } \\
\text { Men, Women }\end{array}$ \\
\hline 482 & W1995.030.393pg1a & $\begin{array}{l}\text { Tiles. Jordan Drummer } \\
\text { Tossing Hats overboard }\end{array}$ & $\begin{array}{l}\text { May 14, } \\
\text { 1859/ May } \\
16,1859 / \\
\text { May 19, } 1859\end{array}$ & $\begin{array}{c}\text { WVRHC } \\
\text { Description Page }\end{array}$ & Unknown & No Location on Drawing & $\begin{array}{l}\text { Men at Leisure, } \\
\text { Transportation } \\
\text { (Boats) }\end{array}$ \\
\hline 483 & W1995.030.393pg10b & $\begin{array}{l}\text { To B. Mayer, Charles Jr. } \\
\text { Mayer Baltimore }\end{array}$ & 1881 & $\begin{array}{c}\text { WVRHC } \\
\text { Description Page }\end{array}$ & Baltimore, MD & On Drawing & Men \\
\hline 484 & W1995.030.215 & Tom Athy/Watching & $\begin{array}{l}\text { September } \\
20,1846\end{array}$ & $\begin{array}{c}\text { WVRHC } \\
\text { Description Page }\end{array}$ & Unknown & No Location on Drawing & $\begin{array}{l}\text { Animals } \\
\text { (Domestic), } \\
\text { Animals } \\
\text { (Wild), } \\
\text { Landscape } \\
\text { (Mountains), } \\
\text { Men at Leisure, } \\
\text { Men in } \\
\text { Wilderness }\end{array}$ \\
\hline
\end{tabular}




\begin{tabular}{|c|c|c|c|c|c|c|c|}
\hline Number & Accession Number & Title & Date & $\begin{array}{l}\text { Source of Date } \\
\text { Information }\end{array}$ & Location & $\begin{array}{l}\text { Source of Location } \\
\text { Information }\end{array}$ & Themes \\
\hline 485 & W1995.030.007 & Tomb of Gov. Walker & $\begin{array}{l}\text { April 14, } \\
1856\end{array}$ & On Drawing & Unknown & No Location on Drawing & $\begin{array}{l}\text { Cemeteries, } \\
\text { Landscape } \\
\text { (Rivers), } \\
\text { Transportation } \\
\text { (Boats) }\end{array}$ \\
\hline 486 & W1995.030.392pg7b & $\begin{array}{l}\text { Tomb of John Custis of } \\
\text { Arlington. Northhampton Co: } \\
\text { Va May 6th } 1887\end{array}$ & May 6, 1887 & On Drawing & Arlington, VA & On Drawing & $\begin{array}{l}\text { Cemeteries, } \\
\text { Landscape } \\
\text { (Rivers), Men } \\
\text { at Leisure, } \\
\text { Transportation } \\
\text { (Boats) }\end{array}$ \\
\hline 487 & W1995.030.395pg11 & $\begin{array}{l}\text { Tommy Howard Decem: 29th } \\
1874 \text { at Norborne }\end{array}$ & $\begin{array}{c}\text { December } 29, \\
1874\end{array}$ & On Drawing & Martinsburg, VA (WV) & $\begin{array}{l}\text { Cecil Eby, "Porte } \\
\text { Crayon": The Life of } \\
\text { David Hunter Strother, } \\
\text { (Chapel Hill, NC: } \\
\text { University of North } \\
\text { Carolina Press, 1960), } 62 .\end{array}$ & $\begin{array}{l}\text { Children, } \\
\text { Decorative } \\
\text { Arts }\end{array}$ \\
\hline 488 & W1995.030.061 & $\begin{array}{l}\text { Topographical sketch of the } \\
\text { Vicinity of Liberty Mills }\end{array}$ & Unknown & $\begin{array}{l}\text { No Date on } \\
\text { Drawing }\end{array}$ & Unknown & No Location on Drawing & Military \\
\hline 489 & W1995.030.184 & Trying the Drum & Unknown & $\begin{array}{l}\text { No Date on } \\
\text { Drawing }\end{array}$ & Unknown & No Location on Drawing & $\begin{array}{l}\text { Animals } \\
\text { (Domestic), } \\
\text { Men, Military }\end{array}$ \\
\hline 490 & W1995.030.319 & $\begin{array}{l}\text { Tunnel of Sandy, visited by R. } \\
\text { Harrison and DH Strother } \\
\text { October 1st, } 1860\end{array}$ & $\begin{array}{c}\text { October } 1 \\
1860\end{array}$ & On Drawing & Unknown & No Location on Drawing & $\begin{array}{l}\text { Landscape } \\
\text { (Cave) }\end{array}$ \\
\hline 491 & W1995.030.072 & $\begin{array}{l}\text { United States forces at } \\
\text { Edwards ferry Va. }\end{array}$ & 1852 & $\begin{array}{c}\text { WVRHC } \\
\text { Description Page }\end{array}$ & Edwards Ferry, VA & On Drawing & $\begin{array}{l}\text { Landscape, } \\
\text { Military }\end{array}$ \\
\hline
\end{tabular}




\begin{tabular}{|c|c|c|c|c|c|c|c|}
\hline Number & Accession Number & Title & Date & $\begin{array}{l}\text { Source of Date } \\
\text { Information }\end{array}$ & Location & $\begin{array}{l}\text { Source of Location } \\
\text { Information }\end{array}$ & Themes \\
\hline 492 & W1995.030.002 & Untitled & $\begin{array}{c}\text { September } \\
1849\end{array}$ & On Drawing & Unknown & No Location on Drawing & Men at Work \\
\hline 493 & W1995.030.005 & Untitled & $\begin{array}{c}\text { February } \\
1859\end{array}$ & On Drawing & Unknown & No Location on Drawing & Men at Leisure \\
\hline 494 & W1995.030.039 & Untitled & $\begin{array}{l}\text { November } \\
28,1876\end{array}$ & On Drawing & Unknown & No Location on Drawing & $\begin{array}{l}\text { Animals } \\
\text { (Domestic) }\end{array}$ \\
\hline 495 & W1995.030.058 & Untitled & 1852 & $\begin{array}{c}\text { WVRHC } \\
\text { Description Page }\end{array}$ & Unknown & No Location on Drawing & $\begin{array}{l}\text { Animals } \\
\text { (Domestic), } \\
\text { Men, Military }\end{array}$ \\
\hline 496 & W1995.030.073 & Untitled & Unknown & $\begin{array}{l}\text { No Date on } \\
\text { Drawing }\end{array}$ & Unknown & No Location on Drawing & Men, Military \\
\hline 497 & W1995.030.079 & Untitled & June 1852 & $\begin{array}{c}\text { WVRHC } \\
\text { Description Page }\end{array}$ & Unknown & No Location on Drawing & $\begin{array}{l}\text { Decorative } \\
\text { Arts, Men at } \\
\text { Work }\end{array}$ \\
\hline 498 & W1995.030.081 & Untitled & $\begin{array}{l}\text { December 2, } \\
1859\end{array}$ & $\begin{array}{c}\text { WVRHC } \\
\text { Description Page }\end{array}$ & Unknown & No Location on Drawing & $\begin{array}{l}\text { Architecture, } \\
\text { Decorative } \\
\text { Arts, } \\
\text { Landscape, } \\
\text { Women at } \\
\text { Work }\end{array}$ \\
\hline 499 & W1995.030.087 & Untitled & Unknown & $\begin{array}{l}\text { No Date on } \\
\text { Drawing }\end{array}$ & Unknown & No Location on Drawing & $\begin{array}{l}\text { Architecture, } \\
\text { Women }\end{array}$ \\
\hline 500 & W1995.030.094 & Untitled & Unknown & $\begin{array}{l}\text { No Date on } \\
\text { Drawing }\end{array}$ & Italy & $\begin{array}{c}\text { WVRHC Description } \\
\text { Page }\end{array}$ & $\begin{array}{l}\text { Architecture, } \\
\text { Landscape } \\
\text { (Mountains) }\end{array}$ \\
\hline
\end{tabular}




\begin{tabular}{|c|c|c|c|c|c|c|c|}
\hline Number & Accession Number & Title & Date & $\begin{array}{l}\text { Source of Date } \\
\text { Information }\end{array}$ & Location & $\begin{array}{l}\text { Source of Location } \\
\text { Information }\end{array}$ & Themes \\
\hline 501 & W1995.030.101 & Untitled & Unknown & $\begin{array}{l}\text { No Date on } \\
\text { Drawing }\end{array}$ & Italy & $\begin{array}{c}\text { WVRHC Description } \\
\text { Page }\end{array}$ & $\begin{array}{l}\text { Architecture } \\
\text { (Residential } \\
\text { Buildings), } \\
\text { Architecture } \\
\text { (Towns), } \\
\text { Landscape } \\
\text { (Mountains) }\end{array}$ \\
\hline 502 & W1995.030.103 & Untitled & $\begin{array}{l}\text { December } 9, \\
1856\end{array}$ & $\begin{array}{c}\text { WVRHC } \\
\text { Description Page }\end{array}$ & Italy & $\begin{array}{c}\text { WVRHC Description } \\
\text { Page }\end{array}$ & $\begin{array}{l}\text { Landscape } \\
\text { (Mountains), } \\
\text { Religious } \\
\text { Shrines }\end{array}$ \\
\hline 503 & W1995.030.104 & Untitled & Unknown & $\begin{array}{l}\text { No Date on } \\
\text { Drawing }\end{array}$ & Unknown & No Location on Drawing & $\begin{array}{l}\text { Architecture } \\
\text { (Towns), } \\
\text { Landscape }\end{array}$ \\
\hline 504 & W1995.030.105 & Untitled & Unknown & $\begin{array}{l}\text { No Date on } \\
\text { Drawing }\end{array}$ & Unknown & No Location on Drawing & $\begin{array}{l}\text { Landscape } \\
\text { (Caves), Men, } \\
\text { Transportation } \\
\text { (Boats) }\end{array}$ \\
\hline 505 & W1995.030.106 & Untitled & $\begin{array}{c}\text { February } 4, \\
1857\end{array}$ & $\begin{array}{c}\text { WVRHC } \\
\text { Description Page }\end{array}$ & Unknown & No Location on Drawing & Men at Leisure \\
\hline 506 & W1995.030.107 & Untitled & Unknown & $\begin{array}{l}\text { No Date on } \\
\text { Drawing }\end{array}$ & Unknown & No Location on Drawing & $\begin{array}{l}\text { Animals, } \\
\text { Architecture } \\
\text { (Towns), } \\
\text { Landscape } \\
\text { (Mountains), } \\
\text { Men, Women }\end{array}$ \\
\hline 507 & W1995.030.108 & Untitled & Unknown & $\begin{array}{l}\text { No Date on } \\
\text { Drawing }\end{array}$ & Unknown & No Location on Drawing & $\begin{array}{l}\text { Architecture, } \\
\text { Landscape }\end{array}$ \\
\hline
\end{tabular}




\begin{tabular}{|c|c|c|c|c|c|c|c|}
\hline Number & Accession Number & Title & Date & $\begin{array}{l}\text { Source of Date } \\
\text { Information }\end{array}$ & Location & $\begin{array}{l}\text { Source of Location } \\
\text { Information }\end{array}$ & Themes \\
\hline 508 & W1995.030.109 & Untitled & Unknown & $\begin{array}{l}\text { No Date on } \\
\text { Drawing }\end{array}$ & Italy & $\begin{array}{c}\text { WVRHC Description } \\
\text { Page }\end{array}$ & $\begin{array}{l}\text { Children, } \\
\text { Landscape } \\
\text { (Caves), Men, } \\
\text { Women }\end{array}$ \\
\hline 509 & W1995.030.110 & Untitled & $\begin{array}{c}\text { December } 24 \\
1860\end{array}$ & $\begin{array}{c}\text { WVRHC } \\
\text { Description Page }\end{array}$ & Unknown & No Location on Drawing & $\begin{array}{l}\text { Children, } \\
\text { Landscape } \\
\text { (Mountains), } \\
\text { Men, Religious } \\
\text { Shrines, } \\
\text { Women }\end{array}$ \\
\hline 510 & W1995.030.111 & Untitled & Unknown & $\begin{array}{l}\text { No Date on } \\
\text { Drawing }\end{array}$ & Florence, Italy & $\begin{array}{c}\text { WVRHC Description } \\
\text { Page }\end{array}$ & $\begin{array}{l}\text { Architecture } \\
\text { (Towns), } \\
\text { Decorative } \\
\text { Arts }\end{array}$ \\
\hline 511 & W1995.030.112 & Untitled & January 1873 & $\begin{array}{c}\text { WVRHC } \\
\text { Description Page }\end{array}$ & Unknown & No Location on Drawing & $\begin{array}{l}\text { Landscape } \\
\text { (Mountains) }\end{array}$ \\
\hline 512 & W1995.030.113 & Untitled & January 1873 & $\begin{array}{c}\text { WVRHC } \\
\text { Description Page }\end{array}$ & Unknown & No Location on Drawing & $\begin{array}{l}\text { Architecture } \\
\text { (Towns), } \\
\text { Landscape } \\
\text { (Lakes), } \\
\text { Landscape } \\
\text { (Mountains), } \\
\text { Men }\end{array}$ \\
\hline
\end{tabular}




\begin{tabular}{|c|c|c|c|c|c|c|c|}
\hline Number & Accession Number & Title & Date & $\begin{array}{l}\text { Source of Date } \\
\text { Information }\end{array}$ & Location & $\begin{array}{l}\text { Source of Location } \\
\text { Information }\end{array}$ & Themes \\
\hline 513 & W1995.030.114 & Untitled & $\begin{array}{c}\text { November } \\
1856\end{array}$ & $\begin{array}{c}\text { WVRHC } \\
\text { Description Page }\end{array}$ & Unknown & No Location on Drawing & $\begin{array}{l}\text { Architecture } \\
\text { (Bridges), } \\
\text { Architecture } \\
\text { (Towns), } \\
\text { Landscape } \\
\text { (Mountains), } \\
\text { Landscape } \\
\text { (Waterfalls) }\end{array}$ \\
\hline 514 & W1995.030.115 & Untitled & Unknown & $\begin{array}{l}\text { No Date on } \\
\text { Drawing }\end{array}$ & Unknown & No Location on Drawing & $\begin{array}{l}\text { Architecture } \\
\text { (Towns) }\end{array}$ \\
\hline 515 & W1995.030.116 & Untitled & $\begin{array}{c}\text { November } \\
15,1856\end{array}$ & $\begin{array}{c}\text { WVRHC } \\
\text { Description Page }\end{array}$ & Unknown & No Location on Drawing & $\begin{array}{l}\text { Architecture } \\
\text { (Towns), } \\
\text { Landscape }\end{array}$ \\
\hline 516 & W1995.030.117 & Untitled & $\begin{array}{l}\text { September } \\
30,1876\end{array}$ & $\begin{array}{c}\text { WVRHC } \\
\text { Description Page }\end{array}$ & Unknown & No Location on Drawing & $\begin{array}{l}\text { Decorative } \\
\text { Arts, } \\
\text { Landscape }\end{array}$ \\
\hline 517 & W1995.030.118 & Untitled & 1845 & $\begin{array}{c}\text { WVRHC } \\
\text { Description Page }\end{array}$ & Unknown & No Location on Drawing & Men \\
\hline 518 & W1995.030.119 & Untitled & May 6,1887 & $\begin{array}{c}\text { WVRHC } \\
\text { Description Page }\end{array}$ & Unknown & No Location on Drawing & $\begin{array}{l}\text { Architecture } \\
\text { (Towns), } \\
\text { Landscape }\end{array}$ \\
\hline 519 & W1995.030.120 & Untitled & Unknown & $\begin{array}{l}\text { No Date on } \\
\text { Drawing }\end{array}$ & Unknown & No Location on Drawing & $\begin{array}{l}\text { Copies of } \\
\text { European } \\
\text { Artwork }\end{array}$ \\
\hline
\end{tabular}




\begin{tabular}{|c|c|c|c|c|c|c|c|}
\hline Number & Accession Number & Title & Date & $\begin{array}{l}\text { Source of Date } \\
\text { Information }\end{array}$ & Location & $\begin{array}{l}\text { Source of Location } \\
\text { Information }\end{array}$ & Themes \\
\hline 520 & W1995.030.121 & Untitled & 1848 & $\begin{array}{c}\text { WVRHC } \\
\text { Description Page }\end{array}$ & Florence, Italy & $\begin{array}{c}\text { WVRHC Description } \\
\text { Page }\end{array}$ & $\begin{array}{l}\text { Architecture } \\
\text { (Towns), } \\
\text { Landscape }\end{array}$ \\
\hline 521 & W1995.030.122 & Untitled & $\begin{array}{l}\text { December } 5 \\
\quad 1856\end{array}$ & $\begin{array}{c}\text { WVRHC } \\
\text { Description Page }\end{array}$ & Unknown & No Location on Drawing & $\begin{array}{l}\text { Animals } \\
\text { (Domestic), } \\
\text { Landscape } \\
\text { (Mountains), } \\
\text { Landscape } \\
\text { (Waterfalls), } \\
\text { Men in } \\
\text { Wilderness }\end{array}$ \\
\hline 522 & W1995.030.123 & Untitled & Unknown & $\begin{array}{l}\text { No Date on } \\
\text { Drawing }\end{array}$ & Venice, Italy & $\begin{array}{c}\text { WVRHC Description } \\
\text { Page }\end{array}$ & $\begin{array}{l}\text { Architecture } \\
\text { (Towns), } \\
\text { Landscape } \\
\text { (Lakes), } \\
\text { Landscape } \\
\text { (Mountains) }\end{array}$ \\
\hline 523 & W1995.030.141 & Untitled & July 1874 & $\begin{array}{c}\text { WVRHC } \\
\text { Description Page }\end{array}$ & Unknown & No Location on Drawing & $\begin{array}{l}\text { Landscape } \\
\text { (Rivers), } \\
\text { Transportation } \\
\text { (Boats), } \\
\text { Transportation } \\
\text { (Carriages) }\end{array}$ \\
\hline 524 & W1995.030.143b & Untitled & $\begin{array}{l}\text { April 23, } \\
1887\end{array}$ & $\begin{array}{c}\text { WVRHC } \\
\text { Description Page }\end{array}$ & Unknown & No Location on Drawing & $\begin{array}{l}\text { Animals } \\
\text { (Domestic) }\end{array}$ \\
\hline 525 & W1995.030.150 & Untitled & Unknown & $\begin{array}{l}\text { No Date on } \\
\text { Drawing }\end{array}$ & Unknown & No Location on Drawing & $\begin{array}{l}\text { Landscape, } \\
\text { Women }\end{array}$ \\
\hline 526 & W1995.030.151b & Untitled & Unknown & $\begin{array}{l}\text { No Date on } \\
\text { Drawing }\end{array}$ & Unknown & No Location on Drawing & Men at Work \\
\hline
\end{tabular}




\begin{tabular}{|c|c|c|c|c|c|c|c|}
\hline Number & Accession Number & Title & Date & $\begin{array}{l}\text { Source of Date } \\
\text { Information }\end{array}$ & Location & $\begin{array}{l}\text { Source of Location } \\
\text { Information }\end{array}$ & Themes \\
\hline 527 & W1995.030.152b & Untitled & Unknown & $\begin{array}{l}\text { No Date on } \\
\text { Drawing }\end{array}$ & Unknown & No Location on Drawing & $\begin{array}{l}\text { Animals, Men } \\
\text { at Leisure }\end{array}$ \\
\hline 528 & W1995.030.153 & Untitled & Unknown & $\begin{array}{l}\text { No Date on } \\
\text { Drawing }\end{array}$ & Mexico City, Mexico & $\begin{array}{c}\text { WVRHC Description } \\
\text { Page }\end{array}$ & Men \\
\hline 529 & W1995.030.161b & Untitled & Unknown & $\begin{array}{l}\text { No Date on } \\
\text { Drawing }\end{array}$ & Unknown & No Location on Drawing & Men, Military \\
\hline 530 & W1995.030.165b & Untitled & May 3, 1879 & $\begin{array}{c}\text { WVRHC } \\
\text { Description Page }\end{array}$ & Unknown & No Location on Drawing & $\begin{array}{l}\text { Animals, } \\
\text { Children }\end{array}$ \\
\hline 531 & W1995.030.167 & Untitled & $\begin{array}{l}\text { April 29, } \\
1887\end{array}$ & $\begin{array}{c}\text { WVRHC } \\
\text { Description Page }\end{array}$ & Unknown & No Location on Drawing & $\begin{array}{l}\text { Men at Work, } \\
\text { Landscape } \\
\text { (Rivers) }\end{array}$ \\
\hline 532 & W1995.030.172b & Untitled & $\begin{array}{c}\text { November } \\
23,1856\end{array}$ & $\begin{array}{c}\text { WVRHC } \\
\text { Description Page }\end{array}$ & Unknown & No Location on Drawing & $\begin{array}{l}\text { Industry, Men } \\
\text { at Work }\end{array}$ \\
\hline 533 & W1995.030.183 & Untitled & Unknown & $\begin{array}{l}\text { No Date on } \\
\text { Drawing }\end{array}$ & Unknown & No Location on Drawing & Children \\
\hline 534 & W1995.030.185 & Untitled & Unknown & $\begin{array}{l}\text { No Date on } \\
\text { Drawing }\end{array}$ & Unknown & No Location on Drawing & $\begin{array}{l}\text { African } \\
\text { Americans, } \\
\text { Children, Men, } \\
\text { Military }\end{array}$ \\
\hline 535 & W1995.030.186 & Untitled & $\begin{array}{c}\text { December } \\
1867\end{array}$ & $\begin{array}{c}\text { WVRHC } \\
\text { Description Page }\end{array}$ & Unknown & No Location on Drawing & Children \\
\hline
\end{tabular}




\begin{tabular}{|c|c|c|c|c|c|c|c|}
\hline Number & Accession Number & Title & Date & $\begin{array}{l}\text { Source of Date } \\
\text { Information }\end{array}$ & Location & $\begin{array}{l}\text { Source of Location } \\
\text { Information }\end{array}$ & Themes \\
\hline 536 & W1995.030.187 & Untitled & $\begin{array}{c}\text { December } \\
1867\end{array}$ & $\begin{array}{c}\text { WVRHC } \\
\text { Description Page }\end{array}$ & Unknown & No Location on Drawing & $\begin{array}{l}\text { Animals } \\
\text { (Domestic), } \\
\text { Architecture } \\
\text { (Residential } \\
\text { Buildings), } \\
\text { Landscape, } \\
\text { Men at Work, } \\
\text { Women at } \\
\text { Work }\end{array}$ \\
\hline 537 & W1995.030.188 & Untitled & Unknown & $\begin{array}{l}\text { No Date on } \\
\text { Drawing }\end{array}$ & Unknown & No Location on Drawing & $\begin{array}{l}\text { African } \\
\text { Americans, } \\
\text { Children }\end{array}$ \\
\hline 538 & W1995.030.189 & Untitled & Unknown & $\begin{array}{l}\text { No Date on } \\
\text { Drawing }\end{array}$ & Unknown & No Location on Drawing & $\begin{array}{l}\text { Animals } \\
\text { (Domestic), } \\
\text { Children, Men, } \\
\text { Women }\end{array}$ \\
\hline 539 & W1995.030.190 & Untitled & Unknown & $\begin{array}{l}\text { No Date on } \\
\text { Drawing }\end{array}$ & Unknown & No Location on Drawing & $\begin{array}{l}\text { Children, } \\
\text { Landscape } \\
\text { (Fences) }\end{array}$ \\
\hline 540 & W1995.030.191 & Untitled & Unknown & $\begin{array}{l}\text { No Date on } \\
\text { Drawing }\end{array}$ & Unknown & No Location on Drawing & $\begin{array}{l}\text { Landscape } \\
\text { (Mountains), } \\
\text { Men at Leisure, } \\
\text { Transportation } \\
\text { (Carriages), } \\
\text { Women at } \\
\text { Leisure }\end{array}$ \\
\hline 541 & W1995.030.195 & Untitled & 1842 & $\begin{array}{c}\text { WVRHC } \\
\text { Description Page }\end{array}$ & Unknown & No Location on Drawing & $\begin{array}{l}\text { Landscape } \\
\text { (Mountains), } \\
\text { Men at Leisure, } \\
\text { Women at } \\
\text { Leisure }\end{array}$ \\
\hline 542 & W1995.030.196 & Untitled & Unknown & $\begin{array}{l}\text { No Date on } \\
\text { Drawing }\end{array}$ & Unknown & No Location on Drawing & $\begin{array}{l}\text { Landscape } \\
\text { (Rivers) }\end{array}$ \\
\hline
\end{tabular}




\begin{tabular}{|c|c|c|c|c|c|c|c|}
\hline Number & Accession Number & Title & Date & $\begin{array}{l}\text { Source of Date } \\
\text { Information }\end{array}$ & Location & $\begin{array}{l}\text { Source of Location } \\
\text { Information }\end{array}$ & Themes \\
\hline 543 & W1995.030.197 & Untitled & 1845 & $\begin{array}{c}\text { WVRHC } \\
\text { Description Page }\end{array}$ & Unknown & No Location on Drawing & $\begin{array}{l}\text { Animals } \\
\text { (Domestic) }\end{array}$ \\
\hline 544 & W1995.030.200 & Untitled & Unknown & $\begin{array}{l}\text { No Date on } \\
\text { Drawing }\end{array}$ & Unknown & No Location on Drawing & $\begin{array}{l}\text { Children, } \\
\text { Landscape } \\
\text { (Mountains), } \\
\text { Men at Leisure, } \\
\text { Women at } \\
\text { Leisure }\end{array}$ \\
\hline 545 & W1995.030.201 & Untitled & Unknown & $\begin{array}{l}\text { No Date on } \\
\text { Drawing }\end{array}$ & Unknown & No Location on Drawing & Men \\
\hline 546 & W1995.030.202a & Untitled & Unknown & $\begin{array}{l}\text { No Date on } \\
\text { Drawing }\end{array}$ & Unknown & No Location on Drawing & Men \\
\hline 547 & W1995.030.202b & Untitled & Unknown & $\begin{array}{l}\text { No Date on } \\
\text { Drawing }\end{array}$ & Unknown & No Location on Drawing & Men \\
\hline 548 & W1995.030.203 & Untitled & Unknown & $\begin{array}{l}\text { No Date on } \\
\text { Drawing }\end{array}$ & Unknown & No Location on Drawing & $\begin{array}{l}\text { Animals } \\
\text { (Domestic), } \\
\text { Animals } \\
\text { (Wild), } \\
\text { Children, Men } \\
\text { at Leisure }\end{array}$ \\
\hline 549 & W1995.030.204a & Untitled & Unknown & $\begin{array}{l}\text { No Date on } \\
\text { Drawing }\end{array}$ & Unknown & No Location on Drawing & $\begin{array}{l}\text { Animals } \\
\text { (Domestic), } \\
\text { Women }\end{array}$ \\
\hline 550 & W1995.030.204b & Untitled & Unknown & $\begin{array}{l}\text { No Date on } \\
\text { Drawing }\end{array}$ & Unknown & No Location on Drawing & $\begin{array}{l}\text { Animals, } \\
\text { Children, Men, } \\
\text { Women }\end{array}$ \\
\hline 551 & W1995.030.205 & Untitled & Unknown & $\begin{array}{l}\text { No Date on } \\
\text { Drawing }\end{array}$ & Unknown & No Location on Drawing & $\begin{array}{l}\text { African } \\
\text { Americans, } \\
\text { Children, } \\
\text { Women }\end{array}$ \\
\hline
\end{tabular}




\begin{tabular}{|c|c|c|c|c|c|c|c|}
\hline Number & Accession Number & Title & Date & $\begin{array}{l}\text { Source of Date } \\
\text { Information }\end{array}$ & Location & $\begin{array}{l}\text { Source of Location } \\
\text { Information }\end{array}$ & Themes \\
\hline 552 & W1995.030.206b & Untitled & Unknown & $\begin{array}{l}\text { No Date on } \\
\text { Drawing }\end{array}$ & Unknown & No Location on Drawing & $\begin{array}{l}\text { African } \\
\text { Americans, } \\
\text { Landscape } \\
\text { (Fences), Men } \\
\text { at Leisure }\end{array}$ \\
\hline 553 & W1995.030.207 & Untitled & Unknown & $\begin{array}{l}\text { No Date on } \\
\text { Drawing }\end{array}$ & Unknown & No Location on Drawing & Men \\
\hline 554 & W1995.030.208 & Untitled & Unknown & $\begin{array}{l}\text { No Date on } \\
\text { Drawing }\end{array}$ & Unknown & No Location on Drawing & $\begin{array}{l}\text { Architecture } \\
\text { (Residential } \\
\text { Buildings), } \\
\text { Landscape }\end{array}$ \\
\hline 555 & W1995.030.209 & Untitled & Unknown & $\begin{array}{l}\text { No Date on } \\
\text { Drawing }\end{array}$ & Unknown & No Location on Drawing & $\begin{array}{l}\text { Landscape } \\
\text { (Mountains), } \\
\text { Men at Leisure, } \\
\text { Women at } \\
\text { Leisure }\end{array}$ \\
\hline 556 & W1995.030.210 & Untitled & May 5,1862 & $\begin{array}{c}\text { WVRHC } \\
\text { Description Page }\end{array}$ & Unknown & No Location on Drawing & Men \\
\hline 557 & W1995.030.211 & Untitled & Unknown & $\begin{array}{l}\text { No Date on } \\
\text { Drawing }\end{array}$ & Unknown & No Location on Drawing & \\
\hline 558 & W1995.030.212 & Untitled & Unknown & $\begin{array}{l}\text { No Date on } \\
\text { Drawing }\end{array}$ & Unknown & No Location on Drawing & $\begin{array}{l}\text { Men at Work, } \\
\text { Military }\end{array}$ \\
\hline 559 & W1995.030.213 & Untitled & Unknown & $\begin{array}{l}\text { No Date on } \\
\text { Drawing }\end{array}$ & Unknown & No Location on Drawing & Women \\
\hline 560 & W1995.030.214 & Untitled & Unknown & $\begin{array}{l}\text { No Date on } \\
\text { Drawing }\end{array}$ & Unknown & No Location on Drawing & Landscape \\
\hline
\end{tabular}




\begin{tabular}{|c|c|c|c|c|c|c|c|}
\hline Number & Accession Number & Title & Date & $\begin{array}{l}\text { Source of Date } \\
\text { Information }\end{array}$ & Location & $\begin{array}{l}\text { Source of Location } \\
\text { Information }\end{array}$ & Themes \\
\hline 561 & W1995.030.216 & Untitled & Unknown & $\begin{array}{l}\text { No Date on } \\
\text { Drawing }\end{array}$ & Unknown & No Location on Drawing & $\begin{array}{l}\text { African } \\
\text { Americans, } \\
\text { Women }\end{array}$ \\
\hline 562 & W1995.030.218 & Untitled & 1859 & $\begin{array}{c}\text { WVRHC } \\
\text { Description Page }\end{array}$ & Unknown & No Location on Drawing & $\begin{array}{l}\text { Landscape } \\
\text { (Rivers), } \\
\text { Transportation } \\
\text { (Boats) }\end{array}$ \\
\hline 563 & W1995.030.219 & Untitled & Unknown & $\begin{array}{l}\text { No Date on } \\
\text { Drawing }\end{array}$ & Unknown & No Location on Drawing & Women \\
\hline 564 & W1995.030.220 & Untitled & Unknown & $\begin{array}{l}\text { No Date on } \\
\text { Drawing }\end{array}$ & Unknown & No Location on Drawing & $\begin{array}{l}\text { Landscape } \\
\text { (Mountains) }\end{array}$ \\
\hline 565 & W1995.030.221b & Untitled & Unknown & $\begin{array}{l}\text { No Date on } \\
\text { Drawing }\end{array}$ & Unknown & No Location on Drawing & \\
\hline 566 & W1995.030.222a & Untitled & $\begin{array}{l}\text { October 6, } \\
1854\end{array}$ & $\begin{array}{c}\text { WVRHC } \\
\text { Description Page }\end{array}$ & Unknown & No Location on Drawing & $\begin{array}{l}\text { Animals } \\
\text { (Domestic), } \\
\text { Men at Work, } \\
\text { Transportation } \\
\text { (Boats) }\end{array}$ \\
\hline 567 & W1995.030.222b & Untitled & Unknown & $\begin{array}{l}\text { No Date on } \\
\text { Drawing }\end{array}$ & Unknown & No Location on Drawing & Men \\
\hline 568 & W1995.030.223 & Untitled & May 6,1887 & $\begin{array}{c}\text { WVRHC } \\
\text { Description Page }\end{array}$ & Unknown & No Location on Drawing & $\begin{array}{l}\text { Animals } \\
\text { (Wild), } \\
\text { Landscape }\end{array}$ \\
\hline 569 & W1995.030.225 & Untitled & Unknown & $\begin{array}{l}\text { No Date on } \\
\text { Drawing }\end{array}$ & Unknown & No Location on Drawing & $\begin{array}{l}\text { Animals } \\
\text { (Domestic), } \\
\text { Transportation } \\
\text { (Carriages) }\end{array}$ \\
\hline
\end{tabular}




\begin{tabular}{|c|c|c|c|c|c|c|c|}
\hline Number & Accession Number & Title & Date & $\begin{array}{l}\text { Source of Date } \\
\text { Information }\end{array}$ & Location & $\begin{array}{l}\text { Source of Location } \\
\text { Information }\end{array}$ & Themes \\
\hline 570 & W1995.030.226b & Untitled & Unknown & $\begin{array}{l}\text { No Date on } \\
\text { Drawing }\end{array}$ & Unknown & No Location on Drawing & $\begin{array}{l}\text { Architecture } \\
\text { (Institutional } \\
\text { Buildings) }\end{array}$ \\
\hline 571 & W1995.030.228 & Untitled & Unknown & $\begin{array}{l}\text { No Date on } \\
\text { Drawing }\end{array}$ & Unknown & No Location on Drawing & Men \\
\hline 572 & W1995.030.230 & Untitled & $\begin{array}{c}\text { February } \\
1859\end{array}$ & On Drawing & Unknown & No Location on Drawing & Men at Leisure \\
\hline 573 & W1995.030.231 & Untitled & Unknown & $\begin{array}{l}\text { No Date on } \\
\text { Drawing }\end{array}$ & Unknown & No Location on Drawing & Men \\
\hline 574 & W1995.030.232b & Untitled & Unknown & $\begin{array}{l}\text { No Date on } \\
\text { Drawing }\end{array}$ & Unknown & No Location on Drawing & Men, Women \\
\hline 575 & W1995.030.235 & Untitled & $\begin{array}{c}\text { December } 9 \\
1856\end{array}$ & $\begin{array}{c}\text { WVRHC } \\
\text { Description Page }\end{array}$ & Unknown & No Location on Drawing & Men \\
\hline 576 & W1995.030.238 & Untitled & $\begin{array}{c}\text { December } 15 \\
1867\end{array}$ & $\begin{array}{c}\text { WVRHC } \\
\text { Description Page }\end{array}$ & Unknown & No Location on Drawing & Men \\
\hline 577 & W1995.030.239a & Untitled & $\begin{array}{c}\text { December } 15, \\
1867\end{array}$ & $\begin{array}{c}\text { WVRHC } \\
\text { Description Page }\end{array}$ & Unknown & No Location on Drawing & $\begin{array}{l}\text { African } \\
\text { Americans, } \\
\text { Animals } \\
\text { (Domestic), } \\
\text { Children }\end{array}$ \\
\hline 578 & W1995.030.239b & Untitled & Unknown & $\begin{array}{l}\text { No Date on } \\
\text { Drawing }\end{array}$ & Unknown & No Location on Drawing & $\begin{array}{l}\text { Architecture } \\
\text { (Residential } \\
\text { Buildings), } \\
\text { Landscape } \\
\text { (Fences), } \\
\text { Women at } \\
\text { Leisure }\end{array}$ \\
\hline
\end{tabular}




\begin{tabular}{|c|c|c|c|c|c|c|c|}
\hline Number & Accession Number & Title & Date & $\begin{array}{l}\text { Source of Date } \\
\text { Information }\end{array}$ & Location & $\begin{array}{l}\text { Source of Location } \\
\text { Information }\end{array}$ & Themes \\
\hline 579 & W1995.030.242b & Untitled & Unknown & $\begin{array}{c}\text { No Date on } \\
\text { Drawing }\end{array}$ & Unknown & No Location on Drawing & Men \\
\hline 580 & W1995.030.243 & Untitled & $\begin{array}{c}\text { October } 8 \\
1854\end{array}$ & $\begin{array}{c}\text { WVRHC } \\
\text { Description Page }\end{array}$ & Unknown & No Location on Drawing & Children \\
\hline 581 & W1995.030.244b & Untitled & Unknown & $\begin{array}{l}\text { No Date on } \\
\text { Drawing }\end{array}$ & Unknown & No Location on Drawing & Women \\
\hline 582 & W1995.030.245 & Untitled & Unknown & $\begin{array}{l}\text { No Date on } \\
\text { Drawing }\end{array}$ & Unknown & No Location on Drawing & Men at Leisure \\
\hline 583 & W1995.030.246 & Untitled & Unknown & $\begin{array}{l}\text { No Date on } \\
\text { Drawing }\end{array}$ & Unknown & No Location on Drawing & Women \\
\hline 584 & W1995.030.248a & Untitled & Unknown & $\begin{array}{l}\text { No Date on } \\
\text { Drawing }\end{array}$ & Unknown & No Location on Drawing & $\begin{array}{l}\text { Animals } \\
\text { (Domestic), } \\
\text { Architecture } \\
\text { (Residential } \\
\text { Buildings), } \\
\text { Children, } \\
\text { Women }\end{array}$ \\
\hline 585 & W1995.030.249b & Untitled & $\begin{array}{c}\text { August 16, } \\
1859\end{array}$ & $\begin{array}{c}\text { WVRHC } \\
\text { Description Page }\end{array}$ & Unknown & No Location on Drawing & $\begin{array}{l}\text { Animals } \\
\text { (Domestic) }\end{array}$ \\
\hline 586 & W1995.030.256b & Untitled & $\begin{array}{c}\text { September } \\
25,1860\end{array}$ & $\begin{array}{c}\text { WVRHC } \\
\text { Description Page }\end{array}$ & Unknown & No Location on Drawing & $\begin{array}{l}\text { Children, } \\
\text { Women }\end{array}$ \\
\hline 587 & W1995.030.258 & Untitled & $\begin{array}{c}\text { October } 9 \\
1854\end{array}$ & $\begin{array}{c}\text { WVRHC } \\
\text { Description Page }\end{array}$ & Unknown & No Location on Drawing & Children \\
\hline 588 & W1995.030.263 & Untitled & $\begin{array}{l}\text { March 4, } \\
1865\end{array}$ & On Drawing & Unknown & No Location on Drawing & Women \\
\hline 589 & W1995.030.272 & Untitled & $\begin{array}{l}\text { March 4, } \\
1856\end{array}$ & On Drawing & Unknown & No Location on Drawing & Men \\
\hline
\end{tabular}




\begin{tabular}{|c|c|c|c|c|c|c|c|}
\hline Number & Accession Number & Title & Date & $\begin{array}{l}\text { Source of Date } \\
\text { Information }\end{array}$ & Location & $\begin{array}{l}\text { Source of Location } \\
\text { Information }\end{array}$ & Themes \\
\hline 590 & W1995.030.273 & Untitled & May 20,1859 & $\begin{array}{c}\text { WVRHC } \\
\text { Description Page }\end{array}$ & Unknown & No Location on Drawing & Men \\
\hline 591 & W1995.030.275 & Untitled & June 25,1859 & $\begin{array}{c}\text { WVRHC } \\
\text { Description Page }\end{array}$ & Unknown & No Location on Drawing & Women \\
\hline 592 & W1995.030.281 & Untitled & Unknown & $\begin{array}{l}\text { No Date on } \\
\text { Drawing }\end{array}$ & Unknown & No Location on Drawing & $\begin{array}{l}\text { Children, Men } \\
\text { at Leisure }\end{array}$ \\
\hline 593 & W1995.030.282 & Untitled & Unknown & $\begin{array}{l}\text { No Date on } \\
\text { Drawing }\end{array}$ & Unknown & No Location on Drawing & $\begin{array}{l}\text { Animals } \\
\text { (Domestic), } \\
\text { Men }\end{array}$ \\
\hline 594 & W1995.030.311 & Untitled & $\begin{array}{l}\text { November } \\
16,1872\end{array}$ & $\begin{array}{c}\text { WVRHC } \\
\text { Description Page }\end{array}$ & Unknown & No Location on Drawing & $\begin{array}{l}\text { Landscape } \\
\text { (Mountains) }\end{array}$ \\
\hline 595 & W1995.030.314 & Untitled & $\begin{array}{l}\text { October } 14, \\
1853\end{array}$ & $\begin{array}{c}\text { Cecil Eby, } \\
\text { "Porte Crayon": } \\
\text { The Life of } \\
\text { David Hunter } \\
\text { Strother, (Chapel } \\
\text { Hill, NC: } \\
\text { University of } \\
\text { North Carolina } \\
\text { Press, 1960), } 78 .\end{array}$ & Weyer's Cave, VA & $\begin{array}{l}\text { WVRHC Description } \\
\text { Page }\end{array}$ & $\begin{array}{l}\text { Landscape } \\
\text { (Caves) }\end{array}$ \\
\hline 596 & W1995.030.323b & Untitled & $\begin{array}{c}\text { October } 3 \\
1873\end{array}$ & $\begin{array}{c}\text { WVRHC } \\
\text { Description Page }\end{array}$ & Unknown & No Location on Drawing & Children \\
\hline 597 & W1995.030.351 & Untitled & June 25,1859 & $\begin{array}{c}\text { WVRHC } \\
\text { Description Page }\end{array}$ & Unknown & No Location on Drawing & $\begin{array}{l}\text { Children, } \\
\text { Women }\end{array}$ \\
\hline 598 & W1995.030.352 & Untitled & Unknown & $\begin{array}{l}\text { No Date on } \\
\text { Drawing }\end{array}$ & Unknown & No Location on Drawing & $\begin{array}{l}\text { Landscape } \\
\text { (Mountains), } \\
\text { Landscape } \\
\text { (Rivers), } \\
\text { Transportation } \\
\text { (Boats) }\end{array}$ \\
\hline
\end{tabular}




\begin{tabular}{|c|c|c|c|c|c|c|c|}
\hline Number & Accession Number & Title & Date & $\begin{array}{l}\text { Source of Date } \\
\text { Information }\end{array}$ & Location & $\begin{array}{l}\text { Source of Location } \\
\text { Information }\end{array}$ & Themes \\
\hline 599 & W1995.030.353 & Untitled & 1859 & $\begin{array}{c}\text { WVRHC } \\
\text { Description Page }\end{array}$ & Italy & $\begin{array}{c}\text { WVRHC Description } \\
\text { Page }\end{array}$ & $\begin{array}{l}\text { Architecture } \\
\text { (Towns), } \\
\text { Children, Men, } \\
\text { Women }\end{array}$ \\
\hline 600 & W1995.030.354 & Untitled & Unknown & $\begin{array}{l}\text { No Date on } \\
\text { Drawing }\end{array}$ & Italy & $\begin{array}{c}\text { WVRHC Description } \\
\text { Page }\end{array}$ & $\begin{array}{l}\text { Architecture } \\
\text { (Towns) }\end{array}$ \\
\hline 601 & W1995.030.361 & Untitled & Unknown & $\begin{array}{l}\text { No Date on } \\
\text { Drawing }\end{array}$ & Unknown & No Location on Drawing & $\begin{array}{l}\text { Decorative } \\
\text { Arts, Men }\end{array}$ \\
\hline 602 & W1995.030.378 & Untitled & Unknown & $\begin{array}{l}\text { No Date on } \\
\text { Drawing }\end{array}$ & Unknown & No Location on Drawing & $\begin{array}{l}\text { Animals } \\
\text { (Domestic), } \\
\text { Men }\end{array}$ \\
\hline 603 & W1995.030.385 & Untitled & $\begin{array}{l}\text { April 16, } \\
1858\end{array}$ & $\begin{array}{c}\text { WVRHC } \\
\text { Description Page }\end{array}$ & Unknown & No Location on Drawing & Men \\
\hline 604 & W1995.030.387pg16b & Untitled & 1874 & $\begin{array}{c}\text { WVRHC } \\
\text { Description Page }\end{array}$ & Unknown & No Location on Drawing & $\begin{array}{l}\text { Men at Work, } \\
\text { Transportation } \\
\text { (Carriages) }\end{array}$ \\
\hline 605 & W1995.030.387pg18 & Untitled & April 5, 1859 & On Drawing & Unknown & No Location on Drawing & $\begin{array}{l}\text { Decorative } \\
\text { Arts, Men }\end{array}$ \\
\hline 606 & W1995.030.387pg27b & Untitled & Unknown & $\begin{array}{l}\text { No Date on } \\
\text { Drawing }\end{array}$ & Unknown & No Location on Drawing & $\begin{array}{l}\text { African } \\
\text { Americans, } \\
\text { Women }\end{array}$ \\
\hline 607 & W1995.030.387pg3 & Untitled & Unknown & $\begin{array}{l}\text { No Date on } \\
\text { Drawing }\end{array}$ & Unknown & No Location on Drawing & $\begin{array}{l}\text { Architecture } \\
\text { (Residential } \\
\text { Buildings), } \\
\text { Landscape } \\
\text { Fences), } \\
\text { Landscape } \\
\text { (Mountains) }\end{array}$ \\
\hline
\end{tabular}




\begin{tabular}{|c|c|c|c|c|c|c|c|}
\hline Number & Accession Number & Title & Date & $\begin{array}{l}\text { Source of Date } \\
\text { Information }\end{array}$ & Location & $\begin{array}{l}\text { Source of Location } \\
\text { Information }\end{array}$ & Themes \\
\hline 608 & W1995.030.387pg38a & Untitled & Unknown & $\begin{array}{l}\text { No Date on } \\
\text { Drawing }\end{array}$ & Unknown & No Location on Drawing & 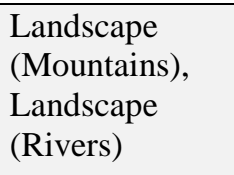 \\
\hline 609 & W1995.030.387pg38b & Untitled & 1845 & $\begin{array}{c}\text { WVRHC } \\
\text { Description Page }\end{array}$ & Unknown & No Location on Drawing & $\begin{array}{l}\text { Animals } \\
\text { (Domestic), } \\
\text { Architecture, } \\
\text { Architecture } \\
\text { (Bridges), } \\
\text { Industry, } \\
\text { Landscape } \\
\text { (Rivers), Men } \\
\text { at Work }\end{array}$ \\
\hline 610 & W1995.030.387pg41 & Untitled & Unknown & $\begin{array}{l}\text { No Date on } \\
\text { Drawing }\end{array}$ & Unknown & No Location on Drawing & Animals (Wild) \\
\hline 611 & W1995.030.387pg42 & Untitled & Unknown & $\begin{array}{l}\text { No Date on } \\
\text { Drawing }\end{array}$ & Unknown & No Location on Drawing & $\begin{array}{l}\text { Landscape, } \\
\text { Men in } \\
\text { Wilderness }\end{array}$ \\
\hline 612 & W1995.030.387pg51 & Untitled & 1845 & $\begin{array}{c}\text { WVRHC } \\
\text { Description Page }\end{array}$ & Unknown & No Location on Drawing & $\begin{array}{l}\text { Animals } \\
\text { (Domestic), } \\
\text { Architecture } \\
\text { (Residential } \\
\text { Buildings), } \\
\text { Landscape } \\
\text { (Fences), } \\
\text { Landscape } \\
\text { (Mountains), } \\
\text { Transportation } \\
\text { (Carriages) }\end{array}$ \\
\hline
\end{tabular}




\begin{tabular}{|c|c|c|c|c|c|c|c|}
\hline Number & Accession Number & Title & Date & $\begin{array}{l}\text { Source of Date } \\
\text { Information }\end{array}$ & Location & $\begin{array}{l}\text { Source of Location } \\
\text { Information }\end{array}$ & Themes \\
\hline 613 & W1995.030.388pg10a & Untitled & Unknown & $\begin{array}{l}\text { No Date on } \\
\text { Drawing }\end{array}$ & Unknown & No Location on Drawing & \\
\hline 614 & W1995.030.388pg10b & Untitled & Unknown & $\begin{array}{l}\text { No Date on } \\
\text { Drawing }\end{array}$ & Unknown & No Location on Drawing & $\begin{array}{l}\text { African } \\
\text { Americans, } \\
\text { Children }\end{array}$ \\
\hline 615 & W1995.030.388pg11 & Untitled & Unknown & $\begin{array}{l}\text { No Date on } \\
\text { Drawing }\end{array}$ & Unknown & No Location on Drawing & $\begin{array}{l}\text { Animals } \\
\text { (Domestic), } \\
\text { Landscape }\end{array}$ \\
\hline 616 & W1995.030.388pg15 & Untitled & July 8,1857 & $\begin{array}{c}\text { WVRHC } \\
\text { Description Page }\end{array}$ & Unknown & No Location on Drawing & Landscape \\
\hline 617 & W1995.030.388pg17 & Untitled & Unknown & $\begin{array}{l}\text { No Date on } \\
\text { Drawing }\end{array}$ & Unknown & No Location on Drawing & Landscape \\
\hline 618 & W1995.030.388pg19a & Untitled & $\begin{array}{l}\text { December 3, } \\
\quad 1856\end{array}$ & $\begin{array}{c}\text { WVRHC } \\
\text { Description Page }\end{array}$ & Unknown & No Location on Drawing & $\begin{array}{l}\text { Decorative } \\
\text { Arts, Men }\end{array}$ \\
\hline 619 & W1995.030.388pg19b & Untitled & Unknown & $\begin{array}{l}\text { No Date on } \\
\text { Drawing }\end{array}$ & Unknown & No Location on Drawing & Men \\
\hline 620 & W1995.030.388pg22 & Untitled & $\begin{array}{l}\text { March 20, } \\
\quad 1856\end{array}$ & $\begin{array}{c}\text { WVRHC } \\
\text { Description Page }\end{array}$ & Unknown & No Location on Drawing & $\begin{array}{l}\text { Children, Men, } \\
\text { Women }\end{array}$ \\
\hline 621 & W1995.030.388pg3 & Untitled & Unknown & $\begin{array}{l}\text { No Date on } \\
\text { Drawing }\end{array}$ & Unknown & No Location on Drawing & $\begin{array}{l}\text { Architecture } \\
\text { (Towns), } \\
\text { Landscape }\end{array}$ \\
\hline 622 & W1995.030.388pg4 & Untitled & Unknown & $\begin{array}{l}\text { No Date on } \\
\text { Drawing }\end{array}$ & Unknown & No Location on Drawing & Children, Men \\
\hline 623 & W1995.030.388pg5 & Untitled & Unknown & $\begin{array}{l}\text { No Date on } \\
\text { Drawing }\end{array}$ & Unknown & No Location on Drawing & $\begin{array}{l}\text { Architecture } \\
\text { (Towns), } \\
\text { Children, Men }\end{array}$ \\
\hline
\end{tabular}




\begin{tabular}{|c|c|c|c|c|c|c|c|}
\hline Number & Accession Number & Title & Date & $\begin{array}{l}\text { Source of Date } \\
\text { Information }\end{array}$ & Location & $\begin{array}{l}\text { Source of Location } \\
\text { Information }\end{array}$ & Themes \\
\hline 624 & W1995.030.388pg6 & Untitled & 1845 & $\begin{array}{c}\text { WVRHC } \\
\text { Description Page }\end{array}$ & Unknown & No Location on Drawing & $\begin{array}{l}\text { Decorative } \\
\text { Arts, Women } \\
\text { at Work }\end{array}$ \\
\hline 625 & W1995.030.388pg8 & Untitled & Unknown & $\begin{array}{l}\text { No Date on } \\
\text { Drawing }\end{array}$ & Unknown & No Location on Drawing & Men \\
\hline 626 & W1995.030.388pg9b & Untitled & Unknown & $\begin{array}{l}\text { No Date on } \\
\text { Drawing }\end{array}$ & Unknown & No Location on Drawing & Children \\
\hline 627 & W1995.030.389pg4 & Untitled & Unknown & $\begin{array}{l}\text { No Date on } \\
\text { Drawing }\end{array}$ & Unknown & No Location on Drawing & Men \\
\hline 628 & W1995.030.389pg5 & Untitled & Unknown & $\begin{array}{l}\text { No Date on } \\
\text { Drawing }\end{array}$ & Unknown & No Location on Drawing & Men \\
\hline 629 & W1995.030.390pg1 & Untitled & Unknown & $\begin{array}{l}\text { No Date on } \\
\text { Drawing }\end{array}$ & Unknown & No Location on Drawing & $\begin{array}{l}\text { Animals } \\
\text { (Domestic), } \\
\text { Transportation } \\
\text { (Carriages) }\end{array}$ \\
\hline 630 & W1995.030.390pg11 & Untitled & Unknown & $\begin{array}{l}\text { No Date on } \\
\text { Drawing }\end{array}$ & Unknown & No Location on Drawing & $\begin{array}{l}\text { Animals } \\
\text { (Domestic), } \\
\text { Transportation } \\
\text { (Carriages) }\end{array}$ \\
\hline 631 & W1995.030.390pg13a & Untitled & Unknown & $\begin{array}{l}\text { No Date on } \\
\text { Drawing }\end{array}$ & Unknown & No Location on Drawing & $\begin{array}{l}\text { African } \\
\text { Americans, } \\
\text { Animals } \\
\text { (Domestic), } \\
\text { Children, Men }\end{array}$ \\
\hline 632 & W1995.030.390pg14a & Untitled & Unknown & $\begin{array}{l}\text { No Date on } \\
\text { Drawing }\end{array}$ & Unknown & No Location on Drawing & $\begin{array}{l}\text { Decorative } \\
\text { Arts, Men at } \\
\text { Leisure, } \\
\text { Women at } \\
\text { Leisure }\end{array}$ \\
\hline
\end{tabular}




\begin{tabular}{|c|c|c|c|c|c|c|c|}
\hline Number & Accession Number & Title & Date & $\begin{array}{l}\text { Source of Date } \\
\text { Information }\end{array}$ & Location & $\begin{array}{l}\text { Source of Location } \\
\text { Information }\end{array}$ & Themes \\
\hline 633 & W1995.030.390pg14b & Untitled & Unknown & $\begin{array}{l}\text { No Date on } \\
\text { Drawing }\end{array}$ & Unknown & No Location on Drawing & $\begin{array}{l}\text { African } \\
\text { Americans, } \\
\text { Children }\end{array}$ \\
\hline 634 & W1995.030.390pg15b & Untitled & Unknown & $\begin{array}{l}\text { No Date on } \\
\text { Drawing }\end{array}$ & Unknown & No Location on Drawing & Men at Work \\
\hline 635 & W1995.030.390pg16a & Untitled & Unknown & $\begin{array}{l}\text { No Date on } \\
\text { Drawing }\end{array}$ & Unknown & No Location on Drawing & $\begin{array}{l}\text { Architecture, } \\
\text { Animals } \\
\text { (Domestic), } \\
\text { Men at Work, } \\
\text { Transportation } \\
\text { (Boats) }\end{array}$ \\
\hline 636 & W1995.030.390pg17 & Untitled & June, 1859 & $\begin{array}{c}\text { WVRHC } \\
\text { Description Page }\end{array}$ & Unknown & No Location on Drawing & $\begin{array}{l}\text { African } \\
\text { Americans, } \\
\text { Children, Men, } \\
\text { Military, } \\
\text { Women }\end{array}$ \\
\hline 637 & W1995.030.390pg19a & Untitled & Unknown & $\begin{array}{l}\text { No Date on } \\
\text { Drawing }\end{array}$ & Unknown & No Location on Drawing & $\begin{array}{l}\text { Transportation } \\
\text { (Carriages), } \\
\text { Women }\end{array}$ \\
\hline 638 & W1995.030.390pg21a & Untitled & Unknown & $\begin{array}{l}\text { No Date on } \\
\text { Drawing }\end{array}$ & Unknown & No Location on Drawing & $\begin{array}{l}\text { Transportation } \\
\text { (Carriages) }\end{array}$ \\
\hline 639 & W1995.030.390pg22 & Untitled & Unknown & $\begin{array}{l}\text { No Date on } \\
\text { Drawing }\end{array}$ & Unknown & No Location on Drawing & $\begin{array}{l}\text { African } \\
\text { Americans, } \\
\text { Men }\end{array}$ \\
\hline 640 & W1995.030.390pg25b & Untitled & $\begin{array}{l}\text { April 22, } \\
1856\end{array}$ & $\begin{array}{c}\text { WVRHC } \\
\text { Description Page }\end{array}$ & Unknown & No Location on Drawing & Children, Men \\
\hline 641 & W1995.030.390pg26 & Untitled & $\begin{array}{c}\text { November } \\
\text { 24, 1858; } \\
\text { December } \\
1858\end{array}$ & $\begin{array}{c}\text { WVRHC } \\
\text { Description Page }\end{array}$ & Unknown & No Location on Drawing & $\begin{array}{l}\text { Animals } \\
\text { (Domestic), } \\
\text { Men, Women }\end{array}$ \\
\hline
\end{tabular}




\begin{tabular}{|c|c|c|c|c|c|c|c|}
\hline Number & Accession Number & Title & Date & $\begin{array}{l}\text { Source of Date } \\
\text { Information }\end{array}$ & Location & $\begin{array}{l}\text { Source of Location } \\
\text { Information }\end{array}$ & Themes \\
\hline 642 & W1995.030.390pg2b & Untitled & Unknown & $\begin{array}{l}\text { No Date on } \\
\text { Drawing }\end{array}$ & Unknown & No Location on Drawing & $\begin{array}{l}\text { Children, } \\
\text { Decorative } \\
\text { Arts, Men at } \\
\text { Leisure, } \\
\text { Women }\end{array}$ \\
\hline 643 & W1995.030.390pg3b & Untitled & April, 1856 & $\begin{array}{c}\text { WVRHC } \\
\text { Description Page }\end{array}$ & Unknown & No Location on Drawing & Men, Women \\
\hline 644 & W1995.030.390pg5b & Untitled & Unknown & $\begin{array}{l}\text { No Date on } \\
\text { Drawing }\end{array}$ & Unknown & No Location on Drawing & Men \\
\hline 645 & W1995.030.390pg6 & Untitled & Unknown & $\begin{array}{l}\text { No Date on } \\
\text { Drawing }\end{array}$ & $\begin{array}{c}\text { Canaan Valley, VA } \\
\text { (WV) }\end{array}$ & $\begin{array}{c}\text { Strother, "The Virginian } \\
\text { Canaan," Harpers New } \\
\text { Monthly Magazine, } \\
\text { December } 1853,27 .\end{array}$ & $\begin{array}{l}\text { Animals } \\
\text { (Domestic), } \\
\text { Landscape } \\
\text { (Mountains), } \\
\text { Men in } \\
\text { Wilderness }\end{array}$ \\
\hline 646 & W1995.030.390pg7 & Untitled & Unknown & $\begin{array}{l}\text { No Date on } \\
\text { Drawing }\end{array}$ & Unknown & No Location on Drawing & $\begin{array}{l}\text { Decorative } \\
\text { Arts }\end{array}$ \\
\hline 647 & W1995.030.390pg9a & Untitled & Unknown & $\begin{array}{l}\text { No Date on } \\
\text { Drawing }\end{array}$ & Unknown & No Location on Drawing & $\begin{array}{l}\text { Children, } \\
\text { Landscape } \\
\text { (Mountains), } \\
\text { Men, Women }\end{array}$ \\
\hline 648 & W1995.030.390pg9b & Untitled & $\begin{array}{l}\text { April 24, } \\
1856\end{array}$ & $\begin{array}{c}\text { WVRHC } \\
\text { Description Page }\end{array}$ & Unknown & No Location on Drawing & Men \\
\hline 649 & W1995.030.391pg10 & Untitled & Unknown & $\begin{array}{l}\text { No Date on } \\
\text { Drawing }\end{array}$ & Unknown & No Location on Drawing & Men \\
\hline 650 & W1995.030.391pg11 & Untitled & 1852 & $\begin{array}{c}\text { WVRHC } \\
\text { Description Page }\end{array}$ & Unknown & No Location on Drawing & $\begin{array}{l}\text { Landscape, } \\
\text { Men }\end{array}$ \\
\hline
\end{tabular}




\begin{tabular}{|c|c|c|c|c|c|c|c|}
\hline Number & Accession Number & Title & Date & $\begin{array}{l}\text { Source of Date } \\
\text { Information }\end{array}$ & Location & $\begin{array}{l}\text { Source of Location } \\
\text { Information }\end{array}$ & Themes \\
\hline 651 & W1995.030.391pg1a & Untitled & Unknown & $\begin{array}{c}\text { No Date on } \\
\text { Drawing }\end{array}$ & Unknown & No Location on Drawing & $\begin{array}{l}\text { Animals } \\
\text { (Domestic), } \\
\text { Men, Women }\end{array}$ \\
\hline 652 & W1995.030.391pg1b & Untitled & Unknown & $\begin{array}{l}\text { No Date on } \\
\text { Drawing }\end{array}$ & Unknown & No Location on Drawing & Landscape \\
\hline 653 & W1995.030.391pg2a & Untitled & Unknown & $\begin{array}{l}\text { No Date on } \\
\text { Drawing }\end{array}$ & Unknown & No Location on Drawing & Children \\
\hline 654 & W1995.030.391pg2b & Untitled & Unknown & $\begin{array}{l}\text { No Date on } \\
\text { Drawing }\end{array}$ & Unknown & No Location on Drawing & $\begin{array}{l}\text { Landscape, } \\
\text { Women }\end{array}$ \\
\hline 655 & W1995.030.391pg3 & Untitled & Unknown & $\begin{array}{l}\text { No Date on } \\
\text { Drawing }\end{array}$ & Unknown & No Location on Drawing & Men, Women \\
\hline 656 & W1995.030.391pg4 & Untitled & Unknown & $\begin{array}{l}\text { No Date on } \\
\text { Drawing }\end{array}$ & Unknown & No Location on Drawing & $\begin{array}{l}\text { Children, Men } \\
\text { Women }\end{array}$ \\
\hline 657 & W1995.030.391pg5a & Untitled & Unknown & $\begin{array}{l}\text { No Date on } \\
\text { Drawing }\end{array}$ & Unknown & No Location on Drawing & Men \\
\hline 658 & W1995.030.391pg5b & Untitled & Unknown & $\begin{array}{l}\text { No Date on } \\
\text { Drawing }\end{array}$ & Unknown & No Location on Drawing & $\begin{array}{l}\text { Landscape, } \\
\text { Men in } \\
\text { Wilderness }\end{array}$ \\
\hline 659 & W1995.030.391pg6 & Untitled & 1852 & $\begin{array}{c}\text { WVRHC } \\
\text { Description Page }\end{array}$ & Unknown & No Location on Drawing & Landscape \\
\hline 660 & W1995.030.391pg7 & Untitled & 1852 & $\begin{array}{c}\text { WVRHC } \\
\text { Description Page }\end{array}$ & Unknown & No Location on Drawing & Men, Women \\
\hline 661 & W1995.030.391pg8b & Untitled & Unknown & $\begin{array}{l}\text { No Date on } \\
\text { Drawing }\end{array}$ & Unknown & No Location on Drawing & $\begin{array}{l}\text { Industry, Men } \\
\text { at Work }\end{array}$ \\
\hline
\end{tabular}




\begin{tabular}{|c|c|c|c|c|c|c|c|}
\hline Number & Accession Number & Title & Date & $\begin{array}{l}\text { Source of Date } \\
\text { Information }\end{array}$ & Location & $\begin{array}{l}\text { Source of Location } \\
\text { Information }\end{array}$ & Themes \\
\hline 662 & W1995.030.392pg3b & Untitled & Unknown & $\begin{array}{l}\text { No Date on } \\
\text { Drawing }\end{array}$ & Unknown & No Location on Drawing & $\begin{array}{l}\text { Animals } \\
\text { (Domestic), } \\
\text { Women }\end{array}$ \\
\hline 663 & W1995.030.392pg9 & Untitled & Unknown & $\begin{array}{l}\text { No Date on } \\
\text { Drawing }\end{array}$ & Unknown & No Location on Drawing & Men \\
\hline 664 & W1995.030.393pg10a & Untitled & Unknown & $\begin{array}{l}\text { No Date on } \\
\text { Drawing }\end{array}$ & Unknown & No Location on Drawing & $\begin{array}{l}\text { African } \\
\text { Americans, } \\
\text { Animals } \\
\text { (Wild), Men }\end{array}$ \\
\hline 665 & W1995.030.393pg14b & Untitled & Unknown & $\begin{array}{l}\text { No Date on } \\
\text { Drawing }\end{array}$ & Unknown & No Location on Drawing & $\begin{array}{l}\text { Men at Leisure, } \\
\text { Men at Work, } \\
\text { Transportation } \\
\text { (Boats), } \\
\text { Women at } \\
\text { Leisure }\end{array}$ \\
\hline 666 & W1995.030.393pg15b & Untitled & $\begin{array}{l}\text { December } 4 \text {, } \\
\quad 1856\end{array}$ & $\begin{array}{c}\text { WVRHC } \\
\text { Description Page }\end{array}$ & Unknown & No Location on Drawing & $\begin{array}{l}\text { Women at } \\
\text { Leisure }\end{array}$ \\
\hline 667 & W1995.030.393pg16a & Untitled & April 8, 1856 & $\begin{array}{c}\text { WVRHC } \\
\text { Description Page }\end{array}$ & Unknown & No Location on Drawing & $\begin{array}{l}\text { Landscape, } \\
\text { Men at Leisure, } \\
\text { Women }\end{array}$ \\
\hline 668 & W1995.030.393pg17a & Untitled & Unknown & $\begin{array}{l}\text { No Date on } \\
\text { Drawing }\end{array}$ & Unknown & No Location on Drawing & $\begin{array}{l}\text { Animals } \\
\text { (Domestic) }\end{array}$ \\
\hline 669 & W1995.030.393pg17b & Untitled & $\begin{array}{l}\text { April 10, } \\
1856\end{array}$ & $\begin{array}{c}\text { WVRHC } \\
\text { Description Page }\end{array}$ & Unknown & No Location on Drawing & $\begin{array}{l}\text { Landscape, } \\
\text { Men in } \\
\text { Wilderness, } \\
\text { Military }\end{array}$ \\
\hline 670 & W1995.030.393pg18a & Untitled & 1852 & $\begin{array}{c}\text { WVRHC } \\
\text { Description Page }\end{array}$ & Unknown & No Location on Drawing & $\begin{array}{l}\text { Animals } \\
\text { (Domestic), } \\
\text { Decorative } \\
\text { Arts }\end{array}$ \\
\hline
\end{tabular}




\begin{tabular}{|c|c|c|c|c|c|c|c|}
\hline Number & Accession Number & Title & Date & $\begin{array}{l}\text { Source of Date } \\
\text { Information }\end{array}$ & Location & $\begin{array}{l}\text { Source of Location } \\
\text { Information }\end{array}$ & Themes \\
\hline 671 & W1995.030.393pg19a & Untitled & Unknown & $\begin{array}{l}\text { No Date on } \\
\text { Drawing }\end{array}$ & Unknown & No Location on Drawing & Men, Women \\
\hline 672 & W1995.030.393pg1b & Untitled & June 5,1859 & $\begin{array}{c}\text { WVRHC } \\
\text { Description Page }\end{array}$ & Unknown & No Location on Drawing & Children \\
\hline 673 & W1995.030.393pg24b & Untitled & $\begin{array}{c}\text { July 23, } \\
\text { 1858; March } \\
13,1859\end{array}$ & $\begin{array}{c}\text { WVRHC } \\
\text { Description Page }\end{array}$ & Unknown & No Location on Drawing & $\begin{array}{l}\text { Animals, } \\
\text { Women }\end{array}$ \\
\hline 674 & W1995.030.393pg27 & Untitled & Unknown & $\begin{array}{l}\text { No Date on } \\
\text { Drawing }\end{array}$ & Unknown & No Location on Drawing & Children \\
\hline 675 & W1995.030.393pg28b & Untitled & Unknown & $\begin{array}{l}\text { No Date on } \\
\text { Drawing }\end{array}$ & Unknown & No Location on Drawing & $\begin{array}{l}\text { Decorative } \\
\text { Arts, Women }\end{array}$ \\
\hline 676 & W1995.030.393pg29a & Untitled & Unknown & $\begin{array}{l}\text { No Date on } \\
\text { Drawing }\end{array}$ & Unknown & No Location on Drawing & $\begin{array}{l}\text { Decorative } \\
\text { Arts }\end{array}$ \\
\hline 677 & W1995.030.393pg29b & Untitled & Unknown & $\begin{array}{l}\text { No Date on } \\
\text { Drawing }\end{array}$ & Unknown & No Location on Drawing & Men, Women \\
\hline 678 & W1995.030.393pg2b & Untitled & Unknown & $\begin{array}{l}\text { No Date on } \\
\text { Drawing }\end{array}$ & Unknown & No Location on Drawing & \\
\hline 679 & W1995.030.393pg30a & Untitled & Unknown & $\begin{array}{l}\text { No Date on } \\
\text { Drawing }\end{array}$ & Unknown & No Location on Drawing & Men \\
\hline 680 & W1995.030.393pg30b & Untitled & 1865 & $\begin{array}{c}\text { WVRHC } \\
\text { Description Page }\end{array}$ & Unknown & No Location on Drawing & Women \\
\hline 681 & W1995.030.393pg31a & Untitled & Unknown & $\begin{array}{l}\text { No Date on } \\
\text { Drawing }\end{array}$ & Unknown & No Location on Drawing & Women \\
\hline
\end{tabular}




\begin{tabular}{|c|c|c|c|c|c|c|c|}
\hline Number & Accession Number & Title & Date & $\begin{array}{l}\text { Source of Date } \\
\text { Information }\end{array}$ & Location & $\begin{array}{l}\text { Source of Location } \\
\text { Information }\end{array}$ & Themes \\
\hline 682 & W1995.030.393pg32 & Untitled & Unknown & $\begin{array}{l}\text { No Date on } \\
\text { Drawing }\end{array}$ & Unknown & No Location on Drawing & $\begin{array}{l}\text { Decorative } \\
\text { Arts }\end{array}$ \\
\hline 683 & W1995.030.393pg35b & Untitled & 1862 & $\begin{array}{c}\text { WVRHC } \\
\text { Description Page }\end{array}$ & Unknown & No Location on Drawing & Men \\
\hline 684 & W1995.030.393pg36a & Untitled & Unknown & $\begin{array}{l}\text { No Date on } \\
\text { Drawing }\end{array}$ & Unknown & No Location on Drawing & Men \\
\hline 685 & W1995.030.393pg5b & Untitled & Unknown & $\begin{array}{l}\text { No Date on } \\
\text { Drawing }\end{array}$ & Unknown & No Location on Drawing & $\begin{array}{l}\text { Animals } \\
\text { (Domestic), } \\
\text { Children }\end{array}$ \\
\hline 686 & W1995.030.393pg6b & Untitled & Unknown & $\begin{array}{l}\text { No Date on } \\
\text { Drawing }\end{array}$ & Unknown & No Location on Drawing & $\begin{array}{l}\text { Architecture, } \\
\text { Men }\end{array}$ \\
\hline 687 & W1995.030.393pg8a & Untitled & Unknown & $\begin{array}{l}\text { No Date on } \\
\text { Drawing }\end{array}$ & Unknown & No Location on Drawing & $\begin{array}{l}\text { African } \\
\text { Americans, } \\
\text { Men at Work, } \\
\text { Transportation } \\
\text { (Boats) }\end{array}$ \\
\hline 688 & W1995.030.394pg14b & Untitled & $\begin{array}{c}\text { November } \\
23,1872\end{array}$ & On Drawing & Unknown & No Location on Drawing & Men \\
\hline 689 & W1995.030.394pg16a & Untitled & Unknown & $\begin{array}{l}\text { No Date on } \\
\text { Drawing }\end{array}$ & Unknown & No Location on Drawing & $\begin{array}{l}\text { Animals } \\
\text { (Domestic), } \\
\text { Landscape } \\
\text { (Mountains), } \\
\text { Men in } \\
\text { Wilderness }\end{array}$ \\
\hline 690 & W1995.030.394pg21a & Untitled & Unknown & $\begin{array}{l}\text { No Date on } \\
\text { Drawing }\end{array}$ & Unknown & No Location on Drawing & $\begin{array}{l}\text { Animals } \\
\text { (Domestic), } \\
\text { Decorative } \\
\text { Arts }\end{array}$ \\
\hline
\end{tabular}




\begin{tabular}{|c|c|c|c|c|c|c|c|}
\hline Number & Accession Number & Title & Date & $\begin{array}{l}\text { Source of Date } \\
\text { Information }\end{array}$ & Location & $\begin{array}{l}\text { Source of Location } \\
\text { Information }\end{array}$ & Themes \\
\hline 691 & W1995.030.394pg23a & Untitled & Unknown & $\begin{array}{l}\text { No Date on } \\
\text { Drawing }\end{array}$ & $\begin{array}{c}\text { Charles Town, VA } \\
\text { (WV) }\end{array}$ & & $\begin{array}{l}\text { Harper's Ferry } \\
\text { Raid Trial, } \\
\text { Men }\end{array}$ \\
\hline 692 & W1995.030.394pg31a & Untitled & Unknown & $\begin{array}{l}\text { No Date on } \\
\text { Drawing }\end{array}$ & $\begin{array}{c}\text { Charles Town, VA } \\
\text { (WV) }\end{array}$ & & $\begin{array}{l}\text { Harper's Ferry } \\
\text { Raid Trial, } \\
\text { Men }\end{array}$ \\
\hline 693 & W1995.030.394pg31b & Untitled & Unknown & $\begin{array}{l}\text { No Date on } \\
\text { Drawing }\end{array}$ & Unknown & No Location on Drawing & $\begin{array}{l}\text { African } \\
\text { Americans, } \\
\text { Men }\end{array}$ \\
\hline 694 & W1995.030.394pg32a & Untitled & Unknown & $\begin{array}{l}\text { No Date on } \\
\text { Drawing }\end{array}$ & Unknown & No Location on Drawing & Men \\
\hline 695 & W1995.030.394pg3b & Untitled & April 2, 1859 & $\begin{array}{c}\text { WVRHC } \\
\text { Description Page }\end{array}$ & Unknown & No Location on Drawing & Men \\
\hline 696 & W1995.030.186b & $\begin{array}{l}\text { Untitled (Man reaching into } \\
\text { his pocket to pay bill) }\end{array}$ & Unknown & $\begin{array}{l}\text { No Date on } \\
\text { Drawing }\end{array}$ & Unknown & No Location on Drawing & Children \\
\hline 697 & W1995.030.190b & $\begin{array}{l}\text { Untitled (Man reaching into } \\
\text { pockets) }\end{array}$ & Unknown & $\begin{array}{l}\text { No Date on } \\
\text { Drawing }\end{array}$ & Unknown & No Location on Drawing & Men, Women \\
\hline 698 & W1995.030.185b & $\begin{array}{l}\text { Untitled (Three Bullies, Boy } \\
\text { With Sword) }\end{array}$ & Unknown & $\begin{array}{l}\text { No Date on } \\
\text { Drawing }\end{array}$ & Unknown & No Location on Drawing & Children \\
\hline 699 & W1995.030.188b & Untitled (Two Faces) & Unknown & $\begin{array}{l}\text { No Date on } \\
\text { Drawing }\end{array}$ & Unknown & No Location on Drawing & Men \\
\hline 700 & W1995.030.395pg14a & Va \& Tenne R.R. July 1874 & $1874 / 1875$ & On Drawing & Unknown & No Location on Drawing & $\begin{array}{l}\text { Men, } \\
\text { Transportation } \\
\text { (Railroads), } \\
\text { Women }\end{array}$ \\
\hline 701 & W1995.030.395pg12 & $\begin{array}{l}\text { Va \& Tenne R.R. July 6th } \\
1874 .\end{array}$ & July 6,1874 & On Drawing & Unknown & No Location on Drawing & $\begin{array}{l}\text { Men, } \\
\text { Transportation } \\
\text { (Railroads), } \\
\text { Women }\end{array}$ \\
\hline
\end{tabular}




\begin{tabular}{|c|c|c|c|c|c|c|c|}
\hline Number & Accession Number & Title & Date & $\begin{array}{l}\text { Source of Date } \\
\text { Information }\end{array}$ & Location & $\begin{array}{l}\text { Source of Location } \\
\text { Information }\end{array}$ & Themes \\
\hline 702 & W1995.030.092 & $\begin{array}{l}\text { Valley of the Arno/ Monte } \\
\text { Heveto Firenze }\end{array}$ & Unknown & $\begin{array}{l}\text { No Date on } \\
\text { Drawing }\end{array}$ & Florence, Italy & On Drawing & $\begin{array}{l}\text { Architecture } \\
\text { (Towns), } \\
\text { Landscape } \\
\text { (Mountains) }\end{array}$ \\
\hline 703 & W1995.030.294 & $\begin{array}{l}\text { Velow Tunnel-Chimneys- } \\
\text { Lawn Gate Natural Tunnel } \\
\text { Scott Co. Va. }\end{array}$ & $\begin{array}{l}\text { November } \\
25,1856\end{array}$ & On Drawing & Scott County, VA & On Drawing & $\begin{array}{l}\text { Landscape } \\
\text { (Mountains) }\end{array}$ \\
\hline 704 & W1995.030.078 & Venice & $\begin{array}{l}\text { April 26, } \\
1843\end{array}$ & On Drawing & Venice, Italy & On Drawing & $\begin{array}{l}\text { Decorative } \\
\text { Arts, Men at } \\
\text { Leisure, } \\
\text { Transportation } \\
\text { (Boats) }\end{array}$ \\
\hline 705 & W1995.030.021 & View from Chimney Cliff & 1852 & $\begin{array}{c}\text { WVRHC } \\
\text { Description Page }\end{array}$ & Unknown & No Location on Drawing & $\begin{array}{l}\text { Landscape } \\
\text { (Mountains) }\end{array}$ \\
\hline 706 & W1995.030.071 & $\begin{array}{l}\text { View from Smoot's hill } \\
\text { overlooking Conrad's Ferry \& } \\
\text { Leesburg }\end{array}$ & June 13,1859 & $\begin{array}{c}\text { WVRHC } \\
\text { Description Page }\end{array}$ & Leesburg, VA & On Drawing & $\begin{array}{l}\text { Architecture, } \\
\text { Landscape }\end{array}$ \\
\hline
\end{tabular}




\begin{tabular}{|c|c|c|c|c|c|c|c|}
\hline Number & Accession Number & Title & Date & $\begin{array}{l}\text { Source of Date } \\
\text { Information }\end{array}$ & Location & $\begin{array}{l}\text { Source of Location } \\
\text { Information }\end{array}$ & Themes \\
\hline 707 & W1995.030.355 & $\begin{array}{l}\text { View from the Hill at Hancock } \\
\text { Md.. Looking toward: Orricks } \\
\text { Hill in Virginia Rebel } \\
\text { Batteries bombarding the } \\
\text { town. December } 1861\end{array}$ & $\begin{array}{c}\text { December } \\
1861\end{array}$ & On Drawing & Hancock, MD & On Drawing & $\begin{array}{l}\text { Architecture } \\
\text { (Institution } \\
\text { Buildings), } \\
\text { Architecture } \\
\text { (Towns), } \\
\text { Landscape } \\
\text { (Mountains), } \\
\text { Men, Military }\end{array}$ \\
\hline 708 & W1995.030.180 & $\begin{array}{l}\text { View of Capstan No. } 3 \text { from } \\
\text { the fish shed }\end{array}$ & Unknown & $\begin{array}{l}\text { Date on Drawing } \\
\text { Unclear }\end{array}$ & Unknown & No Location on Drawing & $\begin{array}{l}\text { Animals } \\
\text { (Wild), } \\
\text { Architecture, } \\
\text { Decorative } \\
\text { Arts, } \\
\text { Landscape } \\
\text { (Mountains), } \\
\text { Landscape } \\
\text { (Lakes), Men } \\
\text { at Work, } \\
\text { Transportation } \\
\text { (Boats) }\end{array}$ \\
\hline 709 & W1995.030.347 & $\begin{array}{l}\text { View of Jefferson College- } \\
\text { Canonsburg Penna. } 1833\end{array}$ & 1833 & On Drawing & Canonsburg, PA & On Drawing & $\begin{array}{l}\text { Architecture } \\
\text { (Institutional } \\
\text { Buildings), } \\
\text { Landscape } \\
\text { (Fences), } \\
\text { Landscape } \\
\text { (Mountains), } \\
\text { Men, Women }\end{array}$ \\
\hline
\end{tabular}




\begin{tabular}{|c|c|c|c|c|c|c|c|}
\hline Number & Accession Number & Title & Date & $\begin{array}{l}\text { Source of Date } \\
\text { Information }\end{array}$ & Location & $\begin{array}{l}\text { Source of Location } \\
\text { Information }\end{array}$ & Themes \\
\hline 710 & W1995.030.360 & $\begin{array}{l}\text { View of the Armoury } \\
\text { buildings \& gap at Harperys } \\
\text { ferry during its occupation by } \\
\text { the Virginia troops May } \\
\text { 23rd..1861 }\end{array}$ & May 23, 1861 & On Drawing & $\begin{array}{l}\text { Harpers Ferry, VA } \\
\text { (WV) }\end{array}$ & On Drawing & $\begin{array}{l}\text { Architecture } \\
\text { (Institutional } \\
\text { Buildings), } \\
\text { Landscape } \\
\text { (Mountains), } \\
\text { Men, Military }\end{array}$ \\
\hline 711 & W1995.030.063 & $\begin{array}{l}\text { View of the Cottage- first } \\
\text { approach }\end{array}$ & 1862 & On Drawing & Unknown & No Location on Drawing & $\begin{array}{l}\text { Animals } \\
\text { (Domestic), } \\
\text { Architecture } \\
\text { (Residential } \\
\text { Buildings), } \\
\text { Landscape } \\
\text { (Fences), } \\
\text { Landscape } \\
\text { (Mountains), } \\
\text { Men }\end{array}$ \\
\hline 712 & W1995.030.322 & $\begin{array}{l}\text { View of the Seymour Estate } \\
\text { Near Moorefield, Hardy Co. } \\
\text { Corn in the Shock }\end{array}$ & June 26,1874 & $\begin{array}{c}\text { WVRHC } \\
\text { Description Page }\end{array}$ & $\begin{array}{l}\text { Hardy County, VA } \\
\text { (WV) }\end{array}$ & On Drawing & $\begin{array}{l}\text { Architecture } \\
\text { (Residential } \\
\text { Buildings), } \\
\text { Landscape } \\
\text { (Mountains) }\end{array}$ \\
\hline 713 & W1995.030.065 & $\begin{array}{l}\text { View of the Spring House at } \\
\text { Brown's Cottage Brownsville, } \\
\text { Washington Co. }\end{array}$ & 1862 & On Drawing & Brownsville, MD & On Drawing & $\begin{array}{l}\text { Architecture } \\
\text { (Residential } \\
\text { Buildings), } \\
\text { Landscape, } \\
\text { Men at Leisure }\end{array}$ \\
\hline 714 & W1995.030.297 & $\begin{array}{l}\text { View of the University of } \\
\text { Va..taken Nov: } 1853 \text { (En } \\
\text { verso: Within a view of the } \\
\text { University of Va.) }\end{array}$ & $\begin{array}{c}\text { November } 2 \\
1853\end{array}$ & $\begin{array}{c}\text { WVRHC } \\
\text { Description Page }\end{array}$ & Charlottesville, VA & On Drawing & Men \\
\hline
\end{tabular}




\begin{tabular}{|c|c|c|c|c|c|c|c|}
\hline Number & Accession Number & Title & Date & $\begin{array}{l}\text { Source of Date } \\
\text { Information }\end{array}$ & Location & $\begin{array}{l}\text { Source of Location } \\
\text { Information }\end{array}$ & Themes \\
\hline 715 & W1995.030.387pg9 & $\begin{array}{l}\text { Warm Spring Ridge } \\
\text { September } 221846\end{array}$ & $\begin{array}{l}\text { September } \\
22,1846\end{array}$ & On Drawing & $\begin{array}{c}\text { Warm Springs Ridge, } \\
\text { VA (WV) }\end{array}$ & On Drawing & Landscape \\
\hline 716 & W1995.030.387pg8 & Warm Springs Ridge 1845 & 1845 & On Drawing & Unknown & No Location on Drawing & Landscape \\
\hline 717 & W1995.030.247 & $\begin{array}{l}\text { Wash. Higgins Well I've a } \\
\text { mind to see how I look Take } \\
\text { my picter Dec 9th } 1856\end{array}$ & $\begin{array}{c}\text { December } 9 \\
1856\end{array}$ & On Drawing & Unknown & No Location on Drawing & Men \\
\hline 718 & W1995.030.306 & $\begin{array}{l}\text { Washington's Hall. Weyer's } \\
\text { cave. Octo 15th..1853 }\end{array}$ & $\begin{array}{l}\text { October 14, } \\
1853\end{array}$ & On Drawing & Weyer's Cave, VA & On Drawing & $\begin{array}{l}\text { Landscape } \\
\text { (Caves) }\end{array}$ \\
\hline 719 & W1995.030.206a & $\begin{array}{l}\text { Well General I reckon you } \\
\text { know Swartz. May } 5,1862 . \\
\text { Strasburg }\end{array}$ & May 15,1862 & On Drawing & Strasburg, VA & On Drawing & Men \\
\hline 720 & W1995.030.394pg19 & $\begin{array}{l}\text { Whalers just landed in New } \\
\text { Bedford }\end{array}$ & May 20, 1859 & On Drawing & New Bedford, MA & On Drawing & $\begin{array}{l}\text { Animals } \\
\text { (Domestic), } \\
\text { Men at Leisure, } \\
\text { Men at Work, } \\
\text { Transportation } \\
\text { (Carriages) }\end{array}$ \\
\hline 721 & W1995.030.343 & $\begin{array}{l}\text { White Sulphur Springs Oct } \\
\text { 26th..- }\end{array}$ & $\begin{array}{l}\text { October 26, } \\
\text { Unknown } \\
\text { Year }\end{array}$ & On Drawing & $\begin{array}{c}\text { White Sulphur Springs, } \\
\text { VA (WV) }\end{array}$ & On Drawing & $\begin{array}{l}\text { Architecture } \\
\text { (Institutional } \\
\text { Buildings), } \\
\text { Landscape } \\
\text { (Mountains) }\end{array}$ \\
\hline 722 & W1995.030.395pg15 & $\begin{array}{l}\text { Windows - Castle Rocks. } \\
\text { Kanawha - May 11th } 1872 .\end{array}$ & May 11,1872 & On Drawing & $\begin{array}{c}\text { Kanawha County, VA } \\
\text { (WV) }\end{array}$ & On Drawing & $\begin{array}{l}\text { Landscape } \\
\text { (Mountains) }\end{array}$ \\
\hline 723 & W1995.030.387pg6b & Winter of 1853 Norborne Hall & 1853 & On Drawing & Martinsburg, VA (WV) & $\begin{array}{l}\text { Cecil Eby, "Porte } \\
\text { Crayon": The Life of } \\
\text { David Hunter Strother, } \\
\text { (Chapel Hill, NC: } \\
\text { University of North } \\
\text { Carolina Press, 1960), } 62 .\end{array}$ & $\begin{array}{l}\text { Decorative } \\
\text { Arts, Women }\end{array}$ \\
\hline
\end{tabular}




\begin{tabular}{|c|c|c|c|c|c|c|c|}
\hline Number & Accession Number & Title & Date & $\begin{array}{l}\text { Source of Date } \\
\text { Information }\end{array}$ & Location & $\begin{array}{l}\text { Source of Location } \\
\text { Information }\end{array}$ & Themes \\
\hline 724 & W1995.030.236 & $\begin{array}{l}\text { Wm. Henderson. Stensley. } \\
\text { Dec. 9th..1856. }\end{array}$ & $\begin{array}{c}\text { December 9, } \\
1856\end{array}$ & On Drawing & Unknown & No Location on Drawing & Men \\
\hline 725 & W1995.030.299 & $\begin{array}{l}\text { Woodstock. From the Rail } \\
\text { Road above the town } \\
\text { Shenandoah Co: Virginia } \\
\text { April 14th } 1862 \text {. }\end{array}$ & $\begin{array}{l}\text { April 14, } \\
1862\end{array}$ & On Drawing & Woodstock, VA & On Drawing & $\begin{array}{l}\text { Architecture } \\
\text { (Towns), } \\
\text { Landscape, } \\
\text { Transportation } \\
\text { (Railroads) }\end{array}$ \\
\hline 726 & W1995.030.382a & $\begin{array}{l}\text { X. hastens to get a shot at a } \\
\text { deer }\end{array}$ & Unknown & $\begin{array}{l}\text { No Date on } \\
\text { Drawing }\end{array}$ & $\begin{array}{c}\text { Canaan Valley, VA } \\
\text { (WV) }\end{array}$ & $\begin{array}{l}\text { Strother, "The Virginian } \\
\text { Canaan," Harpers New } \\
\text { Monthly Magazine, } \\
\text { December } 1853,31 .\end{array}$ & $\begin{array}{l}\text { Men in } \\
\text { Wilderness }\end{array}$ \\
\hline 727 & W1995.030.321 & $\begin{array}{l}\text { Yellow Banks View at } \\
\text { Romney-Hampshire Co. West } \\
\text { Va.. }\end{array}$ & $\begin{array}{l}\text { November } \\
27,1874\end{array}$ & $\begin{array}{c}\text { WVRHC } \\
\text { Description Page }\end{array}$ & Romney, VA (WV) & On Drawing & $\begin{array}{l}\text { Landscape } \\
\text { (Mountains) }\end{array}$ \\
\hline 728 & W1995.030.393pg33a & Young Alabama. Kingston. & Unknown & $\begin{array}{l}\text { No Date on } \\
\text { Drawing }\end{array}$ & Kingston, Alabama & On Drawing & $\begin{array}{l}\text { African } \\
\text { Americans, } \\
\text { Animals } \\
\text { (Domestic), } \\
\text { Men, Women }\end{array}$ \\
\hline 729 & W1995.030.298 & $\begin{array}{l}\text { Young Guard of Richond Va. } \\
\text { protecting the old Church } \\
\text { Tower of Jamestown Island }\end{array}$ & May 1857 & On Drawing & Jamestown, VA & On Drawing & $\begin{array}{l}\text { Architecture, } \\
\text { Men, Military }\end{array}$ \\
\hline 730 & W1995.030.394pg6 & $\begin{array}{l}\text { Young Shark } 3 \text { feet } 6 \text { inches } \\
\text { long/ Pigfish - a small fish that } \\
\text { grunts like a pig }\end{array}$ & May 28, 1859 & On Drawing & Unknown & No Location on Drawing & Animals (Wild) \\
\hline
\end{tabular}

